1 A dynamic, spatially periodic, micro-pattern of HES5 underlies neurogenesis in \title{
the mouse spinal cord.
}

Biga $\mathrm{V}^{1 *}$, Hawley $\mathrm{J}^{1 *}$, Soto, $\mathrm{X}^{1}$, Johns $\mathrm{E}^{1}$, Han $\mathrm{D}^{2}$, Bennett $\mathrm{H}^{1}$, Adamson $A, \mathrm{D}^{1}$ Kursawe, $\mathrm{J}^{3}$, Glendinning $\mathrm{P}^{2}$, Manning C. $\mathrm{S}^{1 *} \#$. and Papalopulu N\#

[1] Faculty of Biology Medicine and Health, The University of Manchester, Oxford Road, Manchester, M13 9PL, UK

[2] Department of Mathematics, School of Natural Sciences, Faculty of Science and

Engineering, The University of Manchester, Oxford Road, Manchester, M13 9PL, UK

[3] School of Mathematics and Statistics, University of St Andrews, St Andrews, KY16 9SS, UK.

\# Corresponding authors, ${ }^{*}$ These authors have contributed equally to this work.

VB, JH and CSM contributed equally.

CSM and NP contributed equally.

Running title: HES5 micro-patterns explain rate of neurogenesis

\section{Abstract}

Ultradian oscillations of HES Transcription Factors (TFs) at the single cell level, enable cell state transitions. However, the tissue level organisation of HES5 dynamics in neurogenesis is unknown. Here, we analyse the expression of HES5 exvivo in the developing mouse ventral spinal cord and identify microclusters of 4-6 cells with positively correlated HES5 level and ultradian dynamics. These microclusters are spatially periodic along the dorsoventral axis and temporally dynamic, alternating between high and low expression with a supra-ultradian persistence time. We show that Notch signaling is required for temporal dynamics but not the spatial periodicity of HES5. Few Neurogenin-2 cells are observed per cluster, irrespective of high or low state, suggesting that the microcluster organization of HES5 enables the stable selection of differentiating cells. Computational modelling predicts that different cell coupling strengths underlie the HES5 spatial patterns and rate of differentiation, which is consistent with comparison between the motoneuron and interneuron progenitor domains. Our work shows a previously unrecognised spatiotemporal organisation of neurogenesis, emergent at the tissue level from the synthesis of single cell dynamics. 
35 Keywords (5): Neurogenesis, HES5, gene expression, oscillations, developmental

36 patterning

37 Synopsis

38 Live imaging of HES5 expression in the ventral mouse spinal cord together with

39 computational modelling is used to identify and analyse spatially periodic HES5

40 micro-patterns that emerge from the synthesis of single cell dynamics.

- HES5 is expressed in spatially periodic microclusters along the dorsalventral axis in spinal cord that are dynamically maintained by Notch signalling.

- Microclusters can arise, in part, from single cell oscillators that are synchronous and weakly coupled via Notch.

- Spatial patterns are different between motorneuron and interneuron progenitor domains and the probability for progenitor differentiation is regulated by the coupling strength between cells.

- NGN2 is also spatially periodic along the dorso-ventral axis and microclusters of HES5 may act to pick a single NGN2 high cell for differentiation. 


\section{Introduction}

Neurogenesis is the developmental process which generates the variety of neuronal cell types that mediate the function of the nervous system. Neurogenesis takes place over a period of days during mouse embryogenesis, thus, the transition from progenitor maintenance to differentiation needs to be balanced for development to occur normally. Neurogenesis relies on the integration of positional information with the transcriptional programme of neuronal differentiation. In the spinal cord, notable progress has been made in understanding the role and regulation of the dorsoventral (D-V) positional system, that relies on secreted morphogens and transcriptional networks to generate the stereotyped array of different types of neurons along this axis (Briscoe \& Small, 2015; Sagner \& Briscoe, 2019). The transcriptional programme that mediates neurogenesis is also well understood in the spinal cord, particularly with the application of single cell sequencing (Delile et al, 2019; Paridaen \& Huttner, 2014; Sagner \& Briscoe, 2019).

Recent live imaging studies of cell fate decisions during neurogenesis have added a new dimension to this knowledge (Das \& Storey, 2012, 2014; Manning et al, 2019a; Nelson et al, 2020; Soto et al, 2020; Vilas-Boas et al, 2011). They have shown the importance of understanding transcription factor (TF) expression dynamics in real time, including the key transcriptional basic helix-loop-helix repressors Hairy and enhancer of split (HES)1 and 5 (Bansod et al, 2017; Imayoshi \& Kageyama, 2014; Ohtsuka et al, 1999), in regulating state transitions. We have previously shown that in spinal cord tissue, HES5 exhibits ultradian periodicity of $3-4 \mathrm{~h}$ in about half of the progenitor population with the remaining progenitors showing aperiodic fluctuations (Manning et al., 2019a). The percentage of cells that show oscillations rises in cells that enter the differentiation pathway; such cells show a transient phase of more coherent oscillations before the level of HES5 is downregulated in differentiated cells (Manning et al., 2019a). Furthermore, our studies of a zebrafish paralogue Her6 showed that the transition from aperiodic to oscillatory expression is needed for neuronal differentiation, suggesting that oscillatory expression has an enabling role for cell state transitions (Soto et al., 2020) as we have previously predicted computationally (Bonev et al, 2012; Goodfellow et al, 2014; Phillips et al, 2016).

Although these studies revealed an unappreciated dynamic behaviour at the level of HES TF protein expression, these live imaging studies are based on recording dynamics from sparsely distributed single cells in the tissue context. Therefore, little 
102 is known about how single cell dynamics are synthesised to tissue level dynamics.

103 Do cells interact with their neighbours in order to coordinate their cell state

104 transitions and if so, how and what is the mechanism?

105 Notch is of particular interest in this context because it is a highly conserved cell to

106 cell signalling pathway that is well known for generating complex spatial patterns of

107 cell fates in tissue development (Cohen et al, 2010; Corson et al, 2017; Henrique \&

108 Schweisguth, 2019; Hunter et al, 2016; Shaya \& Sprinzak, 2011). Activation of Notch

109 receptors by Notch ligands, including DLL1 and JAG1, results in downstream

110 expression of HES1 and HES5. HES TFs can influence Notch activity on

111 neighbouring cells by repressing Notch ligand expression either directly (de

112 Lichtenberg et al, 2018; Kobayashi et al, 2009) or indirectly through the repression of

113 proneural TFs such as Neurogenin1/2 (NGN1/2) (Ma et al, 1998). We argue that in

114 order to understand how the balance of progenitor factor HES can be tipped in

115 favour of a proneural factor giving rise to a decision point in neural progenitor cells,

116 we need to address tissue-level patterns of HES expression and use computational

117 models that can integrate the complexity of interactions at multiple scales.

118 The effects of Notch-Delta signaling combined with HES oscillations have been

119 investigated during somitogenesis. Live imaging of dissociated PSM cells in vitro has

120 shown that single cell oscillators can self-organise through Notch dependent

121 synchronisation to generate waves in gene expression similar to those observed in

122 vivo (Tsiairis \& Aulehla, 2016). A model of mRNA and protein production and self-

123 repression with transcriptional delay explains the emergence of autonomous

124 oscillations of Her1 and Her7 as well as synchronization by Notch activity observed

125 during the formation of somites (Lewis, 2003; Özbudak \& Lewis, 2008; Webb et al,

126 2016). A more abstract Kuramoto-style model with time delays explains how a

127 population of initially asynchronous and autonomous oscillators can evolve to adopt

128 the same frequency and phase in order to periodically form somites (Morelli et al,

129 2009; Oates, 2020). The period of the oscillations determines the size of the somite

130 and Notch abundance controls dynamic parameters such as the time to

131 synchronisation (Herrgen et al, 2010). Apart from a limited number of studies

132 suggesting an anti-phase relationship of DLL1 oscillations in neighbouring cells in

133 neural cells (Shimojo et al, 2016), whether and how neural progenitor cells co-

134 ordinate fate decisions and dynamic HES activity with their neighbours remains 135 unknown. 
136 In this study, we observe spatially periodic HES5 micro-patterns that can be

137 generated by local synchronisation of low coherence single cell oscillators present in

138 spinal cord tissue and these patterns are maintained in a dynamic way through

139 Notch mediated cell-cell interactions. A computational model predicts that coupling

140 strength changes spatial patterns of expression and in turn, the probability of

141 progenitor differentiation. We confirm that between adjacent progenitor domains in

142 the spinal cord, the rate of differentiation correlates with spatial patterns of HES5 and

143 cell-cell coupling strength. Thus, organisation of neural progenitors in HES5 phase-

144 synchronised and level-matched progenitors, is an exquisite spatiotemporal

145 mechanism conferring tissue level regulation of the transition of single cells from

146 neural progenitor to neuron.

148 Results

149 Positive correlations in Venus::HES5 intensity are indicative of microclusters

150 in spinal cord tissue

151 Within the peak of spinal cord neurogenesis (E9.5-E11.5), HES5 is expressed in two

152 broad domains in the dorsal and ventral embryonic mouse spinal cord (Manning et 153 al., 2019a; Sagner et al, 2018). Previously we have characterised the single cell

154 dynamic behaviour of the more ventral HES5 expression domain that covers the 155 ventral interneuron (p0/p2) and motorneuron progenitors (p2/pMN) (Manning et al., 156 2019a). Thus, to understand how the single cell expression dynamics contributes to 157 tissue level behaviour we have focused here on the same ventral area of HES5 158 expression (Figure 1 and Figure EV1a). In this area, all progenitor cells (marked by 159 SOX2) show HES5 expression (Figure EV1b). To characterise the spatial pattern of 160 HES5 protein expression in this progenitor domain we made ex-vivo slices of E9.5161 E11.5 Venus::HES5 knock-in mouse embryo spinal cord (Imayoshi et al, 2013). In 162 snapshot images of this domain we noticed multiple local clusters of neural 163 progenitor cells with similar levels of nuclear HES5 (Figure 1a) which we refer to as 164 'microclusters'. These are notable after manual segmentation using a Draq5 live 165 nuclear stain and averaging HES5 intensity across the nucleus (Figure 1a-c). The 166 differences in Venus::HES5 intensity between nuclei did not correlate with the Draq5 167 nuclear staining indicating this was not related to global effects or effects of imaging 168 through tissue (Figure EV1c). By measuring the number of nuclei in microclusters 169 with high Venus::HES5 levels we found that they consisted of 3-4 cells wide in the 
170 apical-basal (A-B) and 2-4 cells wide in the dorso-ventral (D-V) direction (3-7 cells in 171 total) and were similar in size between E9.5 and E11.5 (Figure 1d). Consistent with

172 the presence of microclusters of cells with similar levels, nuclei showed a positive 173 correlation in Venus::HES5 between neighbours 1 to 4 (Figure 1e). We took a more 174 quantitative approach and correlated mean nuclear HES5 levels between all pairs of 175 nuclei and found that nuclei close to each other were highly positively correlated and 176 this correlation dropped with increasing distance, becoming negative at distances 177 over $50 \mu \mathrm{m}$ (Figure 1f). This relationship was similar across E9.5-E11.5 (Figure 178 EV1d) and substantially different to the correlation coefficients calculated from 179 randomisations of the nuclei intensities but keeping the same nuclear spatial 180 arrangement (Figure 1f) which indicates the presence of a pattern in HES5 levels.

181 The longer-range negative correlations may arise from gradients in HES5 expression 182 in $A-B$ and $D-V$ direction. Indeed the images indicate the presence of a radial 183 gradient emanating from an area of highly expressing cells (Figure 1g,h and Figure 184 EV1e,f). Such a radial gradient could be due to the downregulation of HES5 as cells 185 differentiate and move basally from the progenitor domain as well as to D-V 186 differences in the level of expression (see below), and is not further investigated 187 here. To ask whether the local positive correlations in HES5 levels are an artefact of 188 this larger-scale domain expression pattern, we measured and subsequently 189 removed a radial gradient across the tissue from the segmented single cell images 190 (see Materials and Methods). However even after removing a radial gradient, mean 191 nuclear HES5 levels at E9.5-E11.5 remained highly positively correlated at distances 192 less than 40-50 $\mu \mathrm{ms}$ (Figure 1i and. Figure EV1g,h). Therefore, a global tissue 193 gradient of HES5 cannot fully explain the detailed spatial pattern and further factors, 194 such as microclusters of cells with similar HES5 levels, must contribute to the 195 formation of the HES5 spatial pattern.

197 HES5 microclusters are spatially periodic along dorso-ventral axis of spinal 198 cord

199 The high-resolution analysis of single-cell snapshots showed the presence of 200 multiple microclusters in HES5 expression in the ventral domain. Next, we asked 201 whether these microclusters have a regular spatial arrangement. To do this we drew 202 line profiles $15 \mu \mathrm{m}$ wide, parallel to the ventricle, in the ventral to dorsal direction 
203 (Figure 2a and Figure EV2a,b) and plotted the Venus::HES5 intensity along this line

204 (Figure 2b) from lower resolution 20x images of ex-vivo slice cultures. Throughout

205 the paper the 0 distance is the ventral most point of the HES5 domain, and distance

206 extends dorsally (Materials and Methods). Detrending the signal removed a bell-

207 shaped curve of expression that is a result of different HES5 levels along the D-V

208 axis and is not further investigated here (Figure $\mathbf{2 b}$ and Figure EV2c). The

209 microclusters could be detected as peaks in the detrended Venus::HES5 intensity

210 profile (direct comparison in Figure EV2a-c) and we observed multiple peaks across

211 the D-V axis (Figure 2c). Periodicity analysis of the detrended spatial Venus::HES5

212 expression profile revealed the presence of significant peaks in the power spectrum

213 (Figure 2d) and multiple significant peaks in the auto-correlation function in all

214 tissues analysed (Figure 2e, Appendix Figure S1a and Appendix Table S1). A

215 significant peak in the auto-correlation function shows the signal has similarity to

216 itself at the lag indicated in the x-axis. Multiple peaks in an autocorrelation function

217 are indicative of a periodic signal with the peak-to-peak distance in the

218 autocorrelation corresponding to the period of the signal. The spatial period in

219 Venus::HES5 expression was $30 \mu \mathrm{m}$ to $60 \mu \mathrm{m}$ with a median period of $40 \mu \mathrm{m}$ and no

220 significant difference between E9.5 to E11.5. Periodicity measurements from auto-

221 correlation functions and the power spectrum corresponded well (Figure $2 f$ and

222 Appendix Figure S1b).

223 To understand how our observed spatial periodicity relates to nuclear count in the

224 tissue, we analysed the spatial profile for the Draq5 nuclear stain from snapshot

225 images of Venus::HES5 ex-vivo slices. We observed peaks in Draq5 in regions of

226 low Venus::HES5 indicating that the lower Venus::HES5 regions did not correspond

227 to a lack of nuclei at this position of the tissue (Figure EV2d). As expected, the

228 Draq5 signal observed along the D-V axis also showed multiple significant peaks in

229 the autocorrelation that corresponded to a spatial period of $10 \mu \mathrm{m}$, a single-cell width

230 and was consistent over developmental time (Figure 2g,h, Figure EV2e and

231 Appendix Figure S1c). Using this value we estimate that the periodic occurrence of

232 microclusters of cells with correlated levels of Venus::HES5 has a median period of 4

233 cells.

234 Since the apical region of spinal cord contains proliferative neural progenitors with

235 high levels of HES5 that become downregulated when progenitors begin to migrate

236 towards basal regions, we interrogated if the spatial periodicity persisted in the A-B 
237 axis. Venus::HES5 expression profiles collected from apical, intermediate and basal

238 regions (Figure 2i) within the HES5 expression domain at E10.5 all showed spatial

239 periodicity (both power spectrum and auto-correlation, Figure EV2f) with the period

240 varying from approximately 4 cells in the apical side to 3 cells in the basal region

241 (Figure 2j, Appendix Figure S1d). These results suggest that proliferative

242 progenitors (localised apically) as well as differentiating progenitors (localised more

243 basally) show local spatial correlation in Venus::HES5 levels between neighbouring

244 cells where 3-7 neighbouring cells can be in a high or low state in synchrony with

245 each other and that these clusters are repeated periodically in the D-V axis.

246 To test whether clusters extended in the anterior-posterior (A-P) axis we took

247 longitudinal cryo-sections of the spinal cord and performed auto-correlations of the

248 Venus::HES5 spatial profile along the A-P axis (Figure EV2g,h). Peaks in the auto-

249 correlation show spatial periodicity in A-P axis of around 30um (Figure EV2i). Thus,

250 the scale of cluster size in A-P is comparable to that observed in D-V. We confirmed

251 this in our existing kymograph data by correlating the expression of HES5 at

252 subsequent z-positions extending in the A-P axis in the same slice (Materials and

253 Methods). Indeed, correlations in A-P persisted at less than 30um but were lost

254 further away (Figure EV2j).

255 The microclusters could be set up earlier on in development with fewer or single 256 cells, and then clonally expand through cell division. However the similar

257 microcluster size and Venus::HES5 spatial periodicity between E9.5, E10.5, and

258 E11.5 argues against a clonal expansion mechanism. Co-ordinated cell behaviours

259 such as nuclear-motility may also contribute. We found weak positive correlation in

260 the movement of nuclei in apico-basal axis between cell pairs less than 30 $\mu \mathrm{m}$ apart,

261 but there was a large variation in correlations, and the correlation dropped between

262 cells further apart (Appendix Figure S1e). This weak correlation in apical-basal

263 nuclear movement may contribute weakly to maintaining microcluster pattern.

265 The HES5 spatial pattern is dynamic over time

266 We next investigated whether the spatially periodic pattern in Venus::HES5 is also

267 dynamic over time. To do this, we generated kymographs, single images that 268 represent spatial intensity profiles in the same region of tissue over time, from $15 \mu \mathrm{m}$ 269 wide ventral-dorsal lines in movies of E10.5 Venus::HES5 spinal cord ex vivo slices 
270 (Figure 3a,b, Appendix Figure S2a,b and Appendix Movie S1). We noticed

271 stripes in the kymograph, corresponding to the spatially periodic Venus::HES5

272 pattern (Figure 3b). To investigate how long high HES5 and low HES5 clusters

273 persist over time we split the kymograph into adjacent $20 \mu \mathrm{m}$ regions (half of the

$27440 \mu \mathrm{m}$ spatial periodicity, chosen to capture the size of a microcluster) along the D-V

275 axis and followed their levels over time (Methods). Hierarchical clustering of the

276 dynamic behaviour of the kymograph regions revealed changes from low to high

277 Venus::HES5, high to low, or re-occurring high-low-high, showing that clusters of

278 cells can interconvert between low and high HES5 states (Figure 3c and additional

279 examples Appendix Figure S2c). To exclude the possibility of sample drift in the DV

280 axis being responsible for these dynamics, we used single cell tracking from the

281 same videos as the kymographs to determine global DV drift is minimal (<20um per

282 12h, Appendix Figure S2) and only one in 10 tissues was excluded from temporal

283 analysis. Thus we could proceed to analyse the persistence of a cluster in the high

284 or low state and we found that it was on average 6-8 hours with no significant

285 difference between persistence of high or low states in the same region (Figure

$2863 \mathbf{3 d}, \mathbf{e})$. We confirmed these results using a second method that detected high/low

287 regions in the first 2 hrs of kymograph and fixing ROls around these regions whereby

288 we continued to observe changes in intensity over time (Appendix Figure S2d,e).

289 This shows that the micro-stripes of HES5 expression are not stable but are dynamic

290 over time.

291 Since the HES5 expression is periodic along the D-V axis, it can be represented as a

292 spatial oscillator. Therefore, we used its phase characteristics denoting the position

293 in the spatial cycle, to analyse how the HES5 signal changes from high to low in the

294 same region over time. We transformed the detrended spatial Venus::HES5 intensity

295 (Figure EV2k, Appendix Figure S3a) along the D-V axis to phase of the spatial

296 oscillator of Venus::HES5 using the Hilbert transform (Methods). All experiments

297 showed a dynamic pattern with changes in phase in any area of the tissue over the

29812 to $24 \mathrm{hr}$ movies (Appendix Figure S3b). Regions could be identified that

299 maintained a similar phase over several hours followed by a change, indicating a

300 switch in state of the Venus::HES5 pattern (Figure EV2k). Phase waves could be

301 observed in some movies, indicated by the diagonal lines of similar colours in the

302 spatial phase map (Appendix Fig S3biii), however these were variable across the 
303 data and did not have a consistent direction in the D-V axis between experiments. In

304 summary, we find microclusters of cells with correlated Venus::HES5 levels that are

305 a maximum of 2-3 cells wide in D-V and 3-4 in A-B axes and are arranged in a 306 spatially periodic pattern. The pattern is also temporally dynamic with a persistence 307 in a high or low level expression of 6-8 hrs but no consistent phase wave travel in D$308 \mathrm{~V}$.

310 Correlations in Venus::HES5 levels over time and local co-ordination in phase 311 is observed in single cells in tissue

312 We next addressed how the dynamic tissue pattern relates to single cell behaviour 313 and Venus::HES5 expression. We previously tracked sparse single nuclei labelled 314 with inducible Cre-mediated H2B::mCherry in E10.5 Venus::HES5 spinal cord ex 315 vivo slices and reported that about half of the progenitors show oscillations of period 316 3-4 hrs, while others show aperiodic fluctuations (Manning et al, 2019b). However, 317 the persistence of HES5 high or low microclusters in the spatial pattern is around $2 x$ 318 the period of Venus::HES5 temporal oscillations. This supra-ultradian behaviour that 319 emerged from the tissue level analysis has not been reported before but we 320 hypothesised that it may correspond to "longer-term" trends in the Venus::HES5 321 mean level (Appendix Figure S4a). These have been previously observed at the 322 single cell level, modulating the mean level of the ultradian oscillations/fluctuations, 323 but not analysed further. Thus, we re-analysed single cell Venus::HES5 expression 324 data using the same methods to analyse persistence and found similar values in 325 persistence time between microclusters and single cell long-term Venus::HES5 326 trends (Figure 3f). This suggests that changes in the levels of Venus::HES5 in 327 microclusters are produced at single cell level through changes in the HES5 trend 328 indicative of mean abundance levels.

329 We next identified single cell pairs that maintained close proximity $(<20 \mu \mathrm{m})$ over 12

330 hrs (Figure $\mathbf{3 g}, \mathbf{h}$ ) and we refer to these as cell pairs. We found that $13 / 14$ cell pairs 331 showed a high positive correlation (Figure 3j: raw $\geq 0.5$, median 0.88 and examples 332 Figure 3i) in their mean Venus::HES5 levels which persisted in over $70 \%$ of pairs 333 after correction to mean tissue fluorescence to account for photobleaching (Figure 334 3j: rel. to tissue $\geq 0.5$, median 0.74 and examples Figure $3 \mathbf{i}$ insert). The experimental 335 control of nuclear H2B:mCherry in the same cells showed no strong correlation 336 (Figure $\mathbf{3 i}$, control <0.5, median -0.2). Furthermore, the cell pairs persistently 
337 showed in-phase Venus::HES5 peaks (examples Figure 3k red arrowhead) and

338 phase visualisation maps of all pairs exhibited a large accumulation of Venus::HES5

339 instantaneous phases along the diagonal between $(0,2 \pi)$ and $(2 \pi, 2 \pi)$ indicating

340 prevalence of local in-phase behaviour at single cell level (Figure 3I). We also noted

341 phase activity at $(0,2 \pi)$ corresponding to anti-phase peaks, so we interrogated this

342 further using cross-correlation analysis of cell pairs (Appendix Figure S4b-d) which

343 indicated that the large majority of pairs show only minimal phase shift between them

344 (Appendix Figure S4d, median 15 min coinciding with sampling rate). These

345 findings demonstrate that ultradian activity between neighbouring cell pairs in spinal

346 cord tissue is predominantly in-phase and we refer to this as 'local in-phase'.

347 To summarise, inside a microcluster cells predominantly show synchronised

348 ultradian oscillations of 3-4 hrs and each microcluster has a persistence time in a

349 high or low state of about 6-8hrs coinciding with the persistence of single cell trends.

350 A microcluster is a composite of these two activities.

351

352 Notch inhibition extinguishes dynamic changes in Venus::HES5 microclusters

\section{3 between high and low states}

354 We hypothesised that the periodic microclusters of HES5 are generated through

355 Notch-Delta interactions that locally synchronise dynamic HES5 expression between

356 neighbouring cells. To test this, we treated spinal cord slice cultures with the Notch

357 inhibitor DBZ and performed kymograph analysis in the apical region of DMSO and

358 DBZ treated slices. In Notch inhibitor conditions, the HES5 levels reduce

359 continuously over time (Figure 4a) indicating that the DBZ is effective. The most

360 noticeable difference in the spatiotemporal HES pattern was that the temporal

361 transitions of microclusters from high to low Venus::HES5 were impaired by DBZ.

362 We saw fewer changes in the phase of the spatial periodic Venus::HES5 pattern

363 indicating the spatial pattern remained stable (Figure 4b,c and Figure EV3a,b). This

364 was quantified with a phase synchronisation index (see Methods), where low values

365 indicate the presence of phase changes at the same D-V locations (Figure $4 \mathrm{~d}$ ). The

366 phase synchronisation index was significantly higher in DBZ treated tissue indicating

367 that in the absence of Notch signalling, HES5 microclusters were more persistent in

368 the same region and that the dynamic changes in Venus::HES5 microclusters

369 between high and low levels are mediated by Notch signalling. The phase detection 
370 method (Hilbert transform) is not dependent on the level of expression and so the

371 reduction in HES5 levels in DBZ does not affect the analysis of microcluster high-to-

372 low and low-to-high phase switches.

373 We analysed the spatial periodicity of HES5 and found that the amplitude between

374 high and low microclusters appears diminished compared to control DMSO treated

375 conditions (Figure 4e). Spatial periodicity could be detected at the start of the movie,

376 immediately after DBZ addition, however the spatial periodicity was gradually

377 extinguished through loss of Venus::HES5 levels and spatial amplitude death

378 (Figure EV3c). Approximately 45\% of the DBZ treated slices did not show significant

379 peaks in the auto-correlation of detrended spatial Venus::HES5 profile by 10-12 hrs

380 of treatment (Figure $\mathbf{4 f}$ ) whereas periodicity was maintained in all DMSO conditions.

381 Spatial periodicity in detrended Venus::HES5 levels that could be detected in DBZ

382 treatment at early time points frequently appeared higher in Notch inhibitor treated

383 ex-vivo slices than in DMSO control (Figure $\mathbf{4 g}$ and Figure EV3d). Cell density also

384 decreased in Notch inhibitor conditions suggesting this increase in spatial period was

385 partially due to changes in the spatial arrangement of cells (Figure EV3e).

387 We also investigated how Notch inhibition may affect ultradian dynamics at single 388 cell level. We had previously reported that under DBZ conditions, single neural 389 progenitors continue to show oscillations and fluctuations in HES5 before undergoing 390 amplitude death (Manning et al., 2019b). However here we wanted to interrogate 391 how DBZ affects the way cells co-ordinate their activity in the tissue. To do this we 392 used the Kuramoto Order Parameter (KOP, also known as mean-field value) a 393 population measure of synchrony (Choi et al, 2000). High KOP levels close to 1 are 394 indicative of global in-phase activity whereas low KOP values close to 0 are 395 indicative of no in-phase synchrony (see Methods). We found that KOP of single 396 progenitors indicating weak levels of synchrony under DMSO conditions (Appendix

397 Figure S4e, mean 0.36) consistent with our findings of local in-phase activity but not 398 indicative of global synchrony. Furthermore, we observed a significant reduction of 399 KOP values in DBZ conditions (Appendix Figure S4e, mean 0.15).

401 Taken together, these findings show that Notch signalling is responsible for certain 402 aspects of the pattern, such as the dynamic switching between high/low HES5 403 microcluster states over time. However, inhibition of Notch does not seem to abolish 
404 the existence of microclusters or their spatial periodicity, as they can still be detected

405 until amplitude death occurs and the HES5 levels are depleted. At single cell level,

406 we observe that Notch signalling is likely to promote local in-phase ultradian co-

407 ordination between cells within a microcluster.

A model of Notch-Delta with HES5 auto-repression containing stochasticity and delays re-capitulates the existence of local in-phase HES5 dynamics

412 We used computational modelling to help us understand how positively correlated,

413 spatially periodic, and dynamic microclusters of cells may emerge in the spinal cord.

414 At single cell level, HES5 protein expression oscillations are due to HES5 self-

415 repression, an intra-cellular transcriptional time delay $\left(\tau_{H}\right)$ and short protein and 416 mRNA half-lives (Jensen et al, 2003; Momiji \& Monk, 2008; Monk, 2003). We

417 represented the auto-repressive interactions between HES5 mRNA and protein

418 using stochastic differential equations with time delay, as previously described in

419 (Galla, 2009; Manning et al., 2019a; Phillips et al., 2016). This single cell model has

420 been shown to faithfully recapitulate statistics of single cell HES5 expression

421 dynamics collected from spinal cord tissue (Manning et al., 2019a). We extended the

422 single-cell mathematical description of HES5 to a coupled dynamical model by

423 incorporating a repressive interaction in the form of a Hill function, that describes

424 how HES5 protein in one cell represses Hes5 transcription in a neighbouring cell via

425 Delta-Notch signalling (Figure 5a and Figure EV4a). We introduce the following set

426 of inter-cellular parameters (Figure 5b and Materials and Methods): (i) inter-cellular

427 time delay, representing the time required to transfer the signal from one cell to

428 another, that is, the time required for a change in HES5 protein in one cell to affect

429 Hes5 transcription in a neighbouring cell through Notch-Delta; (ii) the inter-cellular

430 repression threshold, representing the amount of HES5 protein required to reduce

431 Hes5 transcription in a neighbouring cell by half; the inter-cellular repression

432 threshold is inversely proportional to coupling strength where higher coupling

433 strength (or low inter-cellular repression threshold) indicates that less protein is

434 needed to repress the neighbour's Hes5 transcription by $50 \%$; and (iii) inter-cellular

435 Hill coefficient indicating how steep the response curve of Hes5 transcription is in

436 response to a change in HES5 protein in the neighbouring cell, with higher values

437 corresponding to increased nonlinearity. Interactions between cells are considered in 
438 a hexagonal grid whereby each cell can interact with its immediate 6 neighbours and

439 repression between cells is calculated through the inter-cellular Hill function by

440 averaging HES5 protein abundance over 6 neighbours (Figure 5b,c and Materials

441 and Methods). Thus, we generated a comprehensive, multiscale and stochastic

442 model with time delays, representative of the Delta-Notch-Hes interactions in the

443 multicellular tissue environment.

444 We parameterised this multiscale HES5 model with previously determined 445 experimental measures of HES5 protein and mRNA stability and with parameter 446 values of the single cell HES5 self-repression loop that can reproduce single neural 447 progenitor HES5 dynamics (see Methods and Appendix Table S3 Main), as 448 identified through Bayesian inference in our previous work (Manning et al., 2019a).

449 We then investigated the parameter space of unknown model parameters that are 450 characteristic of cell-to-cell interactions, namely the repression threshold (inverse of 451 coupling strength) and time delay, to identify values that are compatible with the 452 temporal period and phase synchronisation level of single cell Venus::HES5 453 expression dynamics (Figure 5d,e). The mean temporal period of Venus::HES5 in 454 ex-vivo spinal cord tissue is approx. 2-6 hrs (mean $3.3 \mathrm{hrs}$ ) (Manning et al., 2019a), 455 which could be reproduced by the model in a wide range of coupling strength and 456 inter-cellular time delay values (Figure 5d,f). We measured the temporal phase 457 synchronisation between single Venus::HES5 expressing cells in the apical region 458 and we found that the KOP was between 0.15 and 0.4 (Appendix Figure S4f, mean 459 0.3) consistent with KOP in the DMSO data (Appendix Figure S4e, mean 0.36). 460 This measure aided us in further reducing the parameter space of repression 461 threshold and inter-cellular time delay that could fit the observed data (Figure 5e,f). 462 The accepted parameter values for inter-cellular time delay were consistent with a 463 Delta to HES5 transmission time of 128 min measured experimentally (Isomura et al, 464 2017). A Hill coefficient value larger than 2 was required for notable synchrony to 465 emerge $(\mathrm{KOP}>0)$ and only minor differences in terms of parameter selection were 466 observed for values between 3 and 6 (Appendix Figure S5a).

467 This parameter exploration allowed us to optimise the search for spatial patterns that 468 emerge at different coupling strengths using kymograph analysis (Figure $\mathbf{5 g}, \mathbf{h}$ ). We 469 set the inter-cellular time delay to $150 \mathrm{~min}$ and Hill Coefficient to 4 (Methods) and 470 then compared the synthetic HES5 spatiotemporal characteristics at specific 471 coupling strength levels (parameter space indicated by the white box in Figure 5e). 
472 Our comparison showed that strong coupling (i.e. high coupling strength or low inter-

473 cellular repression threshold) induces Alternating high-low dynamics whereby single

474 neighbouring cells adopt either high oscillatory HES5 or stable low HES5 in an

475 alternating spatial pattern that does not evolve over time (Figure 5g, Alternating

476 high-low, Appendix Movie $\mathbf{S 2}$ first panel). Meanwhile at mid-level coupling, the

477 multiscale model induces globally synchronised oscillations in all cells (Figure $\mathbf{5 g}$,

478 Global in phase and Appendix Movie S2 second panel). At weak coupling

479 strength, the spatial patterns show areas of local synchronisation emerging between

480 neighbouring cells (Figure 5g, Local in phase and Appendix Movie S2 third panel)

481 resembling activity observed in tracked single cell pairs in experimental data (Figure

482 3k). Under no coupling conditions we observed autonomous non-synchronised

483 stochastic oscillations and fluctuations across the tissue (Figure $5 \mathbf{h}$ and Appendix

484 Movie S2 fourth panel). These observed changes in synchronisation are indicated

485 by population KOP values (Figure $\mathbf{5 g}$ ) and we further confirmed that the KOPs

486 correspond to changes in synchrony in terms of single cell expression dynamics

487 between neighbouring cells (Figure 5i). As expected, in the uncoupled cells we

488 observed no synchrony $(\mathrm{KOP} \approx 0)$ and activity in neighbouring cells was un-

489 coordinated over time (Figure $\mathbf{5 h}, \mathbf{j}$ ). Therefore the model can recapitulate the local

490 in-phase behaviour in Venus::HES5 observed between single cell pairs in a

491 microcluster.

492 Our explorations of synthetic data show that at weak coupling strength microclusters

493 consisting of in-phase cells can be generated in the model with a diameter of 2-6

494 cells (Appendix Figure S5b and Methods), consistent with cluster size in spinal

495 cord tissue. However, the occurrence rate of microclusters was low, as these were

496 observed around $20-30 \%$ of the time, although still higher than in the uncoupled

497 situation (Appendix Figure S5c and Appendix Figure S6a). Thus, weak coupling

498 conditions generate microclusters by promoting in-phase activity between

499 neighbouring cells, however these appear transiently and with low probability. In

500 addition, the microclusters of locally synchronised cells were not spatially periodic

501 (Appendix Figure 5d and Appendix Figure 6b). As expected, at high coupling (low

502 repression threshold) we detected an alternating pattern of HES5 with a spatial

503 periodicity of 2 cells, which is a characteristic of the classic lateral inhibition

504 alternating high-low pattern (Appendix Figure S5d and Appendix Figure S6b). 
505 In conclusion, our multicellular coupled model shows that spinal cord progenitors can

506 locally synchronise at weak coupling strength to generate microclusters of 2 to 6

507 cells in diameter, a similar size to those seen in tissue, (Figure 1d) with single cell

508 Venus::HES5 expression dynamics consistent with previous reports (Manning et al.,

509 2019a). However, the model cannot recapitulate the repeated spatial co-ordination

510 and continuous presence of dynamic microclusters, suggesting that additional

511 mechanisms may act in the tissue environment to stabilise their presence and

512 promote spatially periodic emergence.

513 The model predicts that probability of differentiation is regulated by the 514 coupling strength between cells

515 To understand how the spatial pattern of HES5 and dynamic micropatterns in

516 particular may affect properties of neurogenesis, we made the assumption that when

517 HES5 is low, there is increased probability that the cell would differentiate consistent

518 with findings that differentiation is accompanied by switching off of HES5, a

519 repressor of neurogenesis (Bansod et al., 2017; Manning et al., 2019a; Sagner et al.,

520 2018). We introduced a "differentiation threshold", which was set at the level of the

521 HES5 population mean for each simulation ( Figure 5i, Population Mean) and we

522 reasoned that if expression level in a cell dropped below this threshold there was an

523 increasing probability to switch off HES5 and differentiate (Figure 6a). We found that

524 at high coupling strength (Alternating high-low conditions) the probability to

525 differentiate is the highest, whereas medium and weak coupling strength

526 (corresponding to Global and Local in phase synchronisation, respectively) had

527 progressively lower probability of differentiation (Figure 6b).

528 To understand why this is happening, we looked at the Coefficient of Variation (CoV,

529 Figure 6c), a measure of variability denoting standard deviation over the mean. We

530 investigated both the temporal (Temporal CoV) and spatial variation (Spatial CoV) in

531 simulated HES5 expression. Indeed, both temporal (indicative of single cell

532 amplitude) and spatial CoV (indicative of variation between HES5 high and low

533 regions in space) appear highest in Alternating high-low conditions and lowest

534 for Local in phase micropatterns (Figure 6c). However, we found that changes in

535 spatial CoV correlated better with changes in rate of differentiation, especially at low

536 repression threshold/high coupling strength (Figure $6 \mathbf{c}$ versus Figure $6 \mathbf{b}$ Alternating

537 high-low). Thus, our model predicts that the strength of cell:cell coupling may 
538 increase the probability of differentiation through amplifying cell:cell differences in

539 abundance which in turn affects how far the cells dip below the threshold of

540 differentiation.

541

542 In tissue, HES5 spatial pattern varies predictably with the rate of differentiation

543 To test the computational prediction that the spatial pattern of HES5 (determined by

544 the coupling strength) regulates the probability of differentiation, we compared the

545 pattern in motorneuron and interneuron progenitor domains. We chose this

546 comparison because at E10.5 the motorneuron domain is known to have a higher

547 differentiation rate than the interneuron domain (Kicheva et al, 2014), therefore one

548 would expect a different HES5 spatial pattern. We stained for the motorneuron

549 progenitor marker OLIG2 (Figure 7a and Figure EV4b,c) and analysed expression

550 levels of Venus::HES5 and Neurogenin 2 (NGN2) in the two domains. The

551 motorneuron domain had lower HES5 levels and higher NGN2 levels than the

552 interneuron domain (Figure 7b) consistent with the opposing activity of these genes

553 on cell differentiation (Imayoshi \& Kageyama, 2014).

554 We then used nuclear segmentation and pseudo-color analysis of mean

555 Venus::HES5 intensity per nucleus (Figure 7c) and found that the interneuron

556 domain shows the presence of microclusters mainly consisting of 2-3 cells wide in

557 the dorsal to ventral axis whereas in the motoneuron domain high Venus::HES5 cells

558 were mainly found as single cells, alternating with cells expressing lower

559 Venus::HES5 (Figure 7d). We validated this finding further by investigating spatial

560 periodicity by domain in live tissue slices. The domain border between motorneuron

561 and interneuron progenitors was $35 \mu \mathrm{m}$ ventral to the peak of HES5 expression

562 (Figure EV4d) allowing us to correctly identify the two domains without the need for

563 an OLIG2 reporter in the same tissue. We found that spatial periodicity was reduced

564 in the motorneuron compared to the interneuron domain when analysed using both

565 peak to peak distance in auto-correlation (Figure 7e, MN mean $31 \mu \mathrm{m}$ vs IN mean 41

$566 \mu \mathrm{m}$ and Figure EV4e,f) and dominant spatial period by Lomb-Scargle periodogram

567 (Figure EV4g, MN mean $25 \mu \mathrm{m}$ vs IN mean $40 \mu \mathrm{m}$ ). Thus, both nuclear

568 segmentation analysis and spatial periodicity indicated that, in the interneuron

569 domain, microclusters of 2-3 cells are found in a spatially periodic pattern repeated

570 every 4 cells. Meanwhile, the motorneuron domain shows alternating high and low 
571 HES5 levels between neighbouring cells and a significant reduction in spatial

572 periodicity, both of which are pointing to the motorneuron domain more closely

573 resembling Alternating high-low conditions.

574 The model predicts that the coupling strength regulates the type of spatial micro-

575 patterning hence, we hypothesised that the interneuron and motorneuron domains

576 have different coupling strength. The model indicates that weak coupling, likely to be

577 characteristic of the interneuron domain, would generate smaller cell-cell

578 concentration differences compared to strong coupling (Appendix Figure S7a). This

579 is because weakly coupled cells have less ability to repress the transcription of their

580 neighbours and so are more similar in levels. This relationship should persist even

581 after correcting for mean level in each condition. We have previously used

582 fluorescence correlation spectroscopy (FCS) to generate a spatial map of nuclear

583 Venus::HES5 concentration in the E10.5 spinal cord (Manning et al., 2019b). Using

584 this data, we calculated the difference in Venus::HES5 concentration between

585 neighbouring cell-pairs relative to the mean by domain and indeed found that it is

586 lower in the interneuron domain compared to the motorneuron domain (Figure 7f).

587 The correction by mean was important as variability in expression is expected to

588 scale with the mean. This finding was confirmed by measuring the spatial amplitude

589 of Venus::HES5, which was also higher in the motorneuron domain (Figure EV4h).

590 These findings are consistent with the notion that the coupling strength in the IN

591 domain is lower than in MN one. Taken together, these results show that interneuron

592 progenitors are more likely to be found in a locally synchronised state through weak

593 coupling which correlates with a lower rate of differentiation. By comparison,

594 progenitors in the motorneuron domain are mostly found in alternating high-low

595 pattern and show a higher rate of differentiation, as predicted computationally by a

596 higher coupling strength.

\section{NGN2 expression is spatially periodic and coordinates with the HES5 pattern}

599 Given that the spatial pattern of HES5 is relevant to the rate of neurogenesis we 600 investigated the wider applicability of our findings by characterising the spatial 601 patterns of other genes in the Notch-Delta gene network. Chromogenic in-situ 602 hybridisation of Dll1 and Jag1 mRNA shows that DIl1 has a broad expression 603 domain that covers the motor neuron domain and the ventral-most part of the 604 interneuron domain (Figure EV5a) (Marklund et al, 2010). Alternate stripes of Jag1 
605 and Dll1 are observed in the intermediate spinal cord, which covers the remaining 606 part of the interneuron domain (Figure EV5a) (Marklund et al., 2010). We have 607 performed smiFISH for DI/1 to get a high-resolution understanding of DIl1 expression 608 pattern in the interneuron domain where HES5 is expressed in microclusters. We 609 found that DIl1 expression is non-uniform and appeared in microstripes of a few cells 610 (Figure EV5b,c), suggesting that other genes show similarities in local spatial 611 patterning.

612 We next analysed the spatial expression pattern of the pro-neural factor NGN2. 613 Using both NGN2 antibody staining and a NGN2:mScarlet fusion reporter mouse, we 614 found that NGN2 also has a spatially periodic expression pattern, with around half 615 the spatial period of Venus::HES5 (Figure 8a-c). The spatial period of NGN2 is 616 smaller in the motorneuron domain with a mean period of $21 \mu \mathrm{m}$ supporting the 617 conclusion that NGN2 spatial expression patterns are different between motorneuron 618 and interneuron domains (Figure 8d). To understand how the NGN2 and 619 Venus::HES5 periodic patterns map on to each other we used the cross-correlation 620 function of the NGN2 and Venus::HES5 spatial profile from the same tissue (Figure $621 \mathbf{8 e , f ) . ~ T h e ~ c r o s s - c o r r e l a t i o n ~ a n a l y s i s ~ s h o w e d ~ t h e ~ p r e s e n c e ~ m u l t i p l e ~ p e a k s ~ i n d i c a t i n g ~}$ 622 coordination between the two signals that was not reflected in the brightfield control 623 (Figure 8f). As expected for signals of different periodicities, we observed primary 624 peaks indicative of positively correlated activity (Figure 8f, red arrowheads) as well 625 as secondary peaks indicative of negatively correlated activity (Figure $8 \mathbf{g}$, black 626 arrowheads). The cross-correlation is also indicative of whether peaks of activity are 627 present in the same area. To ascertain this we performed a phase-shift analysis by 628 measuring phase shift as the absolute lag corresponding to the primary cross629 correlation peak closest to lag 0 . In Figure $8 \mathrm{f}$ a dominant peak falls close to lag 0 630 thus indicating that NGN2 and Venus::HES5 patterns coordinate in the same region. 631 Thus we concluded that NGN2 shows a spatial periodic pattern of half the period of 632 Venus::HES5 resulting in half of the NGN2 high cells occurring in HES5 high 633 microclusters and half in HES5 low.

635 Furthermore when we performed phase-shift analysis in multiple cross-correlation 636 examples (Methods), the shift was minimal and consistently less than a single-cell 637 width (Figure $\mathbf{8 g}$ ). This strongly pointed to coordination not only in the same region 
638 but also in the same cells. We subsequently investigated this by using single nuclear 639 segmentation of high resolution images to visualise the NGN2-HES5 spatial 640 relationship. Indeed we found that within a HES5 microcluster in the interneuron 641 domain, only 1-2 cells (and in the MN domain only one cell per cluster) show high 642 NGN2 expression levels (Figure 8h). As high NGN2 is an early marker of 643 differentiation, this suggests that similar to the mathematical model (Appendix

644 Figure S7b) cells in a cluster do not differentiate in unison; instead microclusters 645 may act to select a cell for differentiation, hence regulating spatial and temporal 646 aspects of neurogenesis.

\section{Discussion}

649 In this paper, we have addressed how cells co-ordinate their decisions with that of 650 their neighbours so that neurogenesis takes place at a pace appropriate for the 651 anatomical location. We have investigated the fine-grained pattern of neurogenesis 652 in the spinal cord by monitoring the spatiotemporal patterning of key progenitor TF 653 HES5 using live imaging analysis that is optimised towards revealing coordinated 654 tissue level behaviour that would not otherwise be evident. In combination with 655 computational modelling it enabled a multi-scale synthesis of the data with predictive 656 power. We have uncovered an unexpected 3-tiered spatial and temporal 657 organisation, which we discuss below in an ascending order of complexity.

658 First, within the ventral HES5 expression domain, which encompasses distinct MN 659 and IN domains, we have discovered clusters of cells with positively corelated HES5 660 expression levels. These clusters, described for the first time here, are 2-3 cells wide 661 in D-V and 3-4 cells wide in A-B axes, hence termed microclusters. To detect 662 microclusters, we removed longer range spatial trends such as overall gradients of 663 intensity in HES5 expression (which have not been dealt further here) allowing us to 664 concentrate on local correlations of expression. By following Venus::HES5 in pairs of 665 single cells in proximity we find that microclusters are a composite of positive 666 correlations in levels of Venus::HES5 and locally synchronised (in-phase) HES5 667 dynamics. We also found that the microcluster organisation extends to DLL1 668 although we have not been able to study it with live imaging in this work. The 669 clustering organisation was surprising because previous studies have suggested that 670 in neurogenesis oscillators are in anti-phase in neighbouring cells (Kageyama et al, 671 2008; Shimojo et al., 2016; Shimojo \& Kageyama, 2016). DLL1 oscillations were 
672 observed with live imaging in tissue but only 2 neighbouring cells were monitored in 673 close vicinity and a single example shown for anti-phase oscillations, which may

674 explain the contradictions with our data. Thus, the discrepancy could be to a 675 difference in scale of analysis or perhaps to the different molecules studied.

676 Second, we have found that HES5 microclusters are arrayed in a spatially periodic 677 pattern along the D-V axis of the ventral HES5 domain, meaning that high and low 678 HES5 expression clusters alternate regularly in space. We also found that NGN2 is 679 expressed periodically along the D-V axis with half the periodicity of HES5 such that 680 NGN2 high cells are found both within HES5 high and low microclusters. SmiFISH 681 showed DIl1 expressed in microstripes but the images of single Dll1 mRNA 682 molecules were not amenable to auto-correlation thus, it is not known whether they 683 occur on the same spatial scale as HES5. Multiple stripes of DIl1 and Jag1 and Lfng 684 have been observed, but at the larger progenitor domain scale (Marklund et al., 685 2010; Ramos et al, 2010). Such spatial periodicity at a fine level within the ventral 686 HES5 domain contrasts with the large-scale organisation of HES5 in 2 separate 687 broad domains along the D-V axis (Sagner et al., 2018).

688 Thirdly, the HES5 spatial pattern of microclusters was not static but appeared 689 dynamic over time; High HES5 expressing microclusters persisted for 6-8 hrs and 690 then switched to low expression, while low expressing microclusters showed the 691 opposite behaviour. In other words, high and low expressing microclusters alternated 692 and sometimes created phase waves that travelled through the tissue over time.

693 These waves are somewhat reminiscent of phase waves of LFNG and AXIN2 694 expression that are observed in somitogenesis (Baron \& Galla, 2019; Sonnen et al, 695 2018; Tsiairis \& Aulehla, 2016) however, in the spinal cord such phase waves were 696 weak. This complex spatial and temporal dynamic pattern of HES5 in spinal cord 697 generated two important questions: how might it be generated and what might it 698 mean for neurogenesis? Knowing that Hes genes and HES5 in particular, are 699 activated by Notch signalling, we treated ex-vivo spinal cord tissue with DBZ to 700 disrupt Notch signalling. We observed that the Notch inhibitor treatment extinguished 701 spatial periodicity gradually and slowly, over a period of $12 \mathrm{hrs}$, concurrent with HES5 702 level downregulation. This is consistent with the amplitude death that we observed in 703 single cell data under the same treatment (Manning et al., 2019a). The effect of 704 Notch inhibition was far more pronounced in the temporal nature of the pattern; in 705 the absence of Notch signalling, the HES5 spatially periodic pattern of low and high 
706 expressing microclusters became "frozen" in time. These findings suggest that Notch

707 signalling plays a part in making the pattern dynamic over time but cannot account

708 for the entire spatiotemporal complexity of HES5 expression that we see ex vivo.

709 Computational modelling helped us to explore further the role of Notch in generating

710 the spatiotemporal pattern of HES5 expression. We have used a simplified

711 multiscale stochastic model of HES5 self-repression and inter-cellular coupling with

712 delay, parameterised on our own experimental data, namely the single cell HES5

713 temporal period and extent of HES5 expression synchronicity between cells using

714 the KOP. With this model we were able to explore the influence of the coupling

715 strength between cells in producing spatiotemporal HES5 expression patterns. We

716 found multiple spatiotemporal patterns, namely; an alternating high and low pattern

717 (at high coupling strength), global tissue synchronisation (at mid coupling strength),

718 and un-coordinated pattern (at no coupling), see Appendix. Movie S2. Importantly,

719 at weak coupling strength and intercellular time delay that is consistent with previous

720 reports, we observed the emergence of dynamic microclusters that matched our

721 experimental observations. The emergence of dynamic patterns that do not resolve

722 into steady HES "on" or "off" static patterns has been previously observed in a

723 stochastic multicellular tissue model combining Notch-Delta and Hes auto-repression

724 but not confirmed in tissue (Tiedemann et al, 2017). However, the dynamic

725 microclusters in our model occurred infrequently (with a probability of 20-30\%) even

726 though the model takes into consideration stochasticity and time delays; two features

727 that represent the tissue context well. The low frequency of clusters did not improve

728 after detailed optimised exploration of parameter space, which led us to conclude

729 that a Notch based cell-to-cell signalling with the assumptions we have made,

730 recapitulates only part of the observed pattern in vivo. Extension of the model to

731 include i) longer range cell-cell interaction via cytonemes, or due to the elongated

732 shape of the progenitor cells, ii) increased complexity of the gene network such as

733 cis-inhibition between Delta-Notch or differences in signalling between different

734 Notch ligands, may be able to increase the fidelity of microcluster emergence.

735 Indeed, it has been shown that such modifications increase the range of spatial

736 patterns that can be obtained (Boareto et al, 2015; Cohen et al., 2010; De

737 Joussineau et al, 2003; Hadjivasiliou et al, 2019; Petrovic et al, 2014; Sprinzak et al,

738 2010). Other ways in which the model can be extended is to incorporate the 
739 influence of morphogen signalling gradients along the $\mathrm{D}-\mathrm{V}$ axis or differentiation

740 gradients along the A-B axis, as these are known to exist in the tissue.

741 Nevertheless, the computational model we developed, allowed us to explore the 742 advantages that organisation in dynamic microclusters may offer as a developmental 743 strategy for neurogenesis in the embryonic spinal cord. Overall, we found that the 744 spatiotemporal HES5 pattern was affected by the coupling strength between cells 745 and in turn, affected the rate of differentiation. Based on our findings we propose that 746 a classic lateral inhibition alternating high-low HES5 pattern (achieved at high 747 coupling strength) shows the highest rate of differentiation because it generates two 748 HES5 states ("on" and "off") in a spatially-alternating pattern and this is likely to result 749 in tipping of more cells towards differentiation. Global synchronisation (medium 750 coupling strength) shows a medium rate of differentiation; however this regime is not 751 observed in spinal cord data perhaps because the synchronous differentiation in 752 "blocks" of cells found close by in tissue, although an appropriate developmental 753 strategy for somitogenesis, may be incompatible with the structural integrity of the 754 neural tissue or the finer diversification of neuronal fates within each domain. The 755 un-coordinated pattern (no coupling between cells) has similar rates of differentiation 756 as weak coupling, however, weak coupling strength is advantageous because it 757 allows local in phase synchronisation, which by analogy to global synchronisation 758 (Figure 6c, Global vs Local in phase), appears to transiently increase the amplitude 759 of temporal oscillations in HES5 expression (Figure 5i, panel 3-Local in phase). This 760 is important because a transient amplitude increase (due to the presence of 761 microclusters at Local in phase conditions) could faciliate the progression to 762 differentiation. Indeed, we have previously shown that HES5 oscillations in 763 proliferating spinal cord progenitors have low amplitude and show mainly aperiodic 764 fluctuations (noisy dynamics) but the propensity to oscillate as well as the peak-to765 trough amplitude increases as cells enter the differentiation pathway (Manning et al., 766 2019a). We have also shown that when the transition from noisy dynamic 767 expression to oscillatory expression does not take place, progenitor cells are unable 768 to downregulate HES levels and differentiate (Soto et al., 2020). We speculate that 769 microclusters may act to reliably select one or two cells that go on to express NGN2 770 and differentiate and that the spatial periodicity of microclusters may space out 771 differentiating cells to maintain tissue organisation. 
772 We tested the model hypothesis that by changing the HES5 spatiotemporal pattern

773 through tuning the coupling strength, the tissue is able to fine tune the rate of

774 neurogenesis. We compared the motorneuron and interneuron progenitor domains

775 as these two neighbouring domains in the D-V axis are known to have different rates

776 of differentiation (Kicheva et al., 2014; Kuzmicz-Kowalska \& Kicheva, 2020). Indeed,

777 we find that that in the $\mathrm{MN}$ domain where the rate of differentiation is highest at

778 E10.5, the HES5 and NGN2 pattern most closely matches the alternating high-low

779 pattern (Figure 8i, MN). In the ventral interneuron domain, we propose that the local

780 in phase synchronisation pattern (predicted to occur at weak coupling strength) is the

781 closest match to the ex-vivo situation (Figure 8i, IN). We propose it represents a

782 strategy to balance prolonged neurogenesis, with a reasonable rate of differentiation

783 and a transient increase in oscillation amplitude that is suitable for decoding by

784 downstream genes. There may be additional molecular differences between the

785 motorneuron and interneuron domains that regulate the rate of differentiation.

786 Indeed, the transcription factor OLIG2 is expressed in the motorneuron domain and

787 has been shown to promote differentiation by directly inhibiting HES5 (Sagner et al.,

788 2018). We speculate that this mechanism could interplay or directly affect the cell-

789 cell coupling strength by changing HES5 levels or binding partners.

790 In conclusion, our findings show HES5 spatially periodic micro-patterns exist in the

791 developing spinal cord, they underlie the rate of neurogenesis and are an emergent

792 property of the multi-scale synthesis of dynamical gene expression and Notch

793 coupling. This temporally dynamic expression is a testament to the power of live

794 tissue imaging in providing mechanistic insights of complex phenomena as they

795 unfold in real time.

798 Materials and Methods

800 Animals

801 Animal experiments were performed by personal license holders under UK Home

802 Office project license PPL70/8858 and within the conditions of the Animal (Scientific

803 Procedures) Act 1986. Venus::HES5 knock-in mice (ICR.Cg-

804 Hes5<tm1(venus)Imayo>) were obtained from Riken Biological Resource Centre,

805 Japan and maintained as a homozygous line. In these mice the mVenus fluorescent 
806 protein is fused to the $\mathrm{N}$-terminus of endogenous HES5. Sox1Cre:ERT2 mice

807 (Sox1tm3(cre/ERT2)Vep were obtained from James Briscoe with the permission of

808 Robin Lovell-Badge. R26R-H2B::mCherry mice were obtained as frozen embryos

809 from Riken Centre for Life Science Technologies, Japan and C57BI6 mice were used

810 as surrogates. NGN2::mScarlet-I mouse was generated by the University of

811 Manchester Genome Editing Unit (see Appendix methods). The mScarlet-I

812 fluorescent protein is fused to the C-terminus of endogenous NGN2.

\section{Embryo slicing and live imaging}

815 E0.5 was considered as midday on the day a plug was detected. For matings with 816 R26R-H2B::mCherry Sox1Cre:ERT2, intra-peritoneal injection of pregnant females 817 with $2.5 \mathrm{mg}$ Tamoxifen (Sigma) was performed 18 hours prior to embryo dissection.

818 This enables single cell tracking through mosaic labelling of nuclei with 819 H2B::mCherry. Whole embryos were screened for H2B::mCherry expression using 820 Fluar 10x/0.5 objective on a Zeiss LSM880 confocal microscope. After decapitation, 821 embryo bodies were embedded in $4 \%$ low-gelling temperature agarose (Sigma) 822 containing $5 \mathrm{mg} / \mathrm{ml}$ glucose (Sigma). $200 \mu \mathrm{m}$ transverse slices of the trunk containing

823 the spinal cord around the forelimb region were obtained with the Leica VT1000S 824 vibratome and released from the agarose. Embryo and slice manipulation were 825 performed in phenol-red free L-15 media (ThermoFisher Scientific) on ice and the 826 vibratome slicing was performed in chilled 1xPBS (ThermoFisher Scientific).

827 For snapshot imaging of live E10.5 spinal cord, slices were stained with 50 $\mu$ M Draq5 828 (Abcam - ab108410) in 1xPBS (ThermoFisher Scientific) for 1.5hrs on ice if 829 required, and then placed directly on to a $35 \mathrm{~mm}$ glass-bottomed dish (Greiner 830 BioOne). Images were acquired with a Zeiss LSM880 microscope and C831 Apochromat 40x 1.2 NA water objective. E10.5 spinal cord slices for live timelapse 832 microscopy were placed on a $12 \mathrm{~mm}$ Millicell cell culture insert (MerckMillipore) in a $83335 \mathrm{~mm}$ glass-bottomed dish (Greiner BioOne) incubated at $37^{\circ} \mathrm{C}$ and $5 \% \mathrm{CO}_{2}$. The 834 legs of the cell culture insert were sanded down to decrease the distance from the 835 glass to the tissue. 1.5mls of DMEM F-12 (ThermoFisher Scientific) media containing $8364.5 \mathrm{mg} / \mathrm{ml}$ glucose, 1x MEM non-essential amino acids (ThermoFisher Scientific),

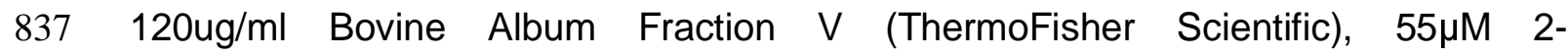
838 mercaptoethanol, 1x GlutaMAX (ThermoFisher Scientific), 0.5x B27 and 0.5x N2 was 
839 added. Movies were acquired using Zeiss LSM880 microscope and GaAsP

840 detectors. A Plan-Apochromat 20x 0.8 NA objective with a pinhole of 5AU was used.

$84110 \mathrm{z}$-sections with $7.5 \mu \mathrm{m}$ interval were acquired every $15 \mathrm{mins}$ for $18-24 \mathrm{hrs}$. DMSO

842 (Sigma) or $2 \mu \mathrm{M} \mathrm{DBZ}$ (Tocris) was added to media immediately before imaging.

\section{Single cell tracking over time}

844 Single neural progenitor cells in E10.5 spinal cord slices were tracked in Imaris on

845 the H2BmCherry channel using the 'Spots' function with background subtraction and

846 the Brownian motion algorithm. All tracks were manually curated to ensure accurate

847 single-cell tracking. Background fluorescence was measured via an ROI drawn on a

848 non-Venus::HES5 expressing region on the tissue and subtracted from spot

849 intensity. To account for any photobleaching and allow comparison of intensities

850 between movies the mean intensity of mCherry and Venus in each spot was

851 normalised to the mean intensity of mCherry or Venus in the whole tissue. The whole

852 tissue volume was tracked using the 'Surfaces' and 'Track over time' function. For

853 the calculation of Kuramoto order parameter from timeseries see Temporal phase

854 synchronisation.

856 Immunofluorescent staining

857 Trunks of E10.5 embryos for cryo-sectioning were fixed in $4 \%$ PFA for 1 hour at $4^{\circ} \mathrm{C}$,

858 followed by 3 quick washes with 1xPBS and 1 longer wash for 1 hour at $4^{\circ} \mathrm{C}$.

859 Embryos were equilibrated overnight in $30 \%$ sucrose (Sigma) at $4^{\circ} \mathrm{C}$ before

860 mounting in Tissue-Tek OCT (Sakura) in cryomoulds and freezing at $-80^{\circ} \mathrm{C} .12 \mu \mathrm{m}$

861 sections were cut on Leica CM3050S cryostat. E10.5 spinal cord slices cultured on

862 Millicell inserts were fixed in 4\% PFA for 4 hours. For staining, tissue and sections

863 were washed in PBS followed by permeabilisation in PBS 0.2\% Triton X-100 (Sigma)

864 and blocking with PBS 0.05\% Tween20 (Sigma) + 5\% BSA (Sigma). Primary and

865 secondary antibodies were diluted in PBS 0.05\% Tween20 + 5\% BSA. Tissue was

866 incubated with primary antibodies overnight at $4^{\circ} \mathrm{C}$, then washed three times for $5-$

86710 minutes in PBS 0.05\% Tween20, incubated with secondary antibodies and DAPI

868 (Sigma) for 6 hours at room temperature, and washed again three times in PBS-T.

869 Sections were mounted using mowiol 4-88 (Sigma). Primary antibodies used were

870 rabbit anti-SOX2 (ab97959, 1:200), rabbit anti-OLIG2 (EMD Millipore AB9610, 1:200)

871 and goat anti-NGN2 (Santa Cruz Biotechnology sc-19233, 1:200). 


\section{3 smiFISH probe design and synthesis}

874 The smFISH probes were designed using the probe design tool at 875 http://www.biosearchtech.com/stellarisdesigner/. Depending on the GC content of 876 the input sequence, the software can return varied size of probes, 18 and 22nt, 877 hence giving the largest number of probes at the maximum masking level. It also 878 uses genome information for the given organism to avoid probeswith potential 879 off-target binding sites. Using the respective gene mature mRNA sequence, we 880 designed 36 probes for Hes5 and 48 probes for DIl1 (Tables $\mathrm{X}$ and $\mathrm{Y}$ ) and added a 881 FLAP sequence (5'-CCTCCTAAGTTTCGAGCTGGACTCAGTG-3') to the 5' of each

882 gene-specific sequence (IDT). The designed set of probes were labelled with Quasar 883670 (Biosearch Technologies) for Hes5 and CalFluor 610 (Biosearch Technologies) 884 for Dll1 following the protocol from Marra et al. 2019

\section{smiFISH on mouse sections}

887 smiFISH protocol for mouse section embryos was developed by adapting smiFISH

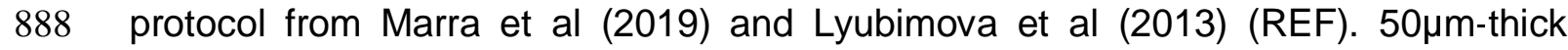
889 sections of E10.5 spinal cord were collected and transferred onto superfrost glass 890 slides (VWR $631-0448$ ) and kept at $-80^{\circ} \mathrm{C}$. Sections were left at room temperature to 891 dry for 5-10 min and then fixed in 4\% formaldehyde in 1× PBS followed by two quick 892 washes in 1XPBS. 1:2000 dilution of proteinase $\mathrm{K}$ (20mg mL-1stock) in 1X PBS was 893 pipetted onto each slide and left for 5-10 min followed by two washes in 2X SCC.

894 Sections were then incubated at $37^{\circ} \mathrm{C}$ twice in wash buffer $(5 \mathrm{ml}$ of $20 \times \mathrm{SSC}, 5 \mathrm{ml}$ of 895 formamide and $45 \mathrm{ml}$ of deionized, nuclease-free water). 250uL of hybridization 896 buffer (1 $\mathrm{g}$ dextran sulfate, $1 \mathrm{~mL}$ 20X SSC, $1 \mathrm{~mL}$ deionized formamide, $7.5 \mathrm{~mL}$ 897 nuclease-free water) with 100-240nM the fluorescent smiFISH probes was pipetted 898 onto each slide and incubated overnight at $37^{\circ} \mathrm{C}$ in a humid container shielded from 899 light. Samples were then washed as follows: twice in wash buffer at $37^{\circ} \mathrm{C}$ for $3 \mathrm{~min}$, 900 twice in wash buffer at $37^{\circ} \mathrm{C}$ for $30 \mathrm{~min}$, one wash in $1 \mathrm{X}$ PBS at room temperature for

$9015 \mathrm{~min}$. After smiFISH staining sections were washed for $2 \mathrm{~min}$ in PBS and mounted 902 using Prolong Diamond Antifade Mountant with DAPI (Thermo Fisher P36962). 
905 smiFISH images were collected with Leica TCS SP8-inverted confocal microscope

906 using objective HC PL APO CS2 40x/1.30oil. We acquired three-dimensional stacks

$9072048 \times 1024$ pixels and $z$ size $0.4 \mu \mathrm{m}$. The voxel size was $0.19 \times 0.19 \times 0.4 \mu \mathrm{m}$. Quasar

908670 and CalFluor 610 were imaged with pinhole 1 Airy Unit. Channels were

909 sequentially imaged. Deconvolution of confocal images was performed using

910 Huygens Professional Software. As pre-processing steps, the images were adjusted

911 for the "microscopic parameters" and for additional restoration such as "object

912 stabilizer"; the latter was used to adjust for any drift during imaging. Following, we

913 used the deconvolution Wizard tool, the two main factors to adjust during

914 deconvolution were the background values and the signal-to-noise ratio. Background

915 was manually measured for every image and channel, while the optimal

916 signal-to-noise ratio identified for the images was value 3. After deconvolution, the

917 images were generated with Imaris 9.3

918

919 Microcluster quantification and correlation of nuclear Venus::HES5 intensity

920 with distance and neighbours

921 Individual Draq5+ nuclei were manually segmented as ellipses using ImageJ,

922 converted to a mask and subsequently eroded using the ImageJ function 'erode' to

923 ensure no overlap between nuclei. Small nuclei with very high Draq5 intensity were

924 removed to avoid dead or dying cells. The mean intensity of each nuclear ROI was

925 plotted using the 'viridis' (Venus::HES5) or 'magma' (NGN2) LUT. Microcluster size

926 was calculated by quantile normalising the distribution of Venus::HES5 intensities

927 across experiments, then counting the maximum number of adjacent cells in the

928 highest colour band of the viridis LUT. The centroids of the manually segmented

929 cells were used to measure distance and hence rank between neighbours and a

930 correlation of the distance and mean nuclear Venus::HES5 intensity was calculated

931 using the 'corr' function in MATLAB. Mean nuclear Venus::HES5 intensity was also

932 randomised between nuclei and repeated 100 times before undergoing the same

933 distance vs mean intensity correlation.

934

935 Centre of intensity detection and radial gradient analysis

936 The centre of intensity (COI) was calculated using a centre of mass approach. The

937 intensity of each nuclei was multiplied by their position. These were then summed 
938 and divided by the sum of all nuclear intensities. The COI was used to sort cells in to

9395 equally spaced radial zones with increasing distance from the COI. The mean

940 Venus::HES5 intensity of nuclei in these zones was calculated and then subtracted

941 from each nucleus in that zone to remove the radial gradient. A simulated radial

942 gradient from a single focal point in the image was generated using

$943 \quad I_{r}=Z+\alpha X_{r}$

945 Where $\mathbf{I}_{\mathbf{r}}$ is the new intensity of the cells, $\mathbf{Z}$ is simulated intensities with the mean and

946 variance similar to that of real data, $\alpha$ is the gradient strength parameter and $x_{r}$ is a

947 function of the distance from the centre of intensity. As $\alpha$ increases the radial

948 gradient is less affected by random deviation in HES5 expression.

950 Detection and periodicity analysis of spatial expression patterns

951 To remove the possibility that changes in cell positions lead to shifts in the 952 kymograph stripes and artifacts in the dynamic analysis, movies underwent image

953 registration to account for global tissue drift and were subject to strict quality controls

954 for local tissue deformation. The average motility of tracked single cells over time in

955 the D-V axis was compared to patterns/waves of Venus::HES5 intensity in the 956 kymograph and included a maximum threshold of the averaged single cell 957 displacement (see Appendix Table S1). Bleach correction was then performed 958 using a ratiometric method in ImageJ.

959 Spatial expression profiles and kymographs were generated in Zen Blue (Carl Zeiss 960 Microscopy) by drawing a spline $15 \mu \mathrm{m}$ wide starting ventrally and extending parallel 961 to the ventricle in the dorsal direction, then using the 'Line profile' or 'Kymograph'

962 function. A $15 \mu \mathrm{m}$ width was chosen as this covered more than a cell width and the 963 average distance a cell travels in $2 \mathrm{hrs}$, the value used for time averaging. 0 distance 964 corresponded to the ventral most end of the spline. Apical, medium and basal 965 expression profiles and kymographs were generated from splines around 10, 30 and $96660 \mu \mathrm{m}$ from the ventricle respectively, and analysing each side of the ventricle 967 separately. 2-3 non-overlapping z-sections were used to generate kymographs per 968 movie. Expression profile data for Draq5 and NGN2 from single snapshot images of 969 live slices were generated in ImageJ using a rectangular ROI of width $15 \mu \mathrm{m}$ and the 970 "Plot profile" function. 
971 Kymographs were analysed using custom scripts in MATLAB. Kymographs were

972 averaged along the time axis in $2 \mathrm{hr}$ windows. Subsequently spatial Venus::HES5

973 intensity in the ventral-dorsal direction was de-trended by fitting a polynomial (order

974 4-6) and subtracting this from the raw data. This removed the larger trend due to the

975 profile of the HES5 expression domain.

976 Auto-correlation and Lomb-Scargle periodograms were used to analyse periodicity of

977 the de-trended spatial intensity plots. Lomb-Scargle periodograms were generated

978 with the MATLAB 'plomb' function and plotting power level thresholds as specified in

979 figure legends. Auto-correlation was performed with the MATLAB 'xcorr' function.

980 Auto-correlation functions were smoothed using Savitzky-Golay filter and then peaks

981 identified using the 'find peaks' function. Significant peaks were identified using a

982 bootstrap method with 100 randomisations. Auto-correlations were randomised and

983 then re-subjected to auto-correlation. 2 standard deviations of the auto-correlations

984 of randomised data was used as a threshold and peaks were designated as

985 significant if they exceeded this threshold. The mean distance between significant

986 peaks was calculated per kymograph timepoint. Fold-changes of spatial intensities

987 were calculated between significant peaks and troughs in the signal identified using

988 'find peaks' on the negative signal.

989 Splitting Venus::HES5 kymographs in to motorneuron and interneuron domains was

990 based on staining of cryo-sectioned E10.5 spinal cord with motorneuron progenitor

991 domain marker OLIG2. The peak of the trend in Venus::HES5 was found to occur on

992 average at $35 \mu \mathrm{m}$ dorsally from the edge of the OLIG2+ domain. This criteria was

993 used to split kymographs from movies of Venus::HES5 spinal cords that had not

994 been immuno-stained.

996 Correlation coefficient analysis in the anterior to posterior (A-P) axis

997 We produced kymographs from multiple non-overlapping stacks extending in the AP 998 direction using the same region of interest (ROI) which meant that Venus:HES5 999 intensity was comparable at the same position in DV. We used detrended 1000 Venus::HES5 averaged over $2 \mathrm{~h}$ per $\mathrm{z}$ and compared the detrended coefficients 1001 pairwise across subsequent z-stacks. Using the confocal magnification in the AP 1002 axis per experiment we reconstructed the absolute distance between subsequent z1003 stacks. Data from untreated and tissue treated with DMSO was analysed in the 1004 same way. 
1006 Hierarchical clustering of local HES5 expression and microcluster persistence

1007 time

1008 Kymographs of HES5 expression were split into adjacent 20 $\mu \mathrm{m}$ regions along the D-

$1009 \mathrm{~V}$ axis and the HES5 intensity averaged in these regions to give a timeseries per

1010 region. To account for any single cell movement in DV, we applied a 2um Gaussian

1011 blur filter onto the kymograph data using the Matlab routine imgaussfilt.m prior to

1012 extracting timeseries per region. These timeseries were normalized to the mean and

1013 standard deviation of each region over time (z-scoring) and subject to hierarchical

1014 clustering using the clustergram, $m$ routine in Matlab with Euclidean distance and

1015 average linkage. The persistence time was calculated as continuous time when the

1016 signal in the region was above (high) or below (low) its mean level. The persistence

1017 ratio was calculated as the time interval spent in a high state divided by the time

1018 interval spent in a low state within the same $20 \mu \mathrm{m}$ region. Where only high or low

1019 persistence time intervals were detected in a region these observations were

1020 excluded from the ratio. We also used an alternative method to compute persistence

1021 time relying on zero-crossing of the detrended Venus::HES5 signal averaged over 0

1022 to $2 \mathrm{~h}$ timepoints; in this approach we identified specific areas containing a

1023 microcluster with high expression (above the mean) and low expression (below the

1024 mean) and repeated the persistence time calculation as described above. For the

1025 calculation of phase synchronisation index see Spatial phase synchronisation.

\section{Phase Mapping and Phase Shift Analysis in Cell Pairs}

1027 We analysed Venus::HES5 ultradian dynamics using the approach in Manning et al.

1028 2019, Phillips et al. 2017. Specifically, we used a Gaussian Processes pipeline to fit

1029 the single cell trend of Venus::HES5 expression (examples shown in Appendix

1030 Figure S2d). We performed detrending of Venus::HES5, followed by z-scoring and

1031 estimated a periodic Ornstein-Uhlenbeck covariance model. This procedure

1032 produces a smooth detrended curve (examples shown in Appendix Figure S4b).

1033 Using the detrended smoothed curves, we extracted the phase shift using cross-

1034 correlation analysis of pairs of timeseries. The pairing was done using Euclidean

1035 distance in the same tissue. The phase shift corresponded to the lag time interval

1036 closest to 0 at which the cross-correlation function shows a peak. From detrended

1037 smooth curves, we then performed Hilbert reconstruction of instantaneous phase 
1038 using the hilbert.m Matlab routine. We used the phase angles corresponding to 1039 neighbouring cell pairs at multiple timepoints to produce a phase mapping. We

1040 plotted the density of the phase map using the dscatter.m routine with $24 \times 24$ binning 1041 of phase values (Eilers and Goeman, Bioinformatics 2004).

\section{Stochastic Multicellular HES5 Model with Time Delay}

1044 The core unit of the multicellular model is a single-cell unit that explicitly models 1045 Hes5 protein and mRNA abundance and is adapted from the work done in Manning 1046 et al., 2019. The single cell model makes use of a Langevin approach to include 1047 stochastic fluctuations in both protein and mRNA as well as the inclusion of a time 1048 delay associated with the inhibitory Hill function used to describe the repressive 1049 action of Hes protein on its own mRNA production. This implementation, along with 1050 the parameter inference (Manning et al., 2019) results in a single cell model capable 1051 of reproducing stochastic oscillations closely matched with the single-cell dynamics 1052 observed in the developing neural tube. The multicellular approach extends the 1053 single cell model by introducing an inhibitory Hill function to couple nearest1054 neighbour cells (in a fixed, no cell movement, hexagonal geometry) whereby high 1055 Hes5 protein in one cell is able to repress Hes5 mRNA production in a neighbouring 1056 cell. This inhibitory Hill function (the coupling function) is representative of the overall 1057 behaviour of the Notch Delta pathway and its interaction with Hes5, allowing for the 1058 bidirectional interaction of Hes5 dynamics between neighbouring cells. Three 1059 parameters are associated with this Hill function that make it flexible enough to 1060 explore different possible coupling realisations of the Notch-Delta pathway, the 1061 effects of which are illustrated in Figure $\mathbf{5 b}$. The main parameter modulated for the 1062 analysis in this paper is the repression threshold which defines the number of protein 1063 molecules that is required to repress mRNA in a neighbouring cell.

\section{Cell to cell HES5 differences by domain and by coupling strength}

1066 We used raw Venus::HES5 data, absolute HES5 quantitation by Fluorescence 1067 Correlation Spectroscopy (FCS) and manually segmented nuclear maps made 1068 available in (Manning et al., 2019). We obtained average HES5 concentration per 1069 nuclei by quantile-quantile matching the Venus distribution to the reference FCS 1070 distribution of HES5 levels across the tissue. Using nuclear centroid location, we 1071 produced absolute cell to cell concentration differences between every cell and its 
1072 closest neighbor. We performed a by domain analysis by dividing the cell to cell

1073 concentration differences by the average HES5 concentration by domain. In the

1074 synthetic examples, HES5 molecular abundance data obtained from the multicellular

1075 model was used to produce absolute cell to cell abundance differences over a range

1076 of coupling strength values. We also produced synthetic cell to cell abundance

1077 differences relative to the mean HES5 abundance per simulation over a range of

1078 coupling strength values.

1079

\section{Phase reconstruction and Kuramoto order value as a measure of synchrony}

1081 To determine the synchronisation of real signals both in the model and experimental 1082 data, the phase of each oscillator was first reconstructed in complex space. This 1083 reconstruction was achieved by using the Hilbert transform, which shifts the phase of 1084 each frequency component in a signal by 90 degrees (Benedetto, 1996). The Hilbert 1085 transform of a function $u(t)$ is defined as

$$
H(u)(t)=\frac{1}{\pi} \int_{-\infty}^{\infty} \frac{u(\tau)}{t-\tau} d \tau
$$

1086 To obtain a rotating vector that contains both the amplitude and phase information of 1087 the signal at a given time $t$, the original signal and the 90 degrees shifted Hilbert 1088 transform can be combined in complex space to give

$$
u_{a}(t)=u(t)+i \cdot H(u)(t) \text {. }
$$

1089 By comparing $u_{a}(t)$ of two or more cells, a measure of how synchronised a 1090 population of cells is can be determined by first calculating what is known as the 1091 complex order parameter

$$
\psi=\frac{1}{N} \sum_{j=1}^{N} e^{i \phi_{j}}
$$

1092 where $\mathrm{N}$ is the number of oscillators and $\phi_{j}$ is the phase of oscillator $j$. From this, the 1093 Kuramoto order parameter is defined as the absolute value of the complex order 1094 parameter $\psi$, which is the magnitude of the vector and has a value between 0 and 1 1095 (Choi et al., 2000). A value of 1 indicates perfect synchrony and matching phase, 1096 meaning that in complex space the phases of each oscillator would be at the same 1097 angle and would rotate at the same frequency. A value of 0 indicates no 1098 synchronisation, and in complex space would appear as a distribution of phases that 1099 average to a point at the origin. 


\section{Phase synchronisation index}

1102 In addition to calculating KOPs, we also used the Hilbert transform to extract phase

1103 from spatial data to determine how dynamic the positions of peak and trough were 1104 over time. This involved extracting and plotting the phase from time-averaged spatial 1105 signals. The phase synchronisation index for DMSO and DBZ conditions (Figure 4d) 1106 was obtained by calculating KOP per position in D-V axis and averaging per z-slice 1107 (with left and right of the ventricle analysed separately).

\section{Statistical testing}

1110 Statistical tests were performed in GraphPad Prism 8. Data was tested for normality

1111 with D'Agostino-Pearson test. The relevant parametric or non-parametric test was 1112 then performed. Bar plots and discrete scatter plots show mean mean \pm SD where 1113 multiple independent experiments are analysed. Statistical significance between 2 1114 datasets was tested with either t-test (parametric) or Mann-Whitney test (non1115 parametric). Statistical significance $(\mathrm{p}<0.05)$ for $2+$ datasets was tested by Kruskall1116 Wallis with Dunn's multiple comparison correction. All tests were 2-sided. Multiple 1117 comparison testing involved comparing all pairs of data columns. Correlations were 1118 analysed using Pearson correlation coefficient. Sample sizes, experiment numbers, $1119 \mathrm{p}$ values<0.05 and correlation coefficients are reported in each figure legend.

\section{Acknowledgements}

1122 We are grateful to members of the Papalopulu lab, Andrew Hazel, James Briscoe 1123 and Andy Oates for advice and discussions. The authors would also like to thank 1124 Robert Lea, the Biological Services Facility and the Bioimaging Facilities of the 1125 University of Manchester for technical support. CM was supported by a Sir Henry 1126 Wellcome Fellowship (103986/Z/14/Z) and University of Manchester Presidential 1127 Fellowship. VB was supported by a Wellcome Trust Senior Research Fellowship to 1128 NP (090868/Z/09/Z). JH, EJ and DH (Wellcome Trust Grant No. 215189/Z/19/Z) 1129 were supported by Wellcome Trust PhD studentships. JK was supported by 1130 Wellcome Trust Senior Research Fellowship to NP and a University of St Andrews 1131 Lectureship. The funders had no role in study design, data collection and analysis, 1132 decision to publish, or preparation of the manuscript.

\section{Author Contributions}


$1135 \mathrm{CM}$ and NP conceived and designed the experimental study.

1136 CM performed wet lab experiments, supervised and performed data analysis,

1137 interpreted data and wrote the paper.

1138 VB supervised and performed development of method to analyse spatial

1139 micropatterns of HES5 expression both in data and from the model, developed

1140 method for Hilbert phase persistence analysis, performed data analysis, interpreted

1141 data and wrote the paper.

$1142 \mathrm{JH}$ designed and implemented stochastic coupled HES5 model, explored

1143 parameterization, analysed and interpreted the model.

1144 XS performed smiFISH and imaging.

1145 EJ collected data and developed method to analyse correlations of HES5 nuclear

1146 intensity.

$1147 \mathrm{DH}$ contributed to the method to analyse periodic spatial micropatterns of HES5

1148 expression.

1149 HB designed and generated Neurog2::mScarlet-I knock-in mouse

1150 ADA designed and generated Neurog2::mScarlet-I knock-in mouse

1151 JK supervised and assisted design, analysis and interpretation of the mathematical

1152 model.

1153 PG supervised and assisted analysis and interpretation of the mathematical model.

1154 NP supervised and directed the work, interpreted data and co-wrote the paper with

1155 CM, VB and JH with input from JK, EJ, DH and PG.

1157 Conflict of interest

1158 None.

\section{Code and Data availability}

1161 All code written in MatLab and is available on GitHub: https://github.com/Papalopulu-

$1162 \underline{\text { Lab/Biga2020 }}$

1163

\section{References}

\section{References}

Bansod S, Kageyama R, Ohtsuka T (2017) Hes5 regulates the transition timing of neurogenesis and gliogenesis in mammalian neocortical development. Development (Cambridge) 144: 3156-3167 
1170 Baron JW, Galla T (2019) Intrinsic noise, Delta-Notch signalling and delayed reactions 1171 promote sustained, coherent, synchronized oscillations in the presomitic mesoderm. $J R$ Soc 1172 Interface 16: 20190436

1173 Benedetto JJ (1996) Harmonic analysis and applications. CRC Press

1174 Boareto M, Jolly MK, Lu M, Onuchic JN, Clementi C, Ben-Jacob E (2015) Jagged-delta 1175 asymmetry in Notch signaling can give rise to a sender/receiver hybrid phenotype. 1176 Proceedings of the National Academy of Sciences of the United States of America 112: 1177 E402-E409

1178 Bonev B, Stanley P, Papalopulu N (2012) MicroRNA-9 Modulates Hes1 ultradian oscillations 1179 by forming a double-negative feedback loop. Cell Rep 2: 10-18

1180 Briscoe J, Small S, 2015. Morphogen rules: Design principles of gradient-mediated embryo patterning. Company of Biologists Ltd, pp. 3996-4009.

Choi MY, Kim HJ, Kim D, Hong H (2000) Synchronization in a system of globally coupled oscillators with time delay. Phys Rev E Stat Phys Plasmas Fluids Relat Interdiscip Topics 61: 371-381

Cohen M, Georgiou M, Stevenson NL, Miodownik M, Baum B (2010) Dynamic Filopodia Transmit Intermittent Delta-Notch Signaling to Drive Pattern Refinement during Lateral Inhibition. Developmental Cell

1190

Corson F, Couturier L, Rouault H, Mazouni K, Schweisguth F (2017) Self-organized Notch dynamics generate stereotyped sensory organ patterns in Drosophila. Science (New York, NY) 356: eaai7407-eaai7407

1191 Das RM, Storey KG (2012) Mitotic spindle orientation can direct cell fate and bias Notch

1192

1193

1194

1195

1196

1197

1198

1199 activity in chick neural tube. EMBO Rep 13: 448-454

Das RM, Storey KG (2014) Apical abscission alters cell polarity and dismantles the primary cilium during neurogenesis. Science 343: 200-204

De Joussineau C, Soulé J, Martin M, Anguille C, Montcourrier P, Alexandre D (2003) Deltapromoted filopodia mediate long-range lateral inhibition in Drosophila. Nature 426: 555-559

de Lichtenberg KH, Funa N, Nakic N, Ferrer J, Zhu Z, Huangfu D, Serup P (2018) GenomeWide Identification of HES1 Target Genes Uncover Novel Roles for HES1 in Pancreatic Development. bioRxiv: 335869

1200

Delile J, Rayon T, Melchionda M, Edwards A, Briscoe J, Sagner A (2019) Single cell transcriptomics reveals spatial and temporal dynamics of gene expression in the developing mouse spinal cord. Development: dev.173807-dev.173807

1203

1204

1205

1206

1207

1208

1209

1210

Galla T (2009) Intrinsic fluctuations in stochastic delay systems: Theoretical description and application to a simple model of gene regulation. Physical Review E 80: 021909-021909

Goodfellow M, Phillips NE, Manning C, Galla T, Papalopulu N (2014) microRNA input into a neural ultradian oscillator controls emergence and timing of alternative cell states. Nat Commun 5: 3399

Hadjivasiliou Z, Moore RE, McIntosh R, Galea GL, Clarke JDW, Alexandre P (2019) Basal Protrusions Mediate Spatiotemporal Patterns of Spinal Neuron Differentiation. Developmental Cell 49: 907-919.e910

1212 Henrique D, Schweisguth F (2019) Mechanisms of Notch signaling: a simple logic deployed in time and space. Development 146

1213 Herrgen L, Ares S, Morelli LG, Schröter C, Jülicher F, Oates AC (2010) Intercellular coupling regulates the period of the segmentation clock. Current Biology 20: 1244-1253

Hunter GL, Hadjivasiliou Z, Bonin H, He L, Perrimon N, Charras G, Baum B (2016) Coordinated control of Notch/Delta signalling and cell cycle progression drives lateral inhibition-mediated tissue patterning. Development (Cambridge, England) 143

Imayoshi I, Isomura A, Harima Y, Kawaguchi K, Kori H, Miyachi H, Fujiwara T, Ishidate F, Kageyama R (2013) Oscillatory control of factors determining multipotency and fate in mouse neural progenitors. Science 342: 1203-1208

1221 Imayoshi I, Kageyama R (2014) bHLH factors in self-renewal, multipotency, and fate choice 1222 of neural progenitor cells. Neuron 82: 9-23 
1223 Isomura A, Ogushi F, Kori H, Kageyama $\mathrm{R}$ (2017) Optogenetic perturbation and

1224 bioluminescence imaging to analyze cell-to-cell transfer of oscillatory information. Genes and

1225 Development 31: 524-535

1226 Jensen MH, Sneppen K, Tiana G (2003) Sustained oscillations and time delays in gene expression of protein Hes1. FEBS Letters 541: 176-177

Kageyama R, Ohtsuka T, Shimojo H, Imayoshi I (2008) Dynamic Notch signaling in neural progenitor cells and a revised view of lateral inhibition. Nature Neuroscience 11: 1247-1251

Kicheva A, Bollenbach T, Ribeiro A, Valle HP, Lovell-Badge R, Episkopou V, Briscoe J (2014) Coordination of progenitor specification and growth in mouse and chick spinal cord. Science (New York, NY) 345: 1254927-1254927

Kobayashi T, Mizuno H, Imayoshi I, Furusawa C, Shirahige K, Kageyama R (2009) The cyclic gene Hes1 contributes to diverse differentiation responses of embryonic stem cells. Genes Dev 23: 1870-1875

Kuzmicz-Kowalska K, Kicheva A (2020) Regulation of size and scale in vertebrate spinal cord development. Wiley Interdiscip Rev Dev Biol: e383

Lewis J (2003) Autoinhibition with transcriptional delay. Current Biology 13: 1398-1408

Ma Q, Chen Z, del Barco Barrantes I, de la Pompa JL, Anderson DJ (1998) neurogenin1 is essential for the determination of neuronal precursors for proximal cranial sensory ganglia. Neuron 20: 469-482

Manning CS, Biga V, Boyd J, Kursawe J, Ymisson B, Spiller DG, Sanderson CM, Galla T, Rattray M, Papalopulu N (2019a) Quantitative single-cell live imaging links HES5 dynamics with cell-state and fate in murine neurogenesis. Nature communications

Manning CS, Biga V, Boyd J, Kursawe J, Ymisson B, Spiller DG, Sanderson CM, Galla T, Rattray M, Papalopulu N (2019b) Quantitative single-cell live imaging links HES5 dynamics with cell-state and fate in murine neurogenesis. Nat Commun 10: 2835

Marklund U, Hansson EM, Sundström E, de Angelis MH, Przemeck GK, Lendahl U, Muhr J, Ericson J (2010) Domain-specific control of neurogenesis achieved through patterned regulation of Notch ligand expression. Development 137: 437-445

Momiji $\mathrm{H}$, Monk NAM (2008) Dissecting the dynamics of the Hes1 genetic oscillator. Journal of Theoretical Biology 254: 784-798

1253 Monk NAM (2003) Oscillatory Expression of Hes1, p53, and NF-kB driven by transcriptional

1254

1255

1256

1257

1258

1259

1260

1261

1262

1263

1264

1265

1266

1267

1268

1269

1270

1271

1272

1273

1274 time delays. Current Biology 13: 1409-1413

Morelli LG, Ares S, Herrgen L, Schröter C, Jülicher F, Oates AC (2009) Delayed coupling theory of vertebrate segmentation. HFSP Journal 3: 55-66

Nelson BR, Hodge RD, Daza RA, Tripathi PP, Arnold SJ, Millen KJ, Hevner RF (2020) Intermediate progenitors support migration of neural stem cells into dentate gyrus outer neurogenic niches. Elife 9

Oates AC (2020) Waiting on the Fringe: cell autonomy and signaling delays in segmentation clocks. Curr Opin Genet Dev 63: 61-70

Ohtsuka T, Ishibashi M, Gradwohl G, Nakanishi S, Guillemot F, Kageyama R (1999) Hes1 and Hes5 as notch effectors in mammalian neuronal differentiation. The EMBO journal 18: 2196-2207

Özbudak EM, Lewis J (2008) Notch signalling synchronizes the zebrafish segmentation clock but is not needed to create somite boundaries. PLoS Genetics 4

Paridaen JT, Huttner WB (2014) Neurogenesis during development of the vertebrate central nervous system. EMBO Rep 15: 351-364

Petrovic J, Formosa-Jordan P, Luna-Escalante JC, Abello G, Iban M, Es, Neves J, Giraldez F, Abelló G, Ibañes M et al (2014) Ligand-dependent Notch signaling strength orchestrates lateral induction and lateral inhibition in the developing inner ear. Development (Cambridge) 141: 2313-2324

Phillips NE, Manning CS, Pettini T, Biga V, Marinopoulou E, Stanley P, Boyd J, Bagnall J, 1275 Paszek P, Spiller DG et al (2016) Stochasticity in the miR-9/Hes1 oscillatory network can account for clonal heterogeneity in the timing of differentiation. Elife 5 
1276 Ramos C, Rocha S, Gaspar C, Henrique D (2010) Two Notch ligands, Dll1 and Jag1, are

differently restricted in their range of action to control neurogenesis in the mammalian spinal cord. PLoS One 5: e15515

Sagner A, Briscoe J, 2019. Establishing neuronal diversity in the spinal cord: A time and a place. Company of Biologists Ltd.

Sagner A, Gaber ZB, Delile J, Kong JH, Rousso DL, Pearson CA, Weicksel SE, Melchionda M, Mousavy Gharavy SN, Briscoe J et al (2018) Olig2 and Hes regulatory dynamics during motor neuron differentiation revealed by single cell transcriptomics. PLOS Biology 16: e2003127-e2003127

Shaya O, Sprinzak D, 2011. From Notch signaling to fine-grained patterning: Modeling meets experiments. pp. 732-739.

Shimojo H, Isomura A, Ohtsuka T, Kori H, Miyachi H, Kageyama R (2016) Oscillatory control of Delta-like1 in cell interactions regulates dynamic gene expression and tissue morphogenesis. Genes \& development 30: 102-116

Shimojo H, Kageyama R, 2016. Oscillatory control of Delta-like1 in somitogenesis and neurogenesis: A unified model for different oscillatory dynamics. Academic Press, pp. 76-82.

Sonnen KF, Lauschke VM, Uraji J, Falk HJ, Petersen Y, Funk MC, Beaupeux M, François P, Merten CA, Aulehla A (2018) Modulation of Phase Shift between Wnt and Notch Signaling Oscillations Controls Mesoderm Segmentation. Cell 172: 1079-1090.e1012

Soto X, Biga V, Kursawe J, Lea R, Doostdar P, Thomas R, Papalopulu N (2020) Dynamic properties of noise and Her6 levels are optimized by miR-9, allowing the decoding of the Her6 oscillator. The EMBO Journal

Sprinzak D, Lakhanpal A, Lebon L, Santat LA, Fontes ME, Anderson GA, Garcia-Ojalvo J, Elowitz MB (2010) Cis-interactions between Notch and Delta generate mutually exclusive signalling states. Nature 465: 86-90

Tiedemann HB, Schneltzer E, Beckers J, Przemeck GKH, Hrabě de Angelis M (2017) Modeling coexistence of oscillation and Delta/Notch-mediated lateral inhibition in pancreas development and neurogenesis. Journal of Theoretical Biology 430: 32-44

Tsiairis CD, Aulehla A (2016) Self-Organization of Embryonic Genetic Oscillators into Spatiotemporal Wave Patterns. Cell 164: 656-667

Vilas-Boas F, Fior R, Swedlow JR, Storey KG, Henrique D (2011) A novel reporter of notch signalling indicates regulated and random Notch activation during vertebrate neurogenesis. BMC Biol 9: 58

Webb AB, Lengyel IM, Jörg DJ, Valentin G, Jülicher F, Morelli LG, Oates AC (2016) Persistence, period and precision of autonomous cellular oscillators from the zebrafish segmentation clock. eLife 5

1314 Figure 1. Microclusters of spinal cord neural progenitor cells have positively 1315 correlated HES5 levels.

1316 (a) Transverse slice of live E10.5 Venus::HES5 homozygous knock-in mouse 1317 showing the ventral HES5 domain in spinal cord ex vivo (left panel); Draq5 live 1318 nuclear stain with nuclear segmentation overlay (right panel); scale bar $30 \mu \mathrm{m}$. (b) 1319 Venus::HES5 nuclear signal corresponding to tissue in (a) obtained by applying 1320 nuclear segmentation onto Venus channel. (c) Pseudo-color look-up table applied to 1321 mean nuclear Venus::HES5 intensity (Materials and Methods) corresponding to 1322 segmented image in (b). Black line surrounding representative microclusters. (d) 
1323 Dimensions of microclusters in cell numbers with high and similar levels of HES5 in i)

1324 apical-basal axes and ii) dorso-ventral at E9.5 (10 microclusters, 3 slices, 3 exps),

1325 E10.5 (10 microclusters, 9 slices, 3 exps) and E11.5 (10 microclusters, 3 slices, 3 1326 exps). NS - no significant difference in one-way ANOVA $p=0.46(A-B), p=0.38(D-V)$.

1327 (e) Pearson correlation coefficient observed in segmented E10.5 homozygous 1328 Venus::HES5 spinal cord ex-vivo slices showing correlation between mean nuclear 1329 Venus::HES5 intensity in any cell compared to its nearest neighbours (see Methods); 1330 dots indicate average per slice; bars indicate mean and standard deviation of 5 1331 slices from 3 experiments (dataset is different from (d).(f) Pearson correlation 1332 coefficient of mean nuclear Venus::HES5 intensity in relationship to distance; red 1333 dots indicate average Venus::HES5 correlation per slice of 12 slices from 3 1334 experiments with corresponding red line indicating polynomial fit (order 2); gray dots 1335 with black line indicate correlations and polynomial fit from 5 randomizations of 1336 intensities analysed in the same way (see Methods). (g) Transverse slice of live

1337 E10.5 Venus::HES5 homozygous knock-in mouse showing the ventral HES5 domain 1338 in spinal cord ex vivo. Scale bar $30 \mu \mathrm{m}$, D- dorsal, V- ventral. (h) Pseudo-color look1339 up table applied to mean nuclear Venus::HES5 intensity of g); centre of intensity 1340 shown with * (i) Pseudo-color look-up table applied to mean nuclear Venus::HES5 1341 intensity in $\mathbf{h}$ ) (only 1 side of ventricle) after radial gradient removal (see methods). 1342 (j) Pearsons correlation coefficient of mean nuclear Venus::HES5 intensity with 1343 distance after subtraction of radial gradient in Venus::HES5 intensity; red dots 1344 represent average in each of 12 slices from 3 experiments.

1346 Figure 2. HES5 microclusters are spatially periodic along dorsal-ventral axis of 1347 spinal cord.

1348 (a) 20x snapshot of an ex-vivo slice culture of E10.5 spinal cord from Venus::HES5 1349 heterozygous knock-in mouse, transverse section; delineated region (blue) 1350 correspond to data shown in (b-c). (b) Spatial profile of Venus::HES5 intensity 1351 averaged over 2.5hrs with 0 distance representing the ventral end of kymograph; 1352 black line represents the trend in Venus::HES5 data across the domain produced 1353 using an polynomial order 6 (see Methods). (c) Detrended spatial profile of 1354 Venus::HES5 corresponding to data shown in (b). (d) Lomb-Scargle Periodogram 1355 analysis of detrended Venus::HES5 data in (c); horizontal line indicates significance 
1356 level $\mathrm{p}=0.0001$; red arrowhead indicate significant peaks. (e) Auto-correlation

1357 analysis of detrended Venus::HES5 spatial profile in (c) with multiple peaks

1358 indicating spatial periodicity; significant peaks (red arrowhead) lie outside gray area

1359 indicating 95\% significance based on bootstrap approach (see Methods) and non-

1360 significant peaks (black arrowhead). (f) Peak to peak distance in auto-correlation

1361 from detrended Venus::HES5 signal collected in apical regions of spinal cord

1362 between E9.5-E11.5; bars indicate mean and SD of individual slices from 3

1363 independent experiments; Kruskal-Wallis test not significant, $p=0.44$. (g)

1364 Representative example of auto-correlation from detrended Draq5 nuclear signal

1365 with peak to peak distances indicative of inter-nuclear distance in live tissue; gray

1366 area denotes 95\% confidence area for Draq5. (h) Peak to peak distance in auto-

1367 correlation of detrended Draq5 spatial profile in apical regions of spinal cord between

1368 E9.5-E11.5; bars indicate mean and SD of individual slices from 3 independent

1369 experiments; Kruskal-Wallis test not significant, $p=0.3$. (i) Schematic of multiple non-

1370 overlapping regions of interest identified as Apical, Intermediate and Basal in the

1371 spinal cord tissue; width of regions in the apical-to-basal direction was $15 \mu \mathrm{m}$. (j)

1372 Peak to peak distance in auto-correlation of detrended Venus::HES5 spatial profile

1373 corresponding to Apical, Intermediate and Basal regions of spinal cord at E10.5;

1374 dataset is different from (h); markers indicate average distance per experiment with

1375 a minimum of 3 z-stacks per experiment and 2 repeats (left and right of ventricle)

1376 analysed per z-stack; bars indicate mean and SD of 6 independent experiments;

1377 Kruskal-Wallis test not significant, $p=0.115$; distances correspond to $4-5$ cells

1378 considering the inter-nuclear distance in DV quantified in (h).

1380 Figure 3. HES5 protein is expressed in a dynamical spatial periodic pattern 1381 modulated by Notch.

1382 (a) Schematic of extracting kymograph information from tissue data by averaging 1383 Venus::HES5 intensities observed in E10.5 heterozygous spinal cord slices to 1384 generate one intensity profile in the dorsal-ventral axis per timepoint (see Methods). 1385 (b) Representative kymograph data showing spatiotemporal Venus::HES5 1386 expression profile along ventral-dorsal direction in a $15 \mu \mathrm{m}$ wide apical region and 1387 observed over $14 \mathrm{~h}$; local bands of $20 \mu \mathrm{m}$ width in $\mathrm{D}-\mathrm{V}$; region of interest markers 1388 indicate: *low to high, ${ }^{* *}$ high to low and ${ }^{* *}$ re-occurring high/low activity in the same 
1389 area. (c) Hierarchical clustering of apical Venus::HE5 expression from one

1390 representative experiment showing behaviour in the same area over time; columns

1391 represent fluctuations in Venus::HES5 intensity in small local areas (bands) obtained

1392 by dividing the spatial signal into non-overlapping $20 \mu \mathrm{m}$ regions and normalizing to

1393 the mean and standard deviation of each region over time (z-scoring); data has been

1394 subject to a Gaussian blur pre-processing step (see Appendix Figure S2b and

1395 Methods). (d) Persistence of Venus::HES5 in $20 \mu \mathrm{m}$ regions expressed as

1396 continuous time intervals when signal in the band is high or low compared to its

1397 mean (see Methods); individual datapoints (gray) indicate quantification of high and

1398 low persistence time obtained from over 300 thin bands collected from multiple

1399 tissues with 2 z-stacks per tissue and 2 repeats (left and right of ventricle) per z-

1400 stack; dots indicate paired medians of 5 independent experiments; statistical test is

1401 paired t test of median per experiment with two tail significance and $p=0.7171$. (e)

1402 Ratio measure indicating time interval at high HES5 over time interval spent in low

1403 HES5 observed in apical $20 \mu \mathrm{m}$ bands; markers indicate ratios collected from over

1404300 bands also shown in (d); black dots indicate the median of 5 separate

1405 experiments and the black line indicates the median of all experiments. (f)

1406 Persistence of Venus::HES5 levels in high and low states taken from tracked single

1407 cells collected from 3 independent experiments; paired $t$ test not significant

$1408 \mathrm{p}=0.0533$. (g) Positional data projected in the DV and AB axis obtained from 3D

1409 tracking of a cell pair showing cells stay in close proximity over 12-15h. (h) Relative

1410 distance between cell pairs computed from relative 3D Euclidean distance between

1411 nuclei over 12-15h; dots indicate median distance over tracking period, horizontal

1412 lines show mean and SD of 14 cell pairs from 3 experiments. (i) Representative

1413 example timeseries of raw Venus::HES5 raw intensity in cells pairs identified as

1414 remaining in close proximity; insert shows Venus::HES5 intensity relative to the

1415 tissue mean intensity; r-values indicate Spearman correlation coefficients between

1416 time traces over all co-existing timepoints. (j) Spearman correlation coefficients

1417 computed in the same cell pairs from Venus::HES5 (Raw), Venus::HES5 relative to

1418 the tissue mean (Rel. to tissue) and H2B:mCherry (control) nuclear intensity

1419 timeseries; dots indicate correlation coefficient of pairs, lines show median from 14

1420 cells from 3 experiments; Kruskal-Wallis test with Dunn multiple comparison with

1421 significance $p<0.001^{* * *}$ and $p<0.0001^{* * *}$. (k) Detrended Venus::HES5 fluorescent 
1422 intensity timeseries (after z-scoring) corresponding to examples in (i); red markers

1423 indicate in-phase peaks. (I) Density phase plots from instantaneous Hilbert phase

1424 reconstruction at multiple timepoints over a 12-14h period; dots indicate the phase

1425 angle in Cell 1 and Cell 2 from 14 pairs collected from 3 experiments; colormap

1426 indicates probability density showing accumulation of phase values predominantly

1427 along the $(0,0)$ and $(2 \pi, 2 \pi)$ diagonal; light colours indicate most frequent.

1429 Figure 4. Notch inhibition increases HES5 pattern persistence.

1430 (a) Start:Finish Venus::HES5 intensity ratio in E10.5 Venus::HES5 heterozygous

1431 spinal cord slices treated with control (DMSO) and Notch inhibitor DBZ (2 $\mu M)$

1432 observed over 16h; bars indicate mean and standard deviation of DMSO ( $\mathrm{n}=3$

1433 experiments) and DBZ ( $n=4$ experiments); 2-tailed t-test $p=0.0001$. (b)

1434 Representative spatiotemporal plots of the detrended Venus::HES5 pattern along

1435 ventral-dorsal direction in (i) DMSO control and (ii) DBZ conditions obtained by

1436 averaging kymographs data in the same region over $2 \mathrm{hr}$ time intervals. (c-i)

1437 Schematic indicating the correspondence between Venus::HES5 spatial oscillator

1438 represented as detrended level and phase angle characteristics; the spatial oscillator

1439 traverses repeated cycles including start (HES5 low-orange arrowhead), middle

1440 (HES5-teal arrowhead) and end (HES5 low-red arrowhead) which in phase space

1441 corresponds to phase angles $0, \pi$ and $2 \pi$ respectively. (c-ii,iii) Phase maps

1442 corresponding to (ii) DMSO and (iii) DBZ detrended Venus::HES5 data shown in (b-

1443 i) and (b-ii) respectively. (d) Phase synchronisation measure (see Methods) of the

1444 detrended Venus::HES5 spatial oscillator over time in E10.5 Venus::HES5 spinal

1445 cord slices treated in DMSO vs DBZ conditions; dots indicate DMSO (21

1446 kymographs, $\mathrm{n}=3$ experiments) and DBZ (19 kymographs, $\mathrm{n}=4$ experiments); 2 tailed

1447 Mann-Whitney test $p=0.0207^{*}$. (e) Spatial peak: trough fold change in Venus::HES5

1448 intensity profile in the D-V axis measured at $2 \mathrm{~h}$ and 10h in DMSO and DBZ treated

1449 E10.5 Venus::HES5 spinal cord slices; dots indicate average over 3 z-slices from

1450 DMSO ( $n=3)$ and DBZ ( $n=4)$ experiments; lines indicate median per condition; 1

1451 tailed unpaired $t$ test with significance $p<0.05^{\star}$. (f) Percentage of ex-vivo slices with

1452 significant spatial period detected after 10-12hrs of DMSO and DBZ conditions;

1453 significant spatial period defined as multiple significant peaks in auto-correlation

1454 detected above the 95\% confidence bounds; dots indicate \% per experiment; bars 
1455 denote median and inter-quartile range of DMSO $(n=3)$ and DBZ $(n=4)$ experiments;

14561 tailed $t$ test with significance $p=0.0062^{\star *}$. (d) Peak:to:peak distance in auto-

1457 correlation plots of detrended Venus::HES5 spatial profile in DMSO and DBZ treated

1458 E10.5 Venus::HES5 spinal cord slices; gray dots represent significant mean peak-to-

1459 peak distance of DMSO (100) and DBZ (105) auto-correlation functions collected

1460 from 3 z-stacks per slice and 2 repeats (left and right of ventricle) with multiple

1461 timepoints; bars indicate median per experiments from DMSO $(n=3)$ and DBZ $(n=4)$

1462 experiments; 2 tailed Mann-Whitney test $p<0.0001$.

1464 Figure 5. Multicellular cell-cell coupling model explains the emergence of 1465 microclusters.

1466 (a) Schematic of repressive interactions via Notch-Delta between neighbouring cells 1467 whereby the effects of HES5 protein in Cell 1 (marked as $P_{1}$ ) on transcription in Cell

14682 and vice-versa are represented using an inter-cellular Hill function $J\left(P_{1,2}\left(t-\tau_{N D}\right)\right)$

1469 where $t$ denotes time and $\tau_{N D}$ represents the intercellular time delay, the time 1470 interval required to synthesise the intermediate molecular species (detailed in Figure 1471 EV4a); HES5 auto-repression is represented using an intra-cellular Hill function $1472 G\left(P_{1,2}\left(t-\tau_{H}\right)\right)$ where $\tau_{H}$ represents the inter-cellular time delay, the time interval 1473 required for protein to be produced and repress its own transcription. (b) 1474 Mathematical description of the inter-cellular Hill function and its parameters: time 1475 delay $\left(\tau_{N D}\right)$, repression threshold $\left(P_{0}\right)$ and Hill coefficient $(n)$; (bottom left panel) 1476 higher $P_{0}$ corresponds to reduced inter-cellular repression (i.e. decreased coupling 1477 strength) and conversely lower $P_{0}$ corresponds to higher coupling strength; (bottom 1478 right panel) increasing values of $n$ correspond to increased steepness of the inter1479 cellular response. (c) Multiscale coupled mathematical model of the tissue 1480 environment consisting of a 2D hexagonal grid of cells expressing HES5 protein with 1481 corresponding auto-repression (described in (a)) coupled together by repressive 1482 interactions between its 6 immediate neighbours (see Methods); single cell inter1483 cellular repression is a Hill function (with parameters described in (b)) dependent on 1484 mean protein abundance in the neighbouring cells. (d) Parameter exploration of 1485 single cell temporal period emerging from the model at different repression threshold 1486 and time delay values. (e) Parameter exploration of phase synchronisation quantified 1487 using the Kuramoto Order Parameter (see Methods) where 1 indicates global in- 
1488 phase activity and 0 indicates no co-ordination of phase between cells. (f) Parameter

1489 selection strategy combining experimentally determined temporal phase (insert left 1490 panel) and KOP (insert right panel) values in spinal cord tissue (see (Manning et al., 1491 2019a) and Methods) to indicate areas where model statistics (i.e. mean temporal 1492 period and KOP of synthetic data) resemble real tissue; values within $\pm 1 S D$ and $14932 \pm S D$ from the mean of the tissue are identified and values found outside of $\pm 2.4 S D$ 1494 from the mean of tissue are excluded. (g) Representative examples of synthetic 1495 kymograph data obtained at specific levels of repression threshold: Alternating high1496 low $\left(P_{0}=400\right)$, Global in phase $\left(P_{0}=15000\right)$ and Local in phase $\left(P_{0}=21000\right)$ and 1497 corresponding KOP values; the presence of microclusters at weak coupling is 1498 indicated with red arrowheads; time delay 150min, $n=4$. (h) Kymograph data 1499 obtained in the absence of coupling between cells; phase relationships are un1500 coordinated resulting in a KOP $\approx 0$. (i-j) Synthetic data timeseries corresponding to 1501 simulations in $(\mathbf{g})$.

1502

1503 Figure 6. Cell-cell coupling strength can regulate probability of differentiation 1504 in a multicellular environment.

1505 (a) Representative synthetic timeseries example and mathematical description of 1506 probability of differentiation $\left(P_{\text {diff }}\right)$ in relation to population mean HES5 protein levels 1507 (referred to as 'differentiation threshold', $D_{\text {thresh) }}$ whereby HES5 protein abundance $1508(P(t))$ dropping below the threshold increases the rate at which cells differentiate. (b) 1509 Differentiation rates estimated from the multicellular coupled model (detailed in 1510 Figure 5) over a wide range of repression threshold values corresponding to 1511 decreasing coupling strength; three dynamic regimes are labelled as Alternating 1512 high-low, Global in phase and Local in phase mirroring examples shown in Figure 1513 5g,i. (c) Analysis of temporal CoV and spatial CoV from synthetic data 1514 corresponding to differentiation rates shown in (b); these statistics indicate that 1515 spatial variability correlates better with differentiation rates meanwhile temporal 1516 variability shows only a moderate quasi- linear increase in Alternating high-low 1517 conditions compared to the rest. Single cell parameters used to generate (b) and (c) 1518 are shown in Appendix Table S3-Main, and the multicellular parameters used were $1519 n_{N D}=4, \tau_{N D}=150$ minutes. Each value plotted in (b) and (c) shows the mean and 1520 SD from 10 simulations at each repression threshold value. 
1522 Figure 7. Type of HES5 spatial pattern and coupling strength correlates with 1523 rate of differentiation in motorneuron and ventral interneuron domains.

1524 (a) Transverse cryosection of E10.5 Venus::HES5 spinal cord. Venus::HES5 1525 endogenous signal, OLIG2 - motorneuron progenitor marker, NGN2 - early marker 1526 of neuronal commitment; scale bar $20 \mu \mathrm{m}$. (b) Relative nuclear intensities of 1527 Venus::HES5 and NGN2 in motorneuron and interneuron progenitors; bars show 1528 mean and SD of at least 494 cells per domain from 5 slices in 2 experiments; 1529 Kruskal-Wallis with Dunn's multiple comparison test adjusted $p$-values ${ }^{* *} p=0.0032$, $1530{ }^{* * *} p<0.001$. (c) Pseudo-color look-up table applied to mean nuclear Venus::HES5 1531 intensity within motorneuron (MN) and interneuron (IN) domains, corresponding to 1532 segmented image in (a). (d) Dimension of microclusters in DV axis for MN and IN 1533 domains; microclusters counted contained cells with high and similar levels of HES5 1534 (Methods); data consists of 34 microclusters measured from 5 sections and 3 1535 independent experiments; 2-tailed Mann-Whitney test. Exact $p$-values ${ }^{* * *} p<0.0001$.

1536 (e) Peak to peak distance in auto-correlation plots of de-trended Venus::HES5 1537 spatial profile in MN and IN domains; this is a measure of spatial period in 1538 Venus::HES5 profile along dorsal-ventral axis of spinal cord; grey data points 1539 represent mean peak to peak distance of at least 3 slices with left and right ventricle 1540 analysed separately in 6 experiments; black dots show median per experiment and 1541 line shows overall median; 2-tailed Mann-Whitney test $p$-values ${ }^{* * * *} p<0.00001$. (f) 1542 Cell-cell concentration differences in HES5 between neighbours, normalised to mean 1543 concentration of HES5 in that domain; grey data points represent normalised 1544 concentration difference between a pair of neighbours, bars shows mean and SD; 2 1545 independent experiments; 2-tailed Mann-Whitney test with $p$-values ${ }^{* \star *} p=0.003$, $1546{ }^{* * * *} \mathrm{p}<0.00001$.

1548 Figure 8. NGN2 expression is spatially periodic and positively correlates with 1549 the HES5 pattern.

1550 (a) Detrended spatial profile of NGN2::mScarlet-I intensity from transverse slice of 1551 E10.5 spinal cord from heterozygous knock-in mouse in ventral-dorsal direction;red 1552 indicates motorneuron (MN) domain, blue interneuron domain (IN). (b) Auto1553 correlation analysis of detrended NGN2::mScarlet-I intensity spatial profiles from 1554 motorneuron and interneuron domains; multiple peaks indicating spatial periodicity; 
1555 significant peaks (red triangle) lie outside black dotted lines indicating 95\% 1556 significance based on bootstrap approach (see Methods) and non-significant peaks 1557 (black triangle). (c) Peak to peak distance in auto-correlation plots of de-trended 1558 NGN2::mScarlet-I spatial profile in motorneuron (MN) and interneuron (IN) domains 1559 as a measure of spatial period in NGN2 expression along dorsal-ventral axis of 1560 spinal cord; Grey data points represent mean peak to peak distance in a single slice, $1561 \mathrm{n}=33$, left and right ventricle analysed separately in 4 experiments; black line shows 1562 overall mean, error bars show SD; 2-tailed Mann-Whitney test with exact p-value *** $1563 \mathrm{p}=0.0003$. (d) Ratio of NGN2:HES5 spatial period in the same tissue; grey dots show 1564 ratio for single image from 4 experiments; line shows overall median and error bars $156595 \%$ confidence limits. (e) Detrended spatial profile of Venus::HES5 (black) and 1566 NGN2::mScarlet-I (red) intensity from the same transverse slice of E10.5 spinal cord 1567 in ventral-dorsal direction. (f) Example cross-correlation function of Venus::HES5 1568 with NGN2::mScarlet-I (blue), Venus::HES5 with brightfield signal (black), and 1569 NGN2::mScarlet-I with brightfield signal (red) from the same transverse slice of 1570 E10.5 spinal cord; markers indicate the presence of two types of co-ordination 1571 namely in-phase (red arrowhead) and out-of-phase (black arrowhead). (g) Phase 1572 shift showing absolute lag distance corresponding to in-phase peak in Venus::HES5 1573 vs NGN2::mScarlet-I cross-correlation function of spatial intensity profiles from the 1574 same slice; red line indicates average inter-nuclear distance in D-V; 2-tailed Mann1575 Whitney test not significant, $p=0.32$. (h) Pseudo-color look-up tables applied to mean 1576 nuclear Venus::HES5 and NGN2 staining intensity in motorneuron (MN) and 1577 interneuron (IN) domains. Venus::HES5 microcluster and single NGN2 high cell (red 1578 arrow) in IN domain; Alternating high-low expression of Venus::HES5 in MN, red 1579 arrows show high cells. (i) Graphical summary: Through a combination of 1580 experimental and computational work we characterised the HES5 dynamic 1581 expression in the mouse E10.5 ventral spinal cord. We found evidence that 1582 progenitors located in two domains (motorneuron, MN and interneuron, IN) give rise 1583 to distinct spatiotemporal characteristics that are indicative of differences in coupling 1584 strength and can explain increased differentiation rates observed in MN. 
1588 Figure EV1. Venus::HES5 expression in single progenitor cells located in the 1589 ventral domain of spinal cord. Related to Figure 1.

1590 (a) Transverse slice of live Venus::HES5 homozygous knock-in mouse spinal cord

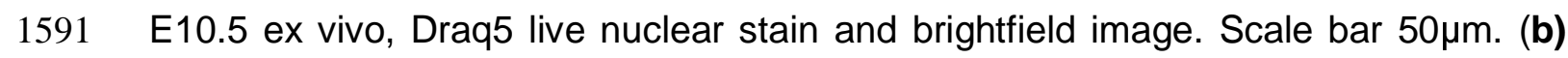
1592 Immunofluorescence of E10.5 Venus::HES5 transverse slice of spinal cord ex-vivo. 1593 SOX2+ progenitors and endogenous Venus::HES5 signal. Scale bar $40 \mu \mathrm{m}$. (c) 1594 Viridis look-up table applied to mean nuclear Draq5 intensity corresponding to 1595 transverse slice in Figure 1c. (d) Pearson correlation coefficient of mean nuclear 1596 Venus::HES5 intensity in relationship to distance for (i) E9.5 and (ii) E11.5 1597 Venus::HES5 spinal cord ex-vivo slices; red dots indicate average Venus::HES5 1598 correlation per slice of 3 slices from 3 experiments with corresponding red line 1599 indicating one phase decay fit. Black line denotes 95\% confidence levels. Gray dots 1600 indicate correlations from randomizations of intensities analysed in the same way.

1601 (e) Transverse slice of live (i) Venus::HES5 homozygous knock-in mouse spinal cord 1602 E10.5 ex vivo showing (ii) segmentation of Draq5 and (iii) mask applied to 1603 Venus::HES5 channel. Images correspond to slice shown in Figure 1g. (f) 1604 Representative example of mean Venus::HES5 intensity as a function of radial 1605 distance with respect to center of intensity (see Methods). (g) and (h) Left panels 1606 Viridis look-up table applied to mean nuclear Venus::HES5 intensity in E9.5 and 1607 E11.5 slices respectively after radial gradient removal. Right panels - Pearson 1608 correlation coefficient of mean nuclear Venus::HES5 intensity in relationship to 1609 distance for E9.5 and E11.5 Venus::HES5 spinal cord ex-vivo slices respectively. 1610 Red dots indicate average Venus::HES5 correlation per slice of 3 slices from 3 1611 experiments with corresponding red line indicating one phase decay fit. Black line 1612 denotes 95\% confidence levels. Gray dots indicate correlations from randomizations 1613 of intensities analysed in the same way.

1615 Figure EV2. Draq5 and Venus::HES5 spatial periodicity in live spinal cord 1616 tissue slices. Related to Figure 2.

1617 (a) Transverse slice of live Venus::HES5 homozygous knock-in mouse spinal cord 1618 E10.5 ex vivo, Draq5 live nuclear stain; rectangle shows region for spatial profile. (b) 1619 Viridis look-up table applied to mean nuclear Venus::HES5 intensity in the same 1620 slice shown in (a) and (b), rectangle shows the same region of interest for spatial 1621 profile. (c) Venus::HES5 intensity spatial profile (black) from the yellow box in (a), 
1622 and trend line in blue (fitted polynomial order 6). (d) Detrended spatial profile of 1623 Venus::HES5 (grey) and Draq5 (red) nuclear stain from region delineated in (a); 1624 arrows show regions of Venus::HES5 low and Draq5 high indicating low 1625 Venus::HES5 areas are not nuclei free. (e) Distribution of peak-to-peak distance in 1626 auto-correlation plots of Draq5 spatial profile; this is a measure of inter-nuclear 1627 distance in Draq5 profile along dorsal-ventral axis of spinal cord; data points 1628 represent all peak to peak distances from 9 slices, 6 experiments; mean $9.3 \pm 0.42$ $1629 \mu \mathrm{m}$ (95\% confidence limits). (f) Spatial periodicity detected by Lomb-Scargle 1630 periodogram in apical, medium and basal regions (10, 30 and $60 \mu \mathrm{m}$ from ventricle 1631 respectively); dots indicate mean periodicity from at least 3 z-sections and both left 1632 and right sides of ventricle analysed in 6 experiments; lines indicate mean and SD 1633 per experiment; Kruskal-Wallis test not significant, $p=0.3137$. (g) Longitudinal cryo1634 section of neural tube in E10.5 Venus::HES5 embryos. A-anterior, P-posterior. Scale 1635 bar $60 \mu \mathrm{m}$. (h) Representative detrended spatial profile of Venus::HES5 from neural 1636 tube in anterior-posterior (A-P) direction. (i) Representative auto-correlation of 1637 Venus::HES5 spatial profile in A-P direction. Multiple significant peaks indicate 1638 spatial periodicity in A-P direction. Significant peaks (red triangle) lie outside black 1639 dotted lines indicating 95\% significance based on bootstrap approach (see Methods) 1640 and non-significant peaks (black triangles). (j) Pearson correlation coefficient of 1641 detrended Venus::HES5 spatial profile between subsequent z-sections of transverse 1642 E10.5 spinal cord tissue slices at known distances (i.e correlations in A-P direction); 1643 untreated slices - dots show 18 pairs of z-sections from 6 experiment; DMSO 1644 treated slices - dots show 9 pairs from 3 experiments. (k) (Left panel)

1645 Representative spatiotemporal plot of the detrended Venus::HES5 pattern along 1646 ventral-dorsal direction ( 0 to $200 \mu \mathrm{m}$ ) obtained by averaging kymograph data in the 1647 same region over 2 hr time intervals; (Right panel) Representative phase map of 1648 spatially periodic Venus::HES5 intensity obtained using the Hilbert transform (see 1649 Methods) from data shown in the left panel; markers indicate areas underdoing a 1650 high-to-low * and low-to-high** transition.

1652 Figure EV3. Changes in Venus::HES5 spatiotemporal expression pattern in live 1653 slice cultures treated with Notch inhibitor DBZ. Related to Figure 4. 
1654 (a) Representative examples of the detrended Venus::HES5 signal observed along 1655 ventral-dorsal direction in DMSO and DBZ conditions; Venus::HES5 intensity data 1656 obtained by averaging kymographs over 2 hrs intervals; panels represent individual 1657 slice cultures (2 per condition) in addition to examples in Figure 4b. (b) Spatial 1658 phase maps obtained from detrended Venus::HES5 signal in DMSO and DBZ 1659 conditions; panels correspond to Venus::HES5 intensity traces shown in (a). (c) 1660 Auto-correlation analysis of detrended apical Venus::HES5 spatial profile in DMSO 1661 (control) and DBZ $(2 \mu \mathrm{M})$ treated E10.5 Venus::HES5 spinal cord slices; panels 1662 show auto-correlation of detrended Venus::HES5 signal averaged from 0-2h of 1663 timelapse video (top panels) and corresponding auto-correlation functions in the 1664 same slices averaged in $2 \mathrm{hr}$ windows for 10h; we observed a decrease in amplitude 1665 of auto-correlation peaks over time in DBZ-treated slices. (d) Peak to peak distance 1666 in auto-correlation from spatial data shown in Figure $\mathbf{4 g}$ presented as median per 1667 experiment from DMSO ( $n=3$ experiments) and DBZ ( $n=4$ experiments); 1 tailed $t$ 1668 test $p=0.0526$. (c) Nuclear density represented by the $\%$ area covered by nuclei in 1669 DMSO and DBZ treated ex-vivo E10.5 spinal cord slice cultures dots indicate 1670 multiple z-stacks from DBZ (5 slices) and DMSO (4 slices); bars indicate mean and 1671 SD per condition; 2 tailed t test $p=0.0004^{\star \star *}$.

1672 Figure EV4. HES5 spatiotemporal dynamics correlate with rate of 1673 differentiation.

1674 (a) Diagram of Notch-Delta inter-cellular communication and HES5 interactions. Our 1675 model takes into account that HES5 negatively regulates its own mRNA production 1676 (gray highlighted area), downstream proneural genes and Delta; the direct and/or 1677 indirect (via proneural genes) repression of Delta by HES5 is expressed 1678 mathematically through the means of an inter-cellular Hill function (see Methods). (b) 1679 Transverse cryosection of E10.5 Venus::HES5 spinal cord. Venus::HES5 1680 endogenous signal, OLIG2: motorneuron progenitor marker, NGN2: early marker of 1681 neuronal commitment, DAPI; scale bar $25 \mu \mathrm{m}$. (c) Spatial expression profile of 1682 Venus::HES5, NGN2 and OLIG2 from the same tissue to help delineate 1683 motorneuron (OLIG2+) vs interneuron (OLIG2-) domains. (d) Spatial profile of 1684 Venus::HES5 intensity (black) generated by averaging 2.5hrs of kymograph data; 0 1685 distance represents ventral end of kymograph; blue dotted line is trend in 1686 Venus::HES5 data across the domain determined by polynomial fit order 6; domain 1687 boundary between motorneuron progenitors (MN) and interneuron progenitors (IN) 
1688 marked with red dashed line. (e) Detrended spatial profile of Venus::HES5 1689 corresponding to (c) in motorneuron progenitors (red:MN) and interneuron 1690 progenitors (blue:IN). (f) Auto-correlation plot of detrended Venus::HES5 spatial 1691 profile in MN and IN progenitors; black lines show confidence limits for peak 1692 significance based on bootstrap approach on de-trended Venus::HES5 intensity 1693 profile (see Methods); red triangle - significant peak, black triangle - non-significant 1694 peak; multiple significant peaks in auto-correlation shows periodicity in spatial profile 1695 of Venus::HES5 intensity. (g) Spatial periodicity in motorneuron vs interneuron 1696 domain measured with the Lomb-Scargle periodogram; top 2 significant peaks were 1697 used to calculate spatial period from 2 to 3 z-sections per experiment, left and right 1698 side of ventricle analysed separately and 6 experiments; bars indicate mean with 1699 SD; Mann-Whitney test with two-tail significance for $\mathrm{p}<0.0001^{* * *}$. (h) Fold-change in 1700 Venus::HES5 spatial pattern between hi-low regions in IN domain relative to MN 1701 domain. Data points represent mean per experiment. Lines shows mean and SD of 6 1702 experiments. 2-tailed Mann-Whitney test $\mathrm{p}=0.0022^{* *}$.

1704 Figure EV5. Exploration of HES5 spatiotemporal dynamics in a multicellular 1705 environment.

1706 (a) Schematic of Hes5, DIl1 and Jag1 expression in progenitor domains along D-V

1707 axis of E10.5 mouse spinal cord compiled based on data from (Manning et al., 1708 2019b) and (Marklund et al., 2010). (b) DAPI nuclear stain of interneuron region in 1709 E10.5 mouse; lines indicate spatial localisation in tissue. (c) Single-molecule FISH of 1710 DII1 and Hes5 expression in spinal cord region corresponding to (b) (Materials and 1711 Methods); panels 1 and 2 indicate DIl1 and Hes5 respectively; panels 3 and 4 show 1712 false-color merged DII1/Hes5 and DII1/Hes5/DAPI respectively; scale bar $20 \mu \mathrm{m}$. 


\section{Figure 1.Microclusters of cells have positively correlated HES5 $\mathrm{H}$. a}

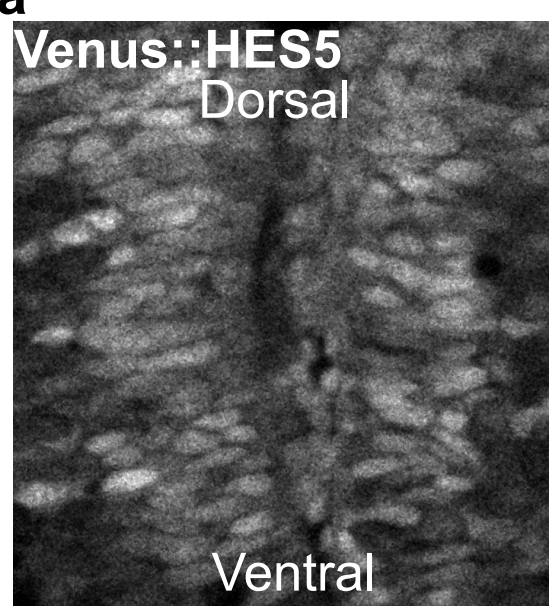

C

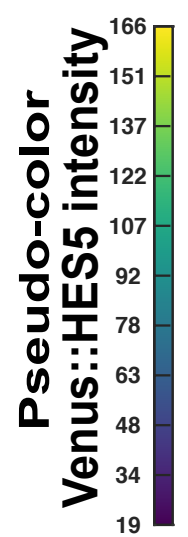

e

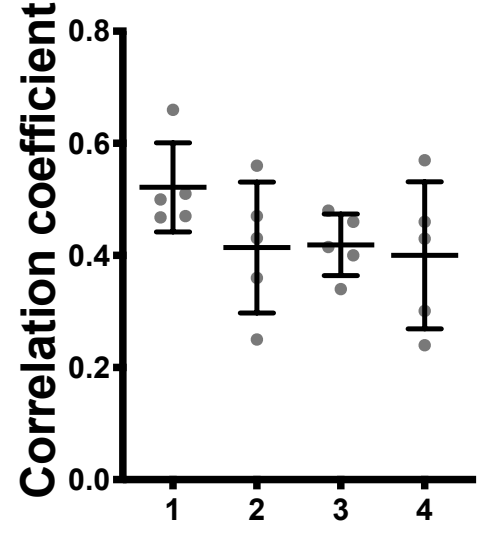

No. of neighbours

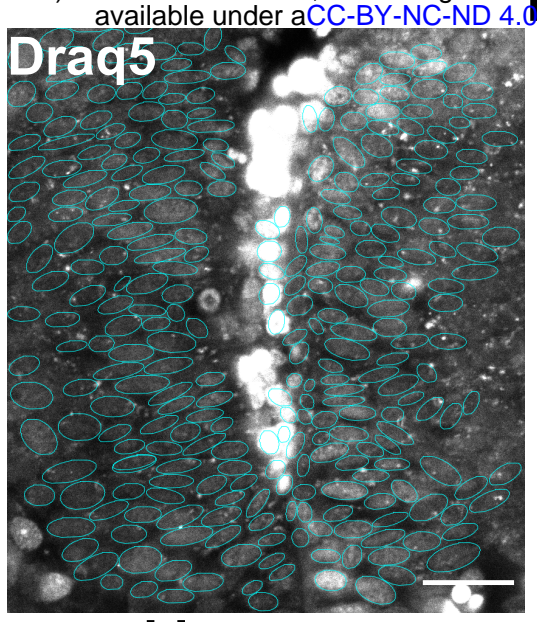

d i

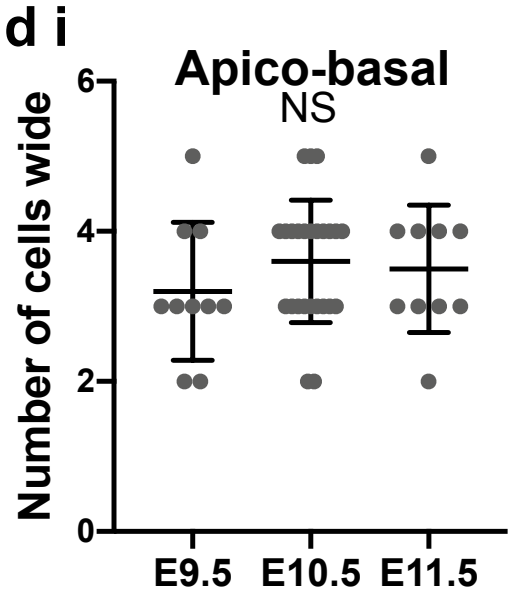

f

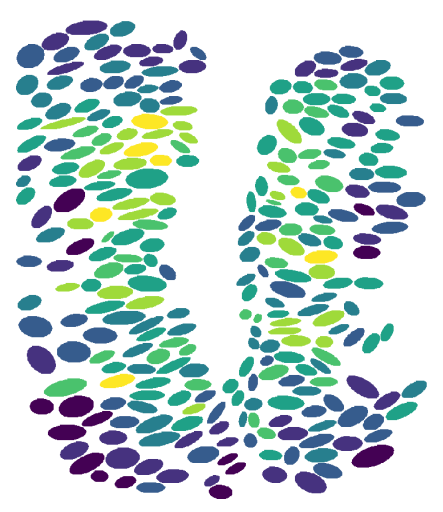

h
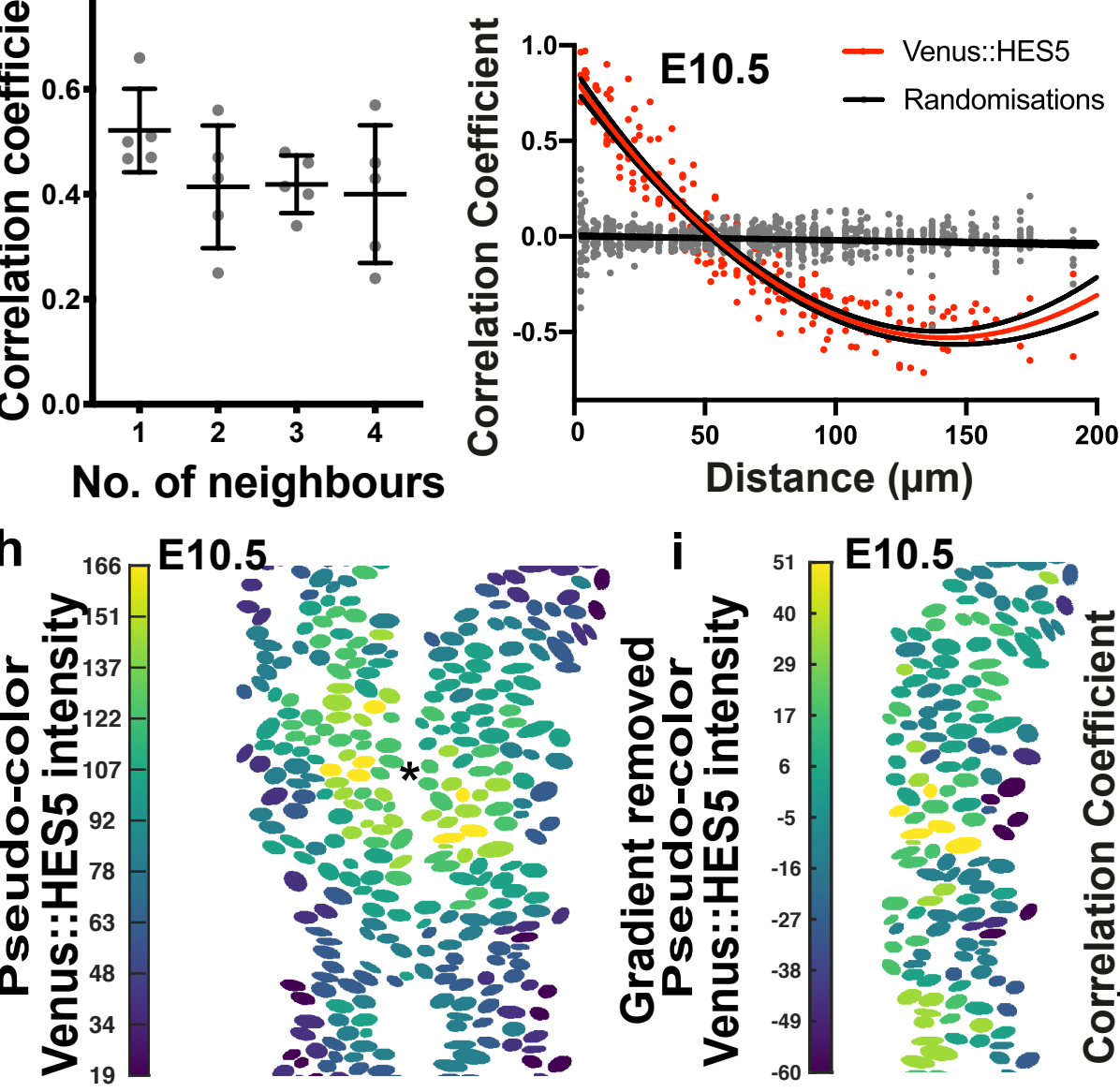

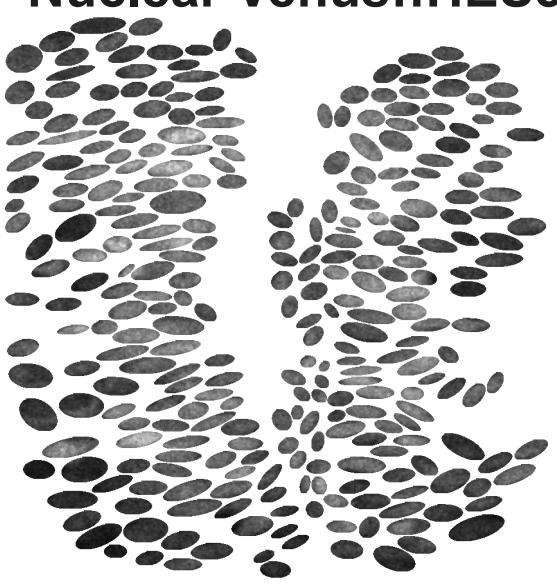

ii

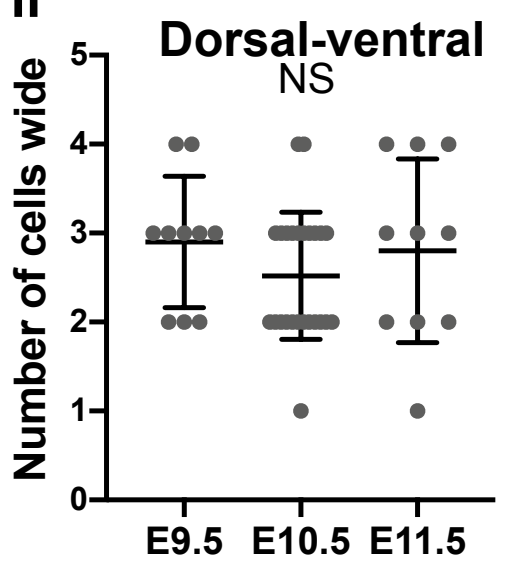

g

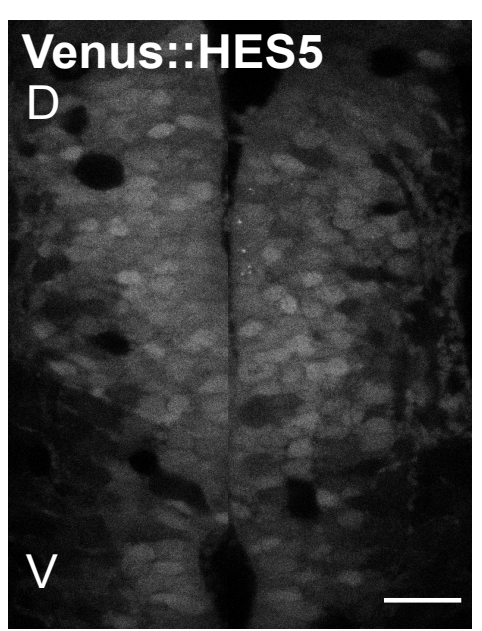

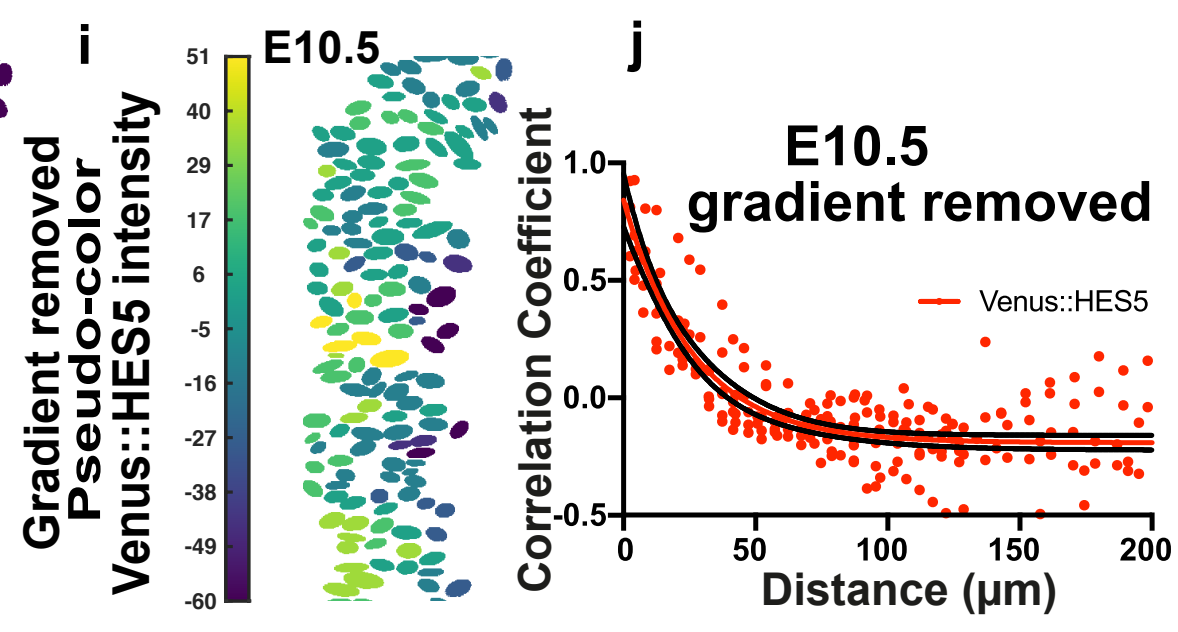


Figure 2. HES5 micro-clusters are spatially periodic along dorsal-inentral axis of spinal cord

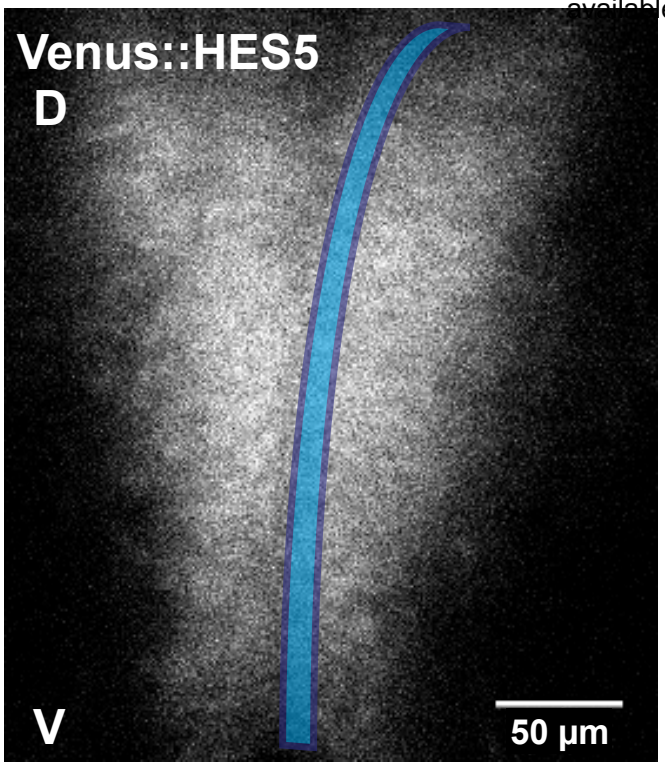

$\underset{15 \mu \mathrm{m}}{\longleftrightarrow}$

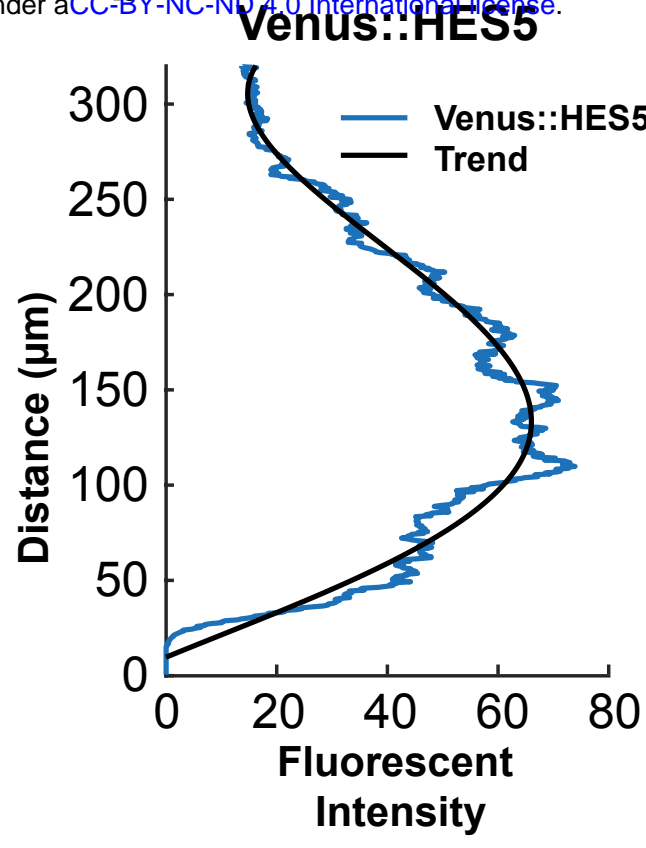

Venus::HES5

d

e $\times 10^{3}$
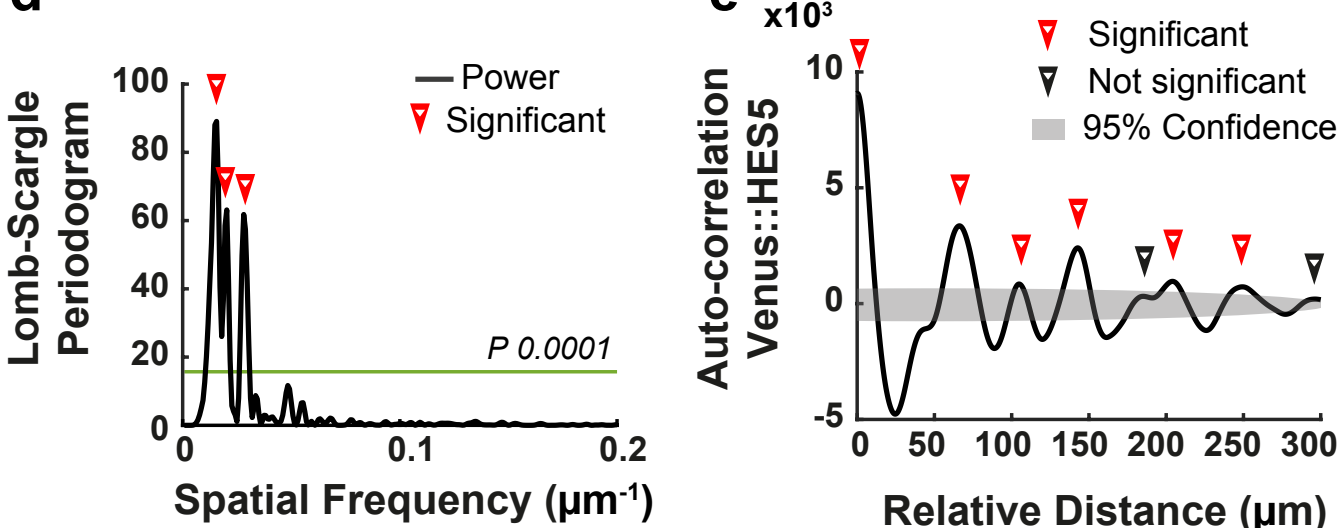

Relative Distance $(\mu \mathrm{m})$

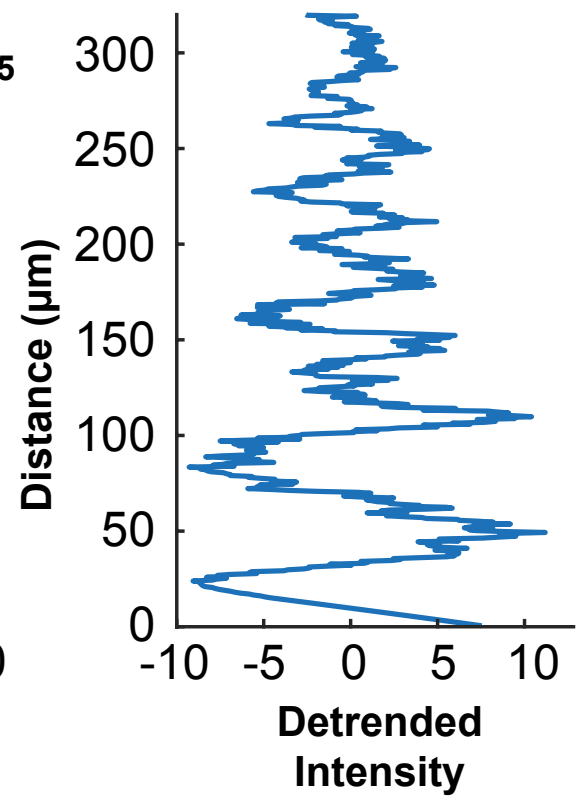

9

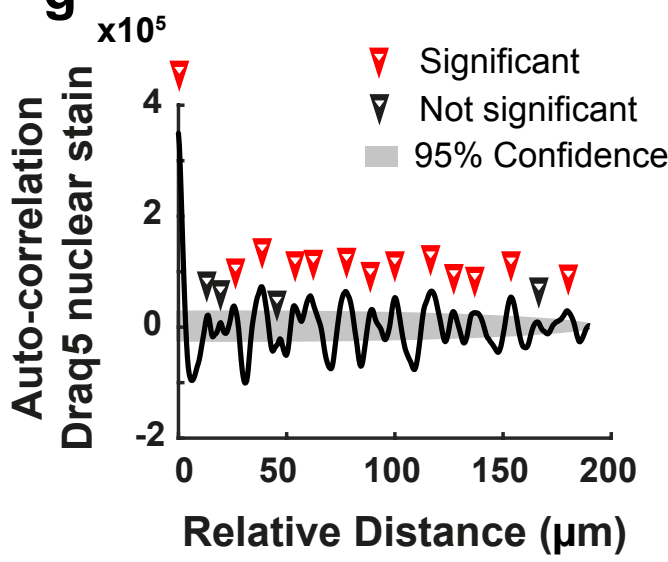

h
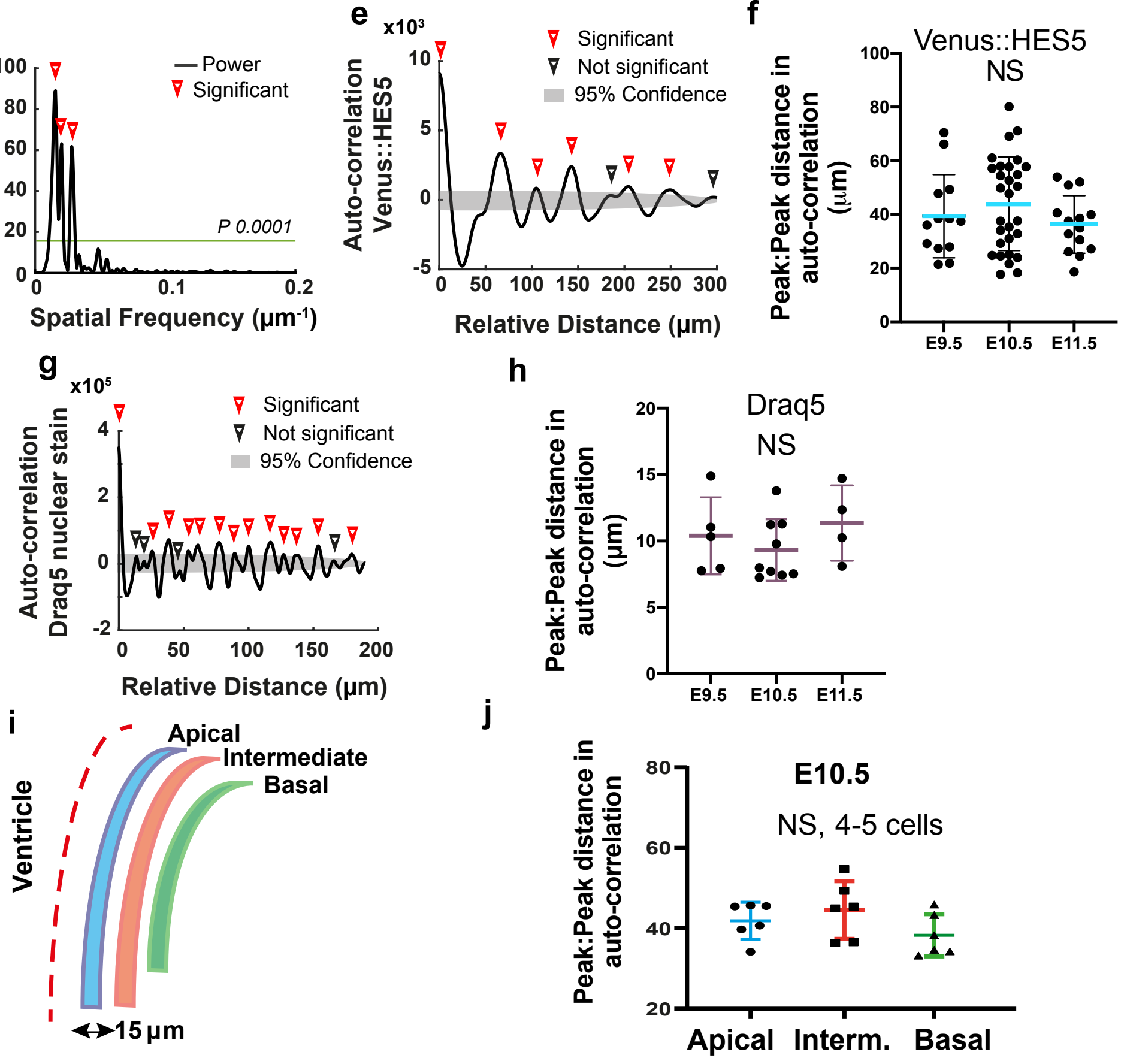
bi iopth preprint doi: https://doi.org/10.1101/2020.08.03.234369; this version posted December 13, 2020. The copyright holder for this preprint (which was not certified by peer review) is the author/funder, who has granted bioRxiv a CAlustelinithg thofreprint in perpetuity. It is made

a

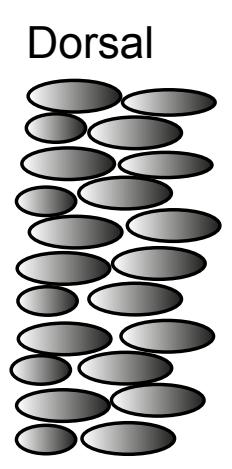

Ventral

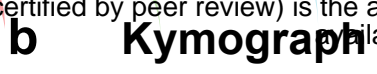

b
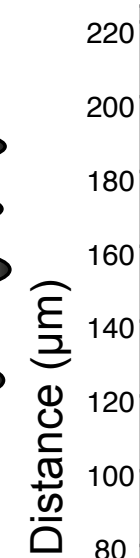

20

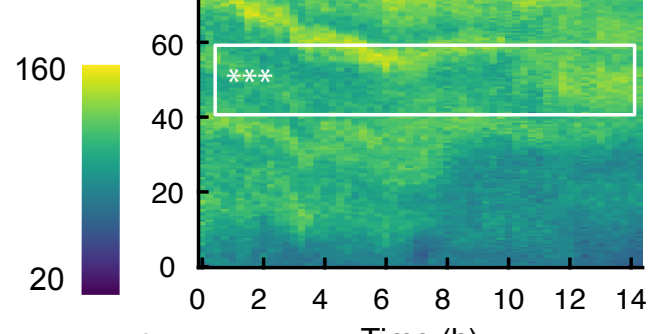

Venus::HES5

Intensity

$\mathbf{f}$

Single Cells
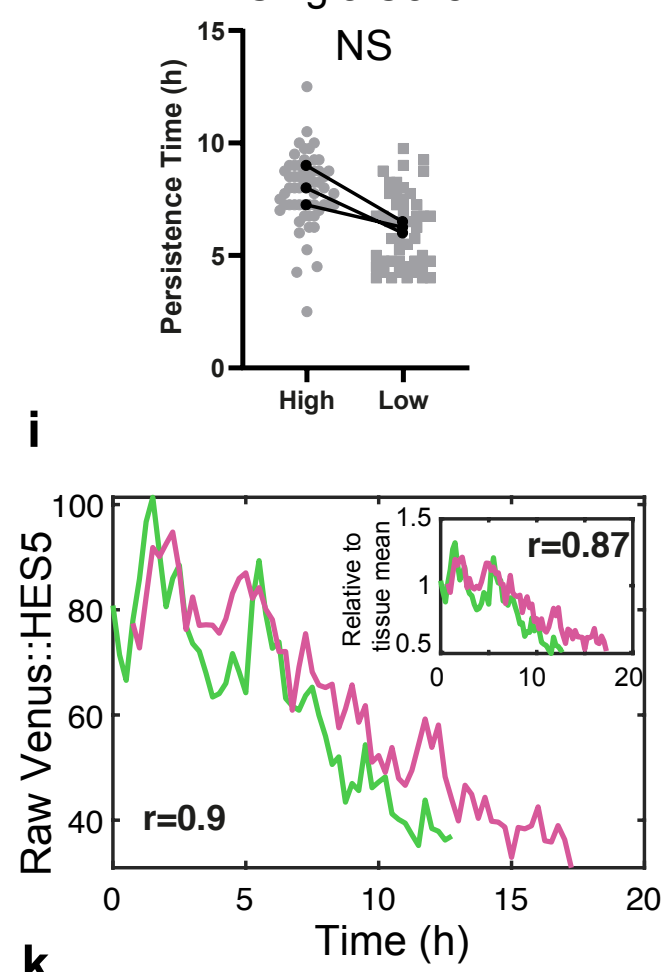

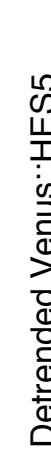

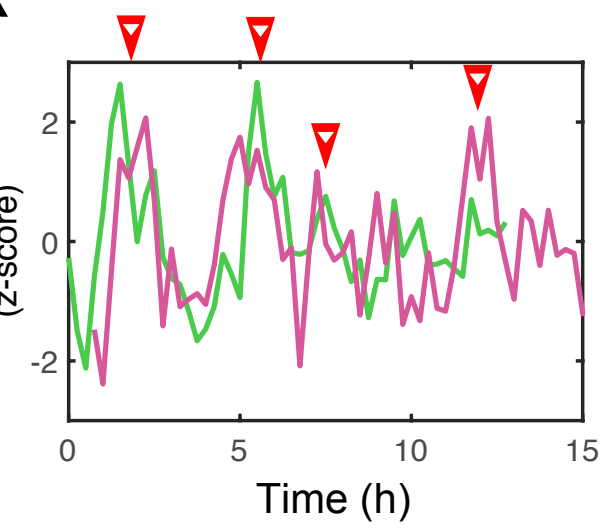

Local Dynamics

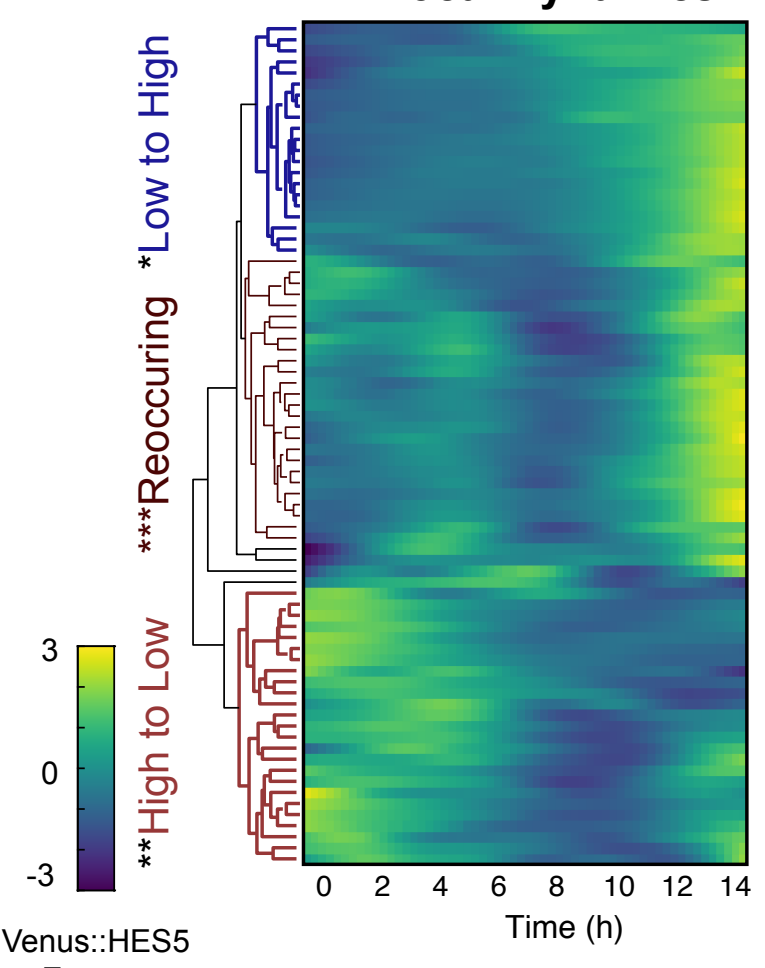

d Local Regions
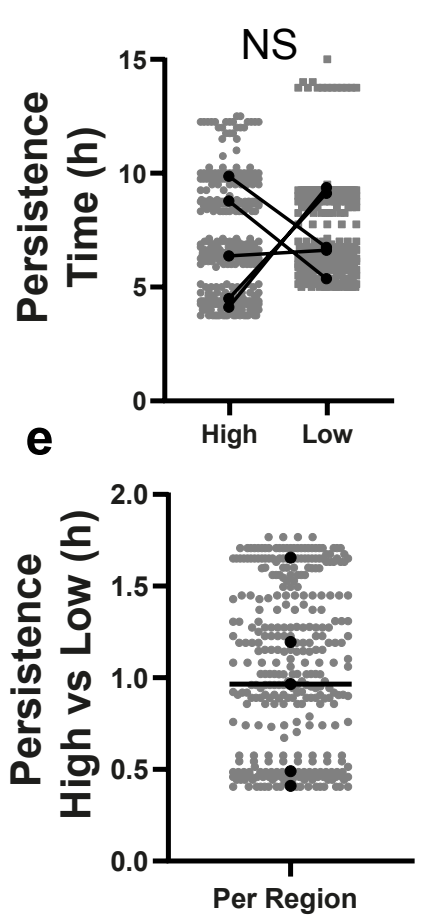

Z-score

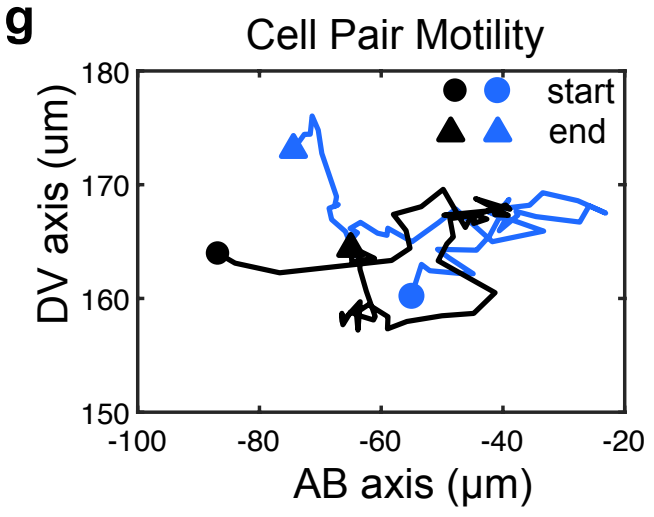

h
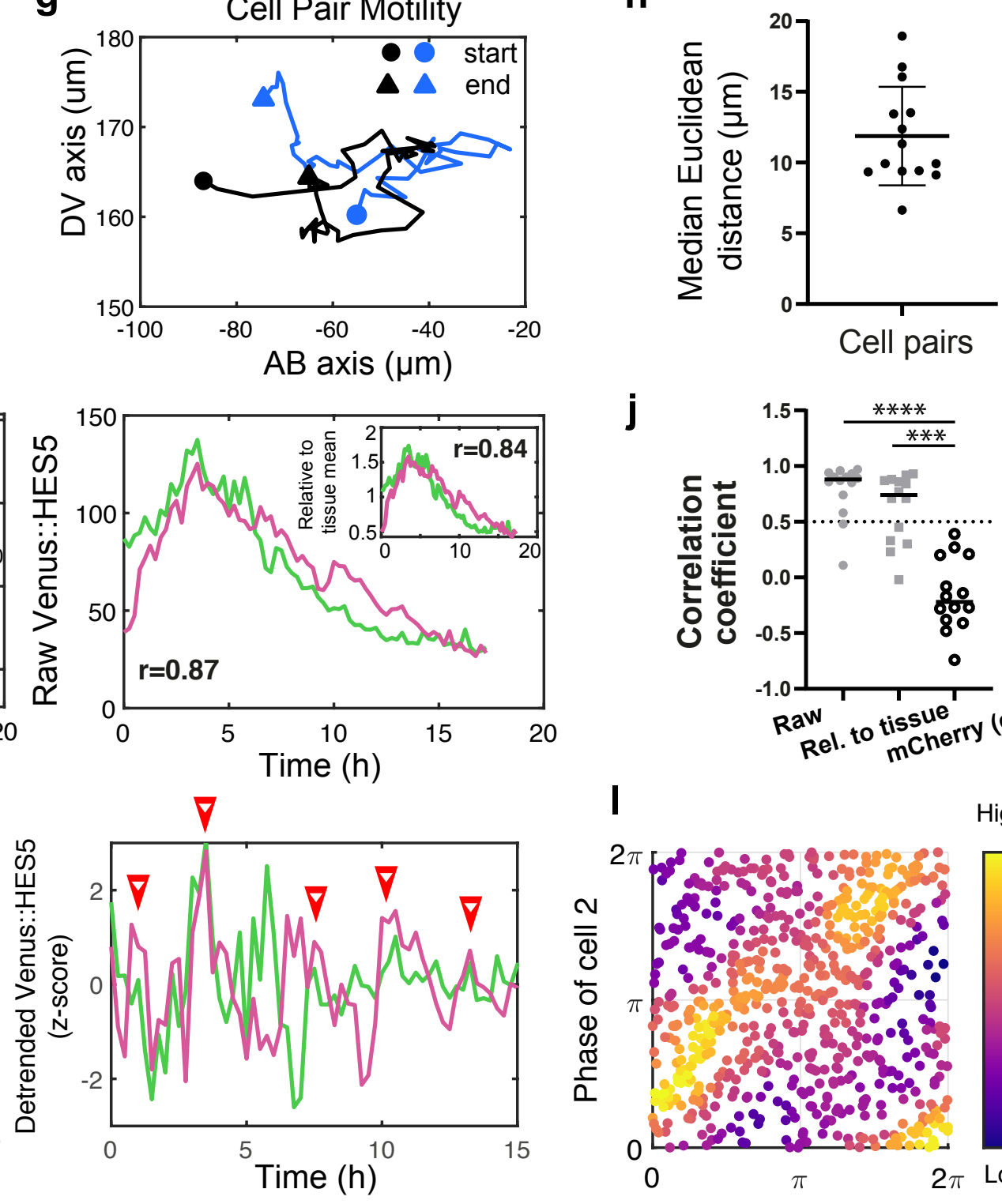

in-phase peaks

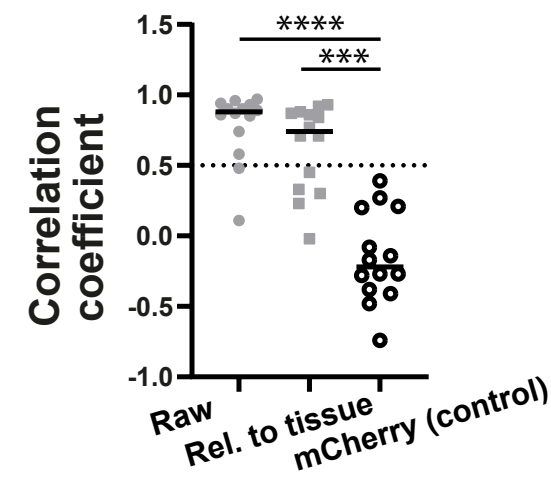

I

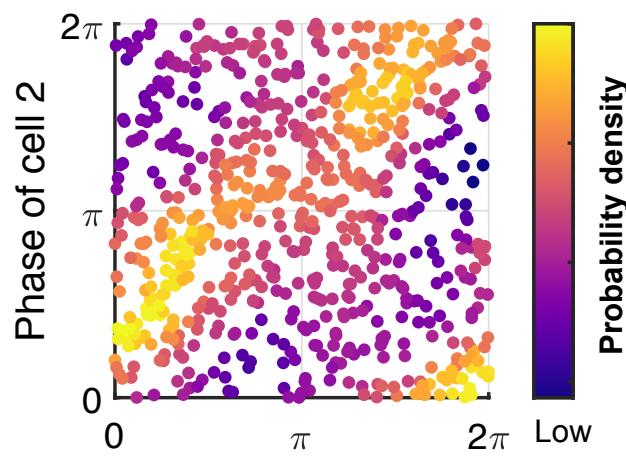

Phase of cell 1 
Figure 4. Notch inhibition increases HES5 pattern persistence.

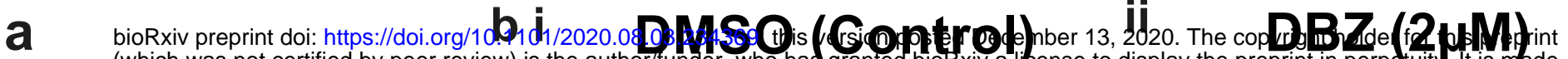
(which was not certified by peer review) is the author/funder, who has granted biokxiv a license to display the preprint in perpetuity. It is made

$* * * *$

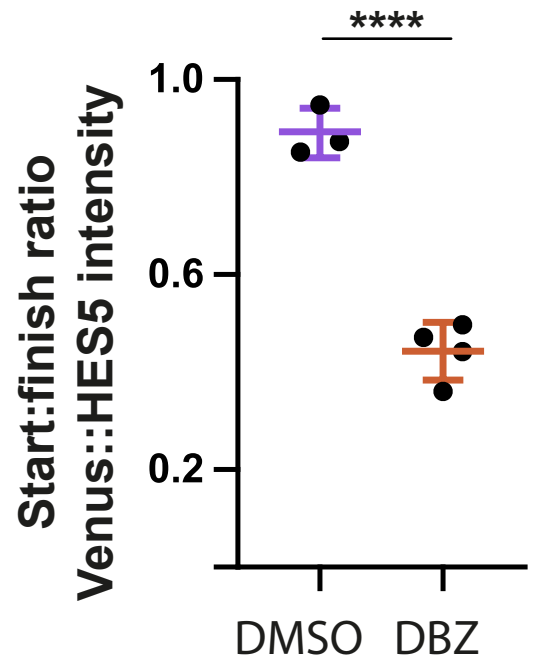

C i

Phase

reconstruction

d

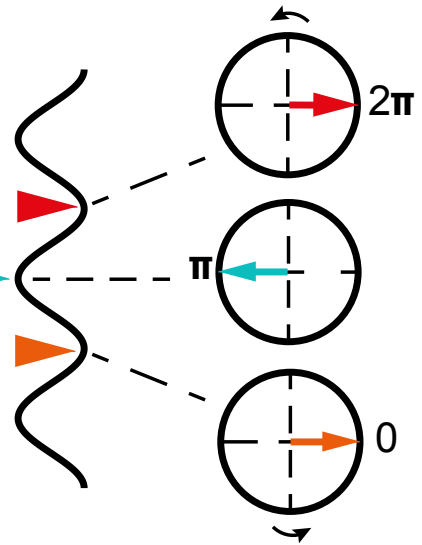

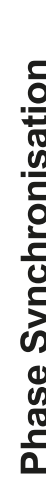

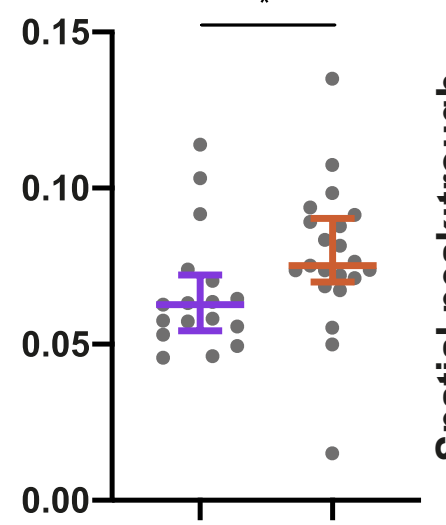

DMSO DBZ
$2 \mathrm{~h} 4 \mathrm{~h} 6 \mathrm{~h} 8 \mathrm{~h} 10 \mathrm{~h} \mathrm{12h}$

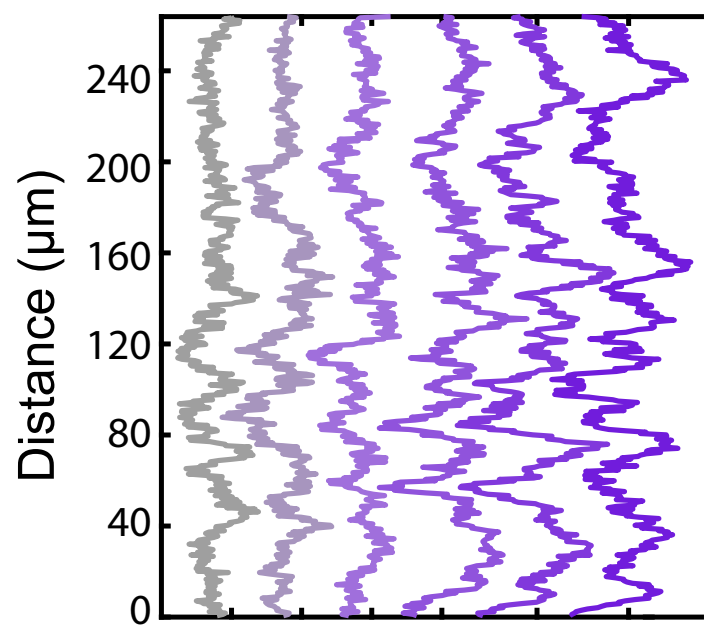

Detrended Venus::HES5 High $\longleftrightarrow$ Low

ii
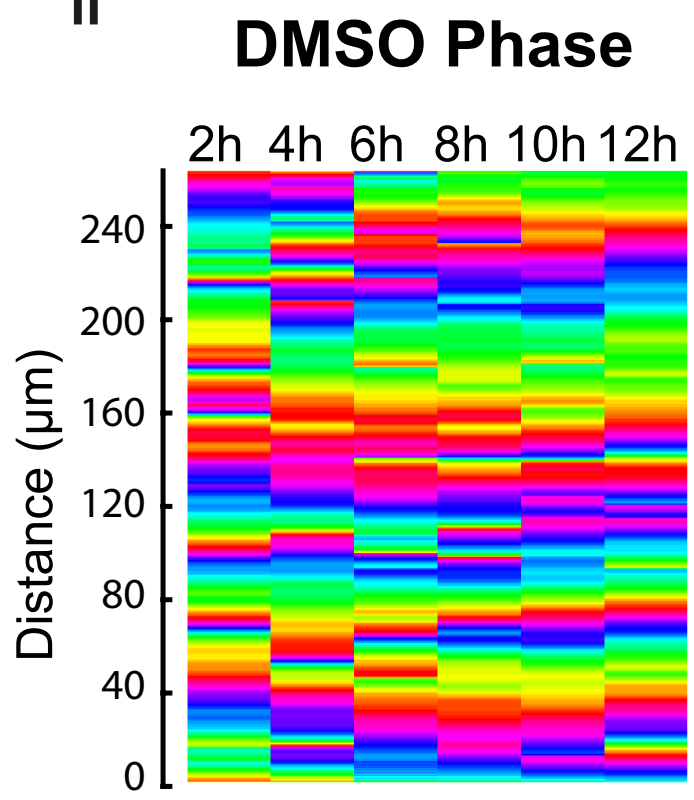

Phase angle

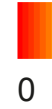

e

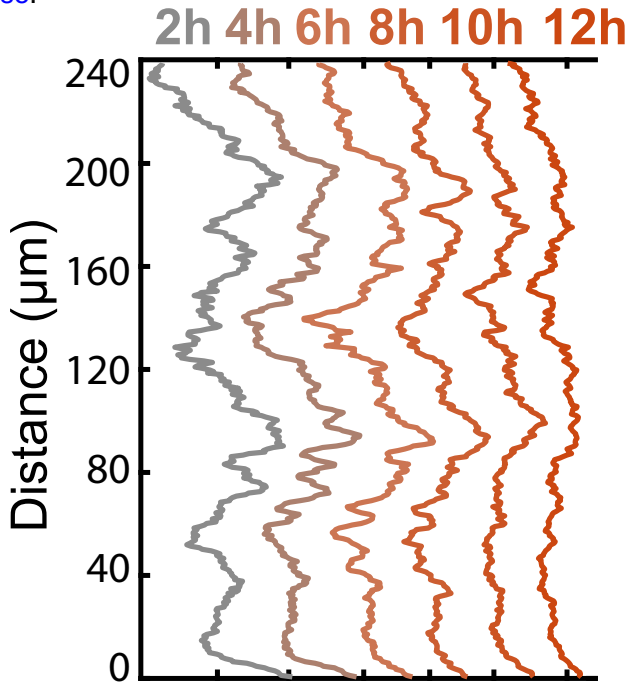

Detrended Venus::HES5

High $\longleftrightarrow$ Low

iii

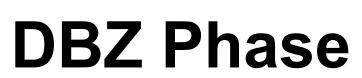

$2 \mathrm{~h} 4 \mathrm{~h}$ 6h $8 \mathrm{~h} 10 \mathrm{~h} 12 \mathrm{~h}$

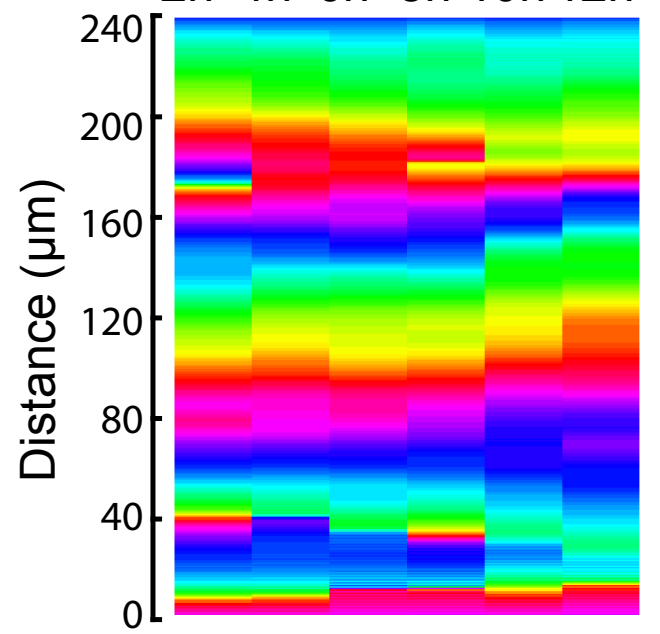

Phase angle

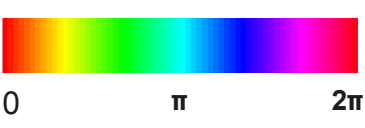

g
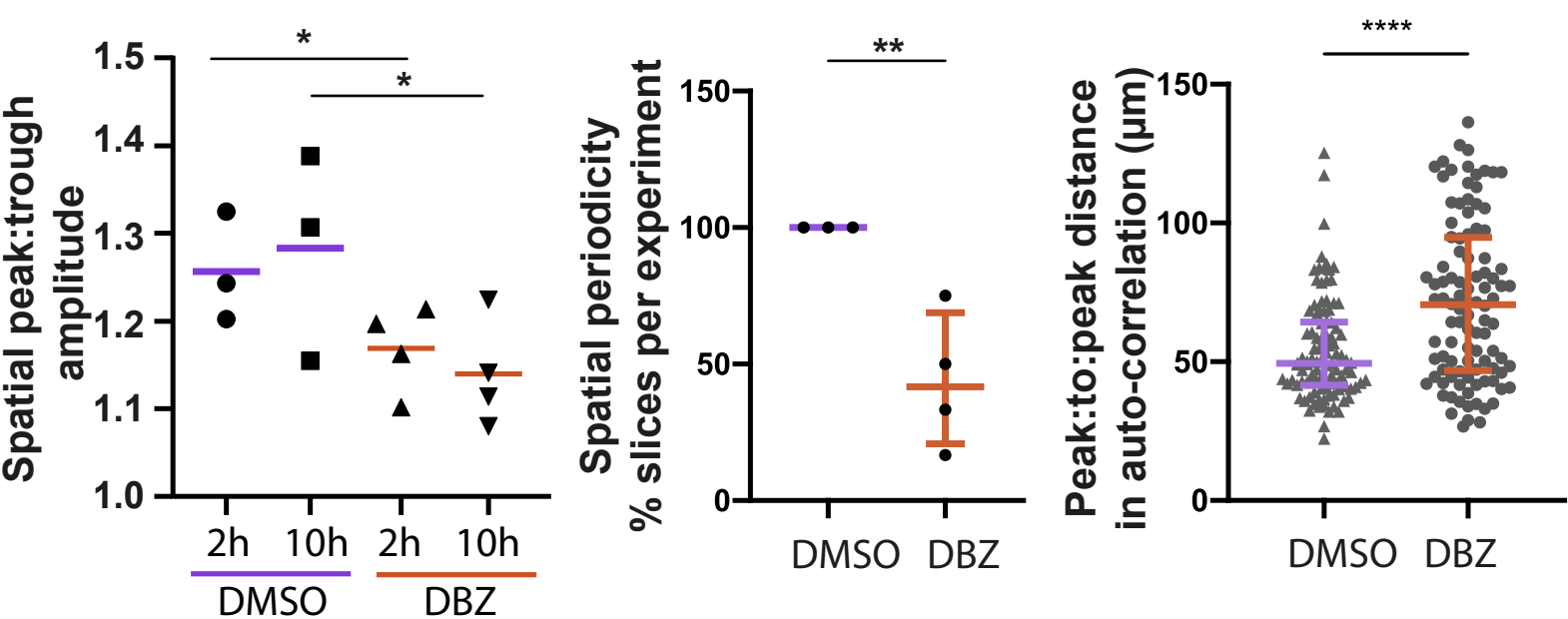

DMSO DBZ 


\section{Figure 5. Mathematical model of cell-cell interactions}

explains the emergence of microclusters. available under aCC-BY-NC-ND 4.0 International license.

a

Inter-cellular Notch-Delta Repression

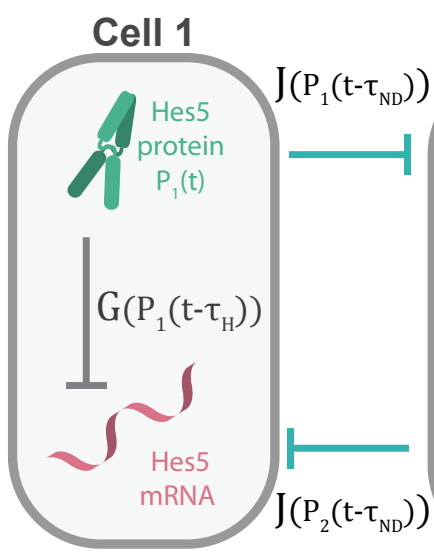

G - Hill function (Hes auto-repression) $\mathrm{J}$ - Hill function (Notch-Delta)

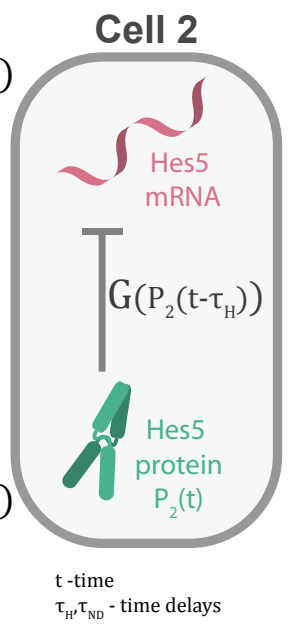

b

Inter-cellular Hill function with delay $\mathrm{J}\left(\mathrm{P}\left(\mathrm{t}-\tau_{\mathrm{ND}}\right)\right)=\frac{1}{1+\left(\frac{\mathrm{P}\left(\mathrm{t}-\tau_{\mathrm{ND}}\right)}{\mathrm{P}_{0}}\right)^{\mathrm{n}}}$
Time delay, $\tau_{\mathrm{ND}}$ Repression Threshold, $\mathbf{P}_{0}$ Hill

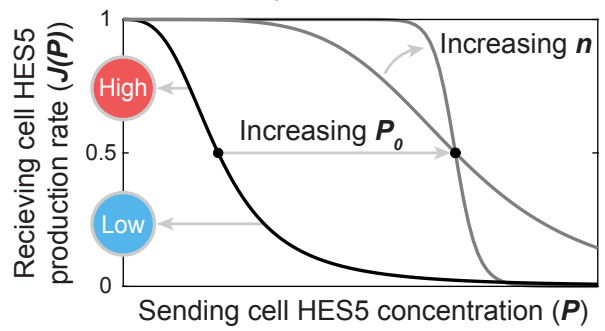

C Multi-cellular
Coupled Model

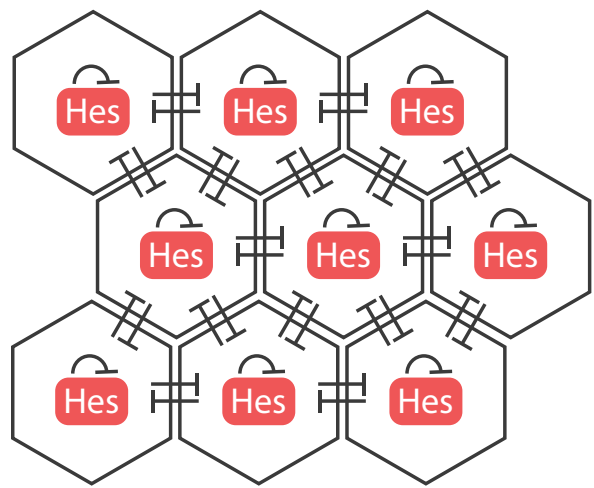

d

Temporal period $(\mathrm{h})$

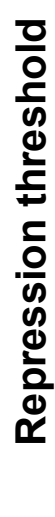

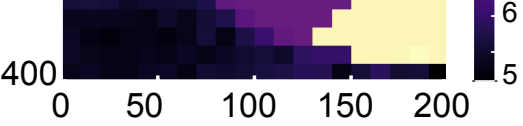

Time delay $(\mathrm{min})$

Coupling Strength

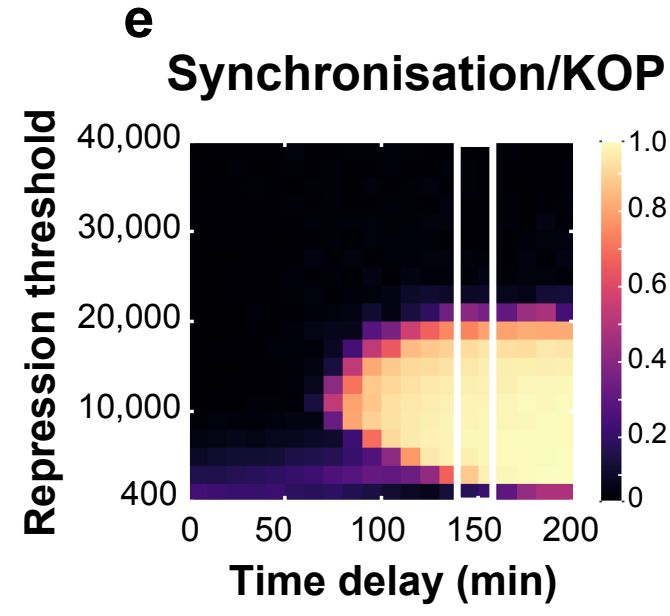

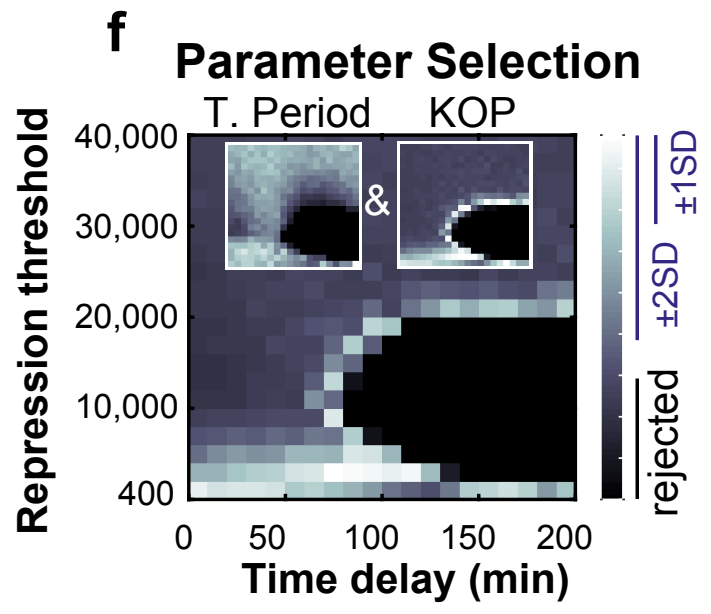

h
Repression Threshold

$$
\text { i }
$$

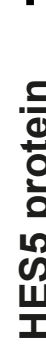

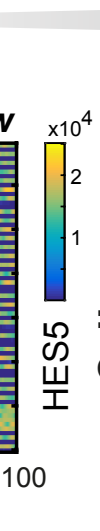

Time (h)

Alternating high-low
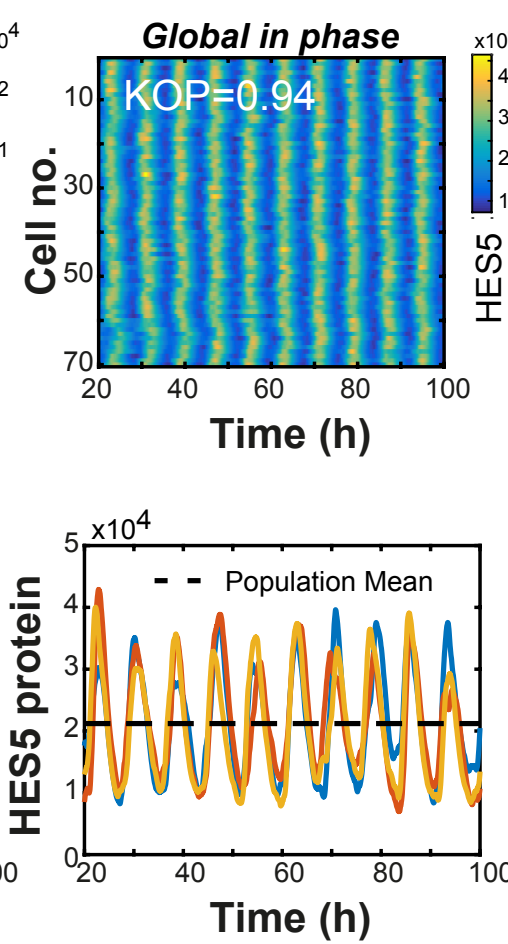

$\checkmark$ microclusters
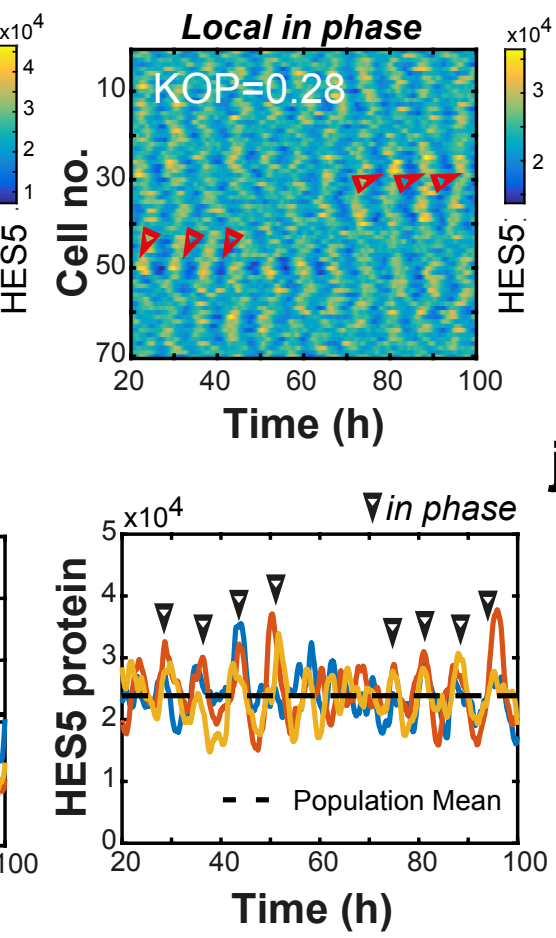

No coupling

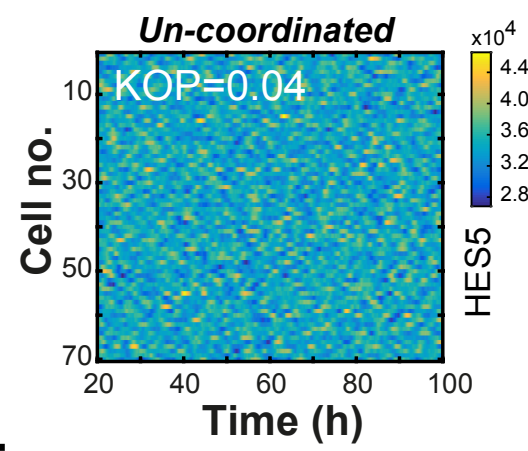

J

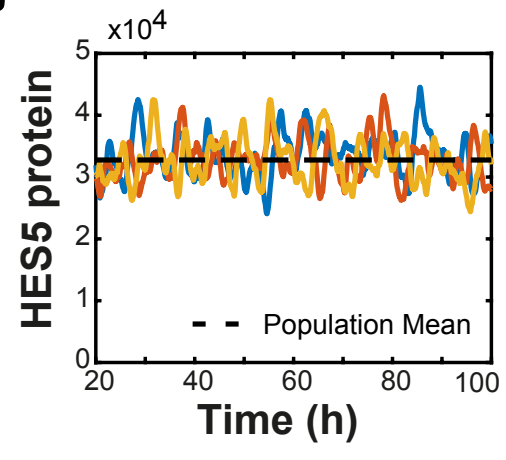




\section{Figure 6. Cell-cell coupling strength can regulate probability of differentiationin multi-celldmodel

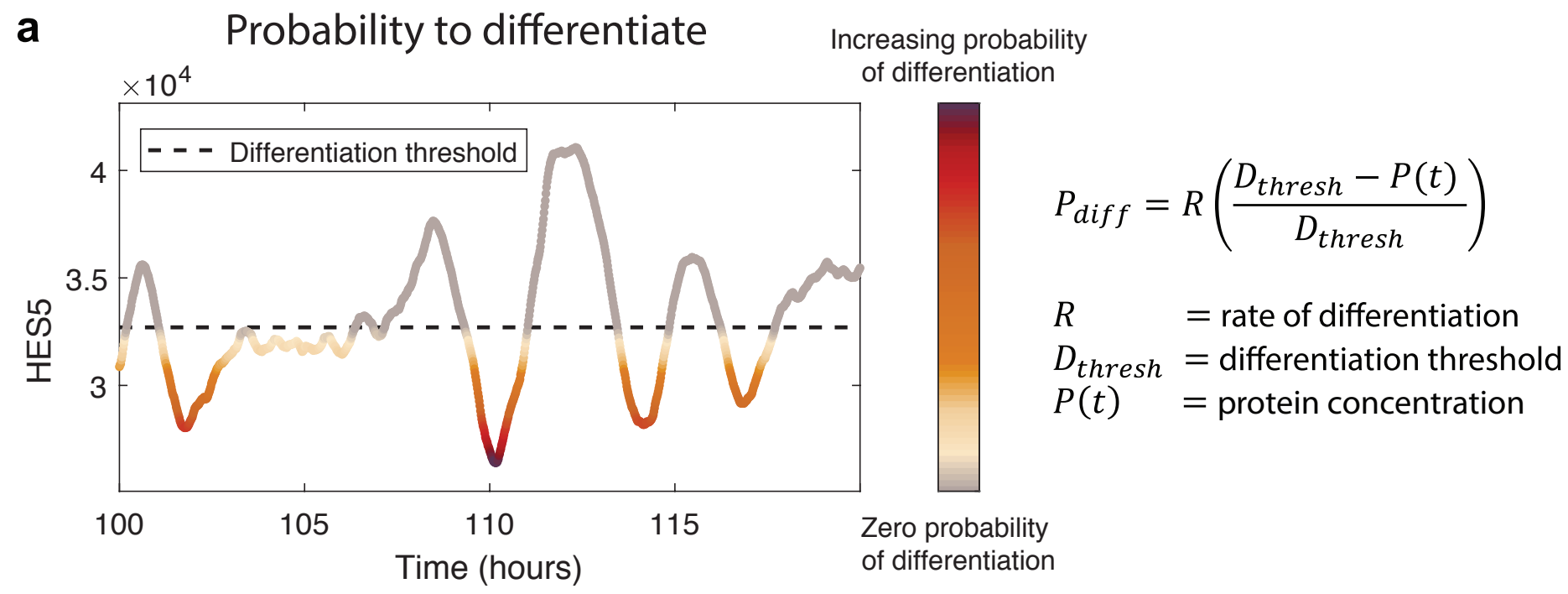

b

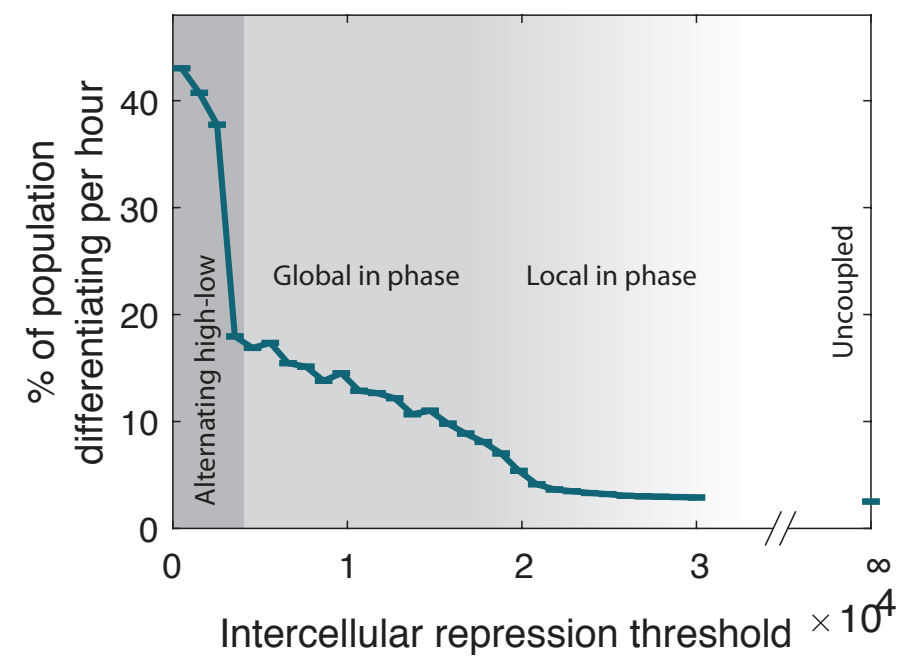

Coupling strength c Coefficient of variation

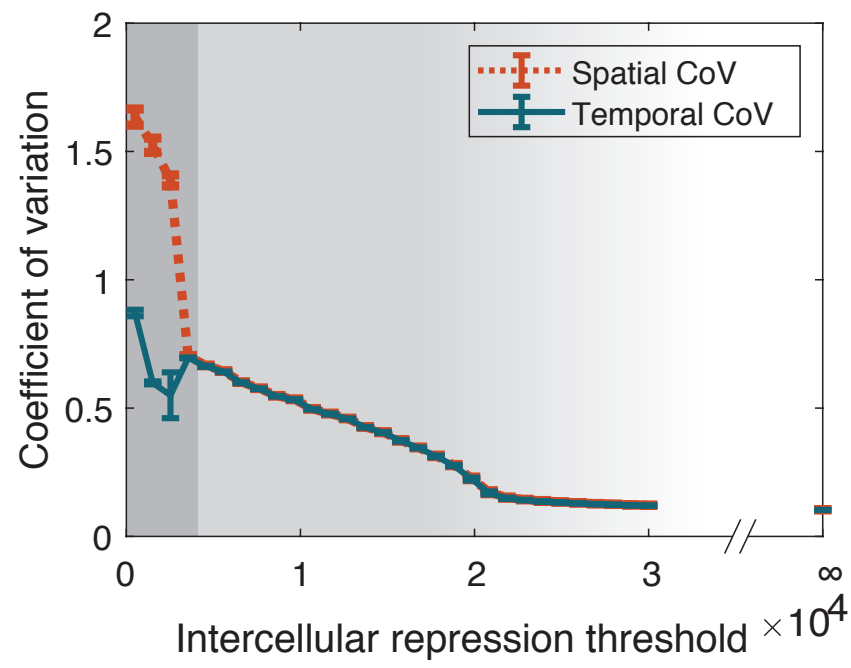

Coupling strength 
Figure 7. Type of HES5 spatial pattern and

\section{coupling strength correlates with rate of}

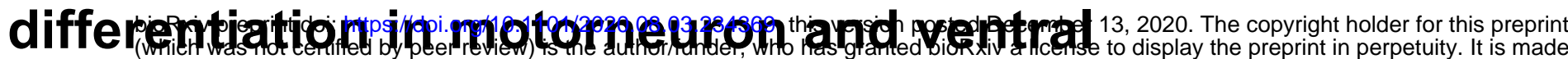
interneuron domains

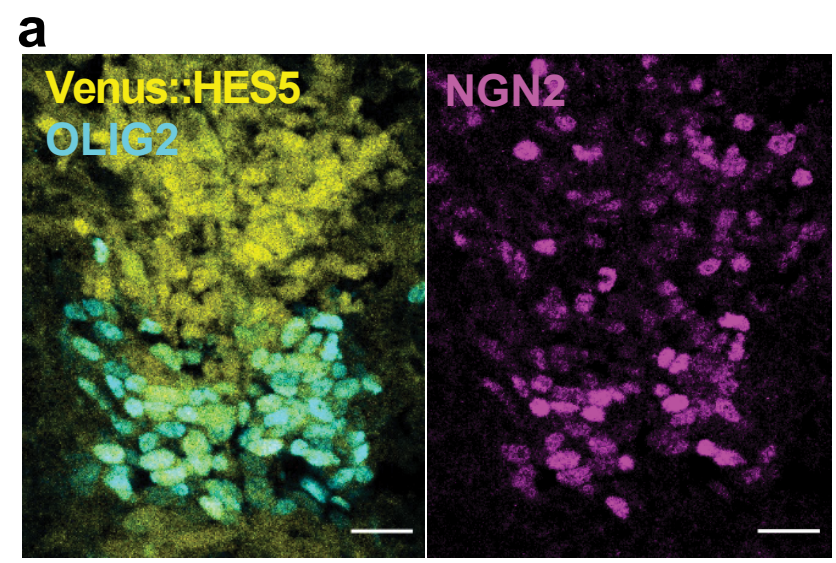

b

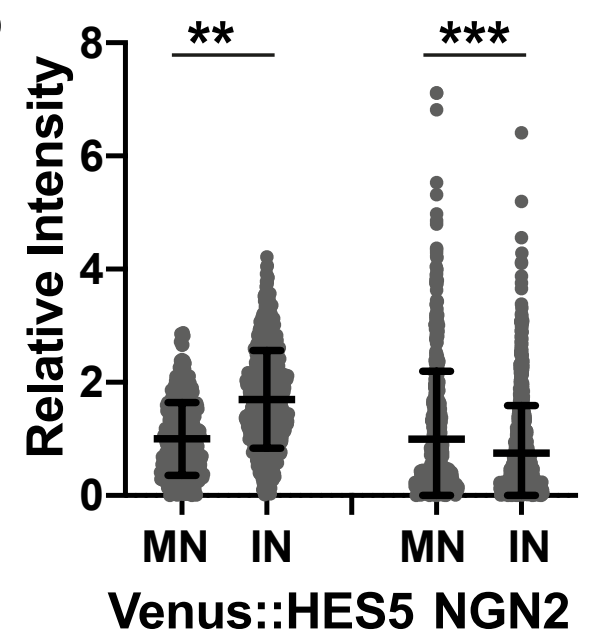

C
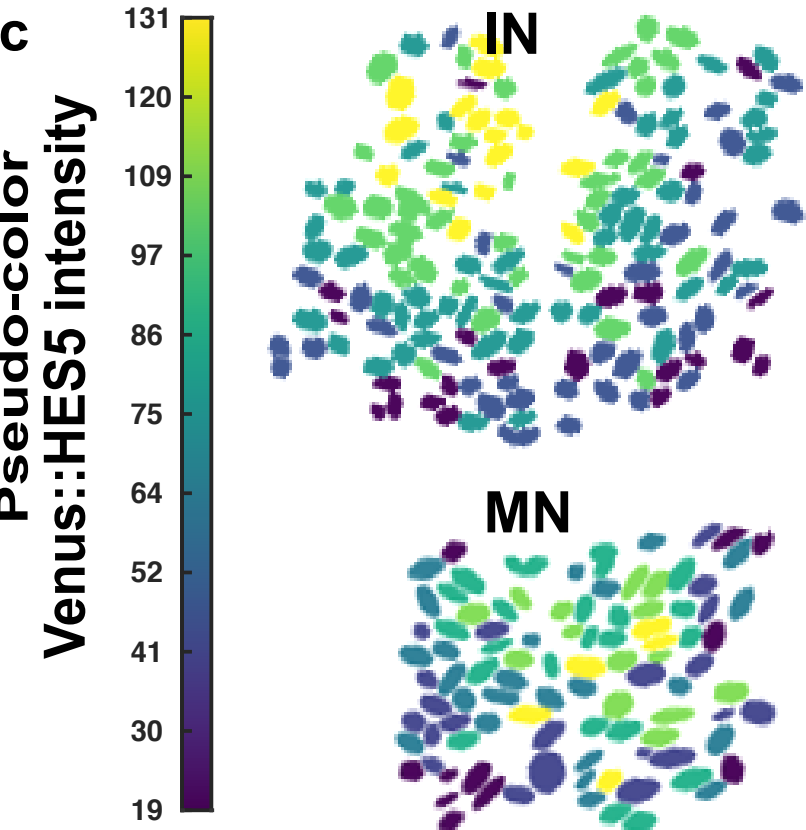

d
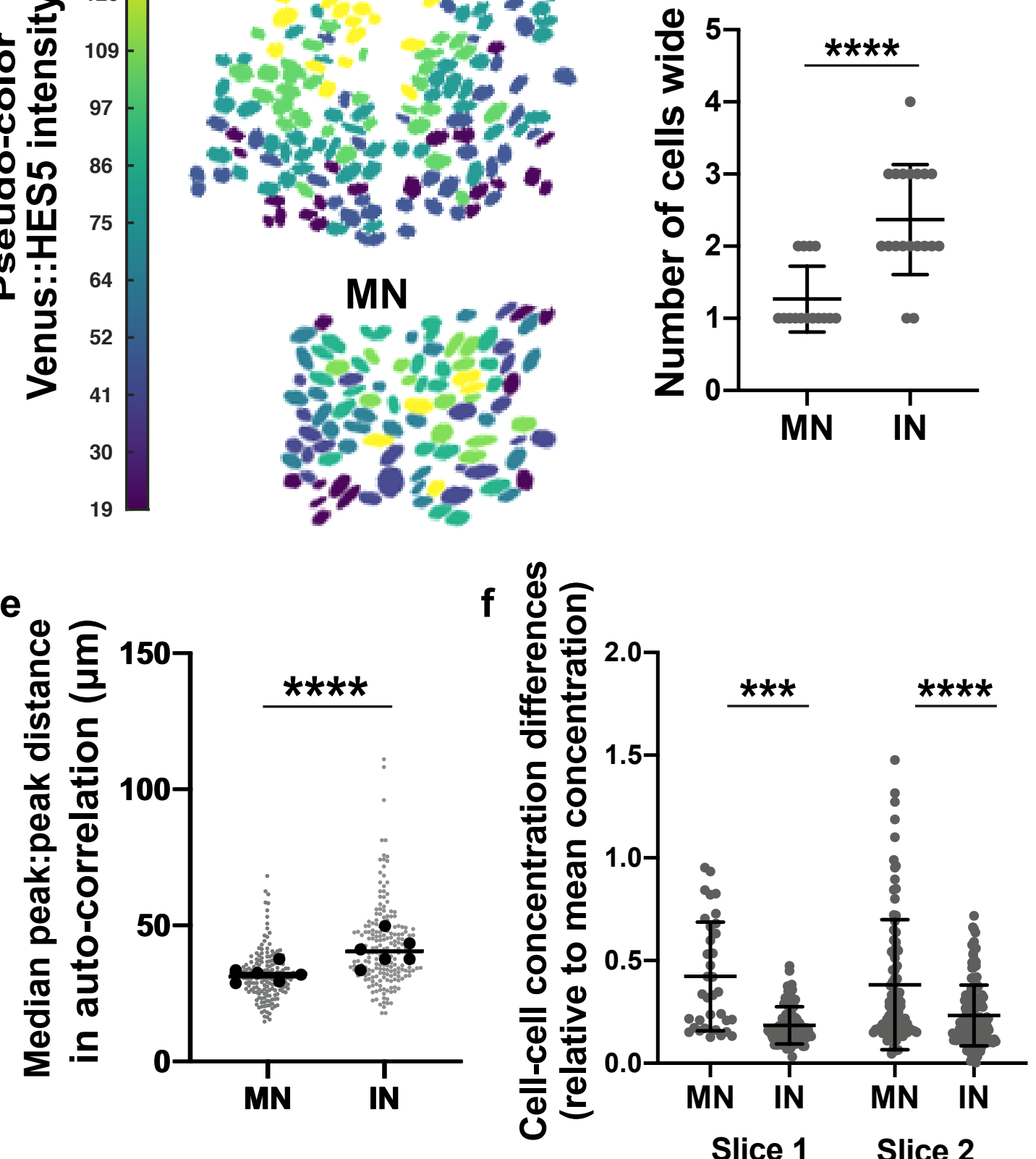
Figure 8. NGN2 expression is spatially periodic and coordinates with the HES5 pattern

a

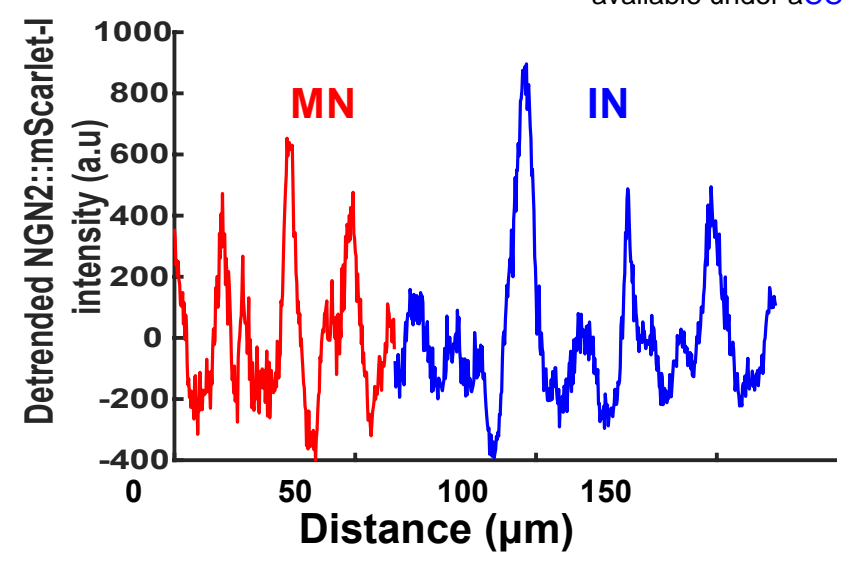

C

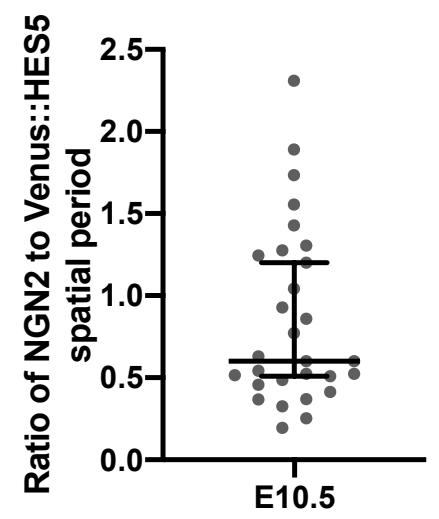

d

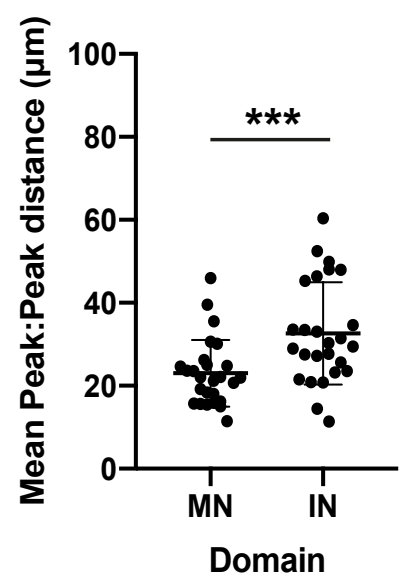

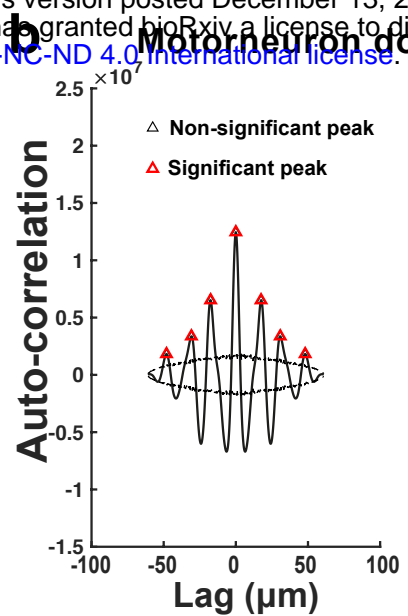

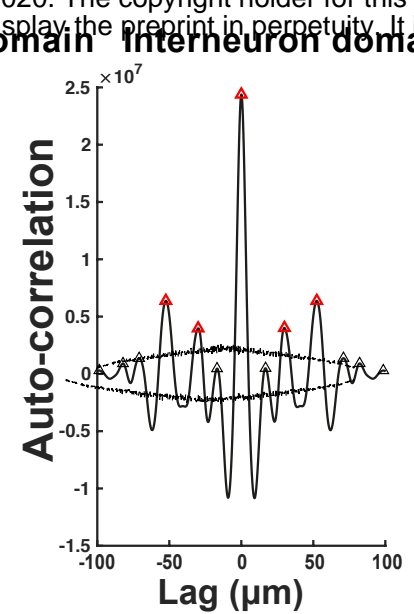

e

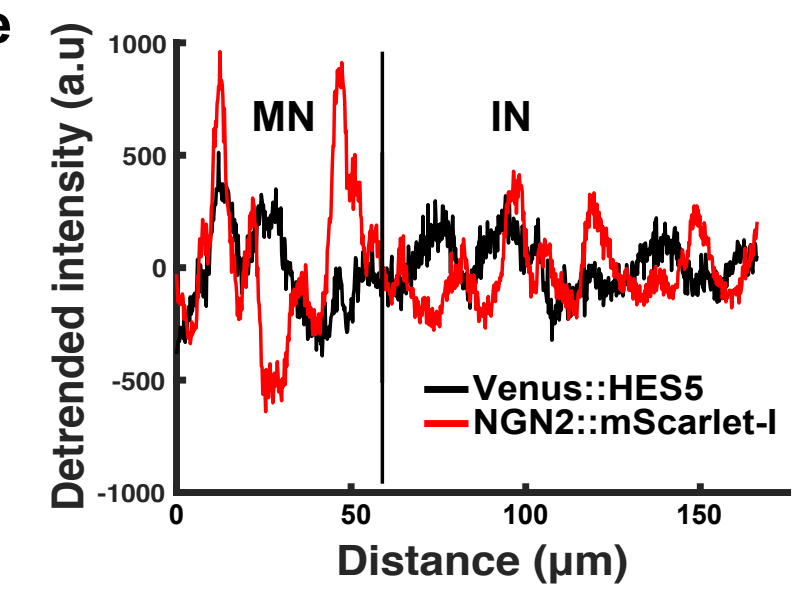

$n^{\text {nade }}$

\author{
.
}

f

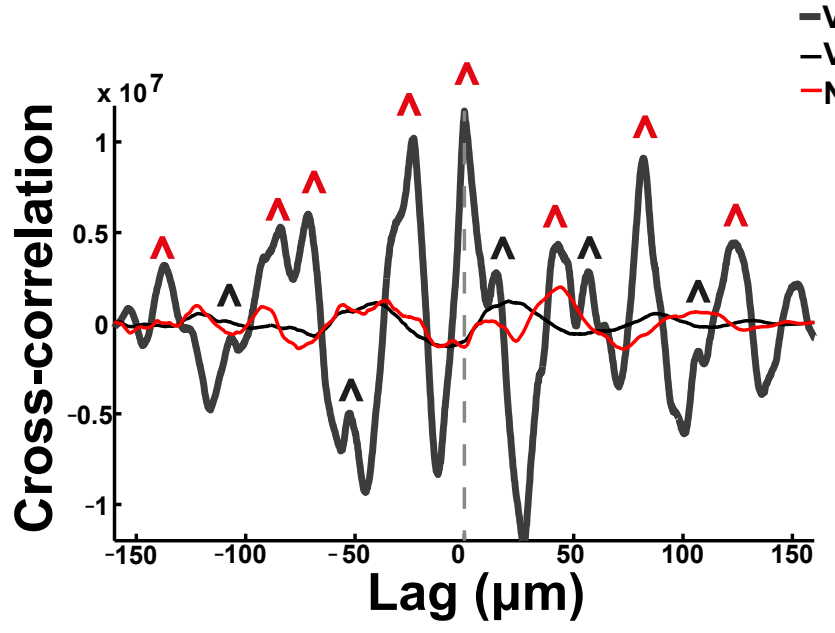

-Venus::HES5 vs NGN2

-Venus::HES5 vs Brightfield

-NGN2 vs Brightfield

^ positively correlated

^ negatively correlated

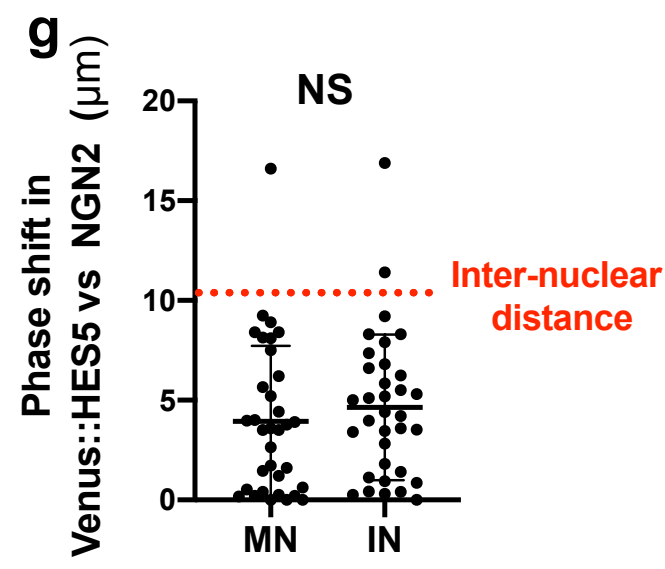

h 要
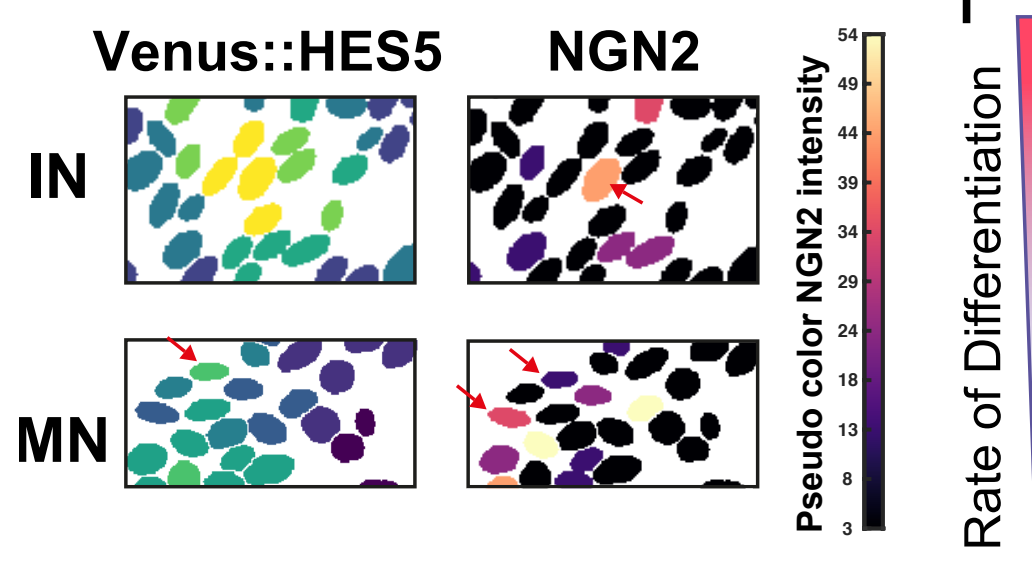

Motorneuron

domain

Alternating
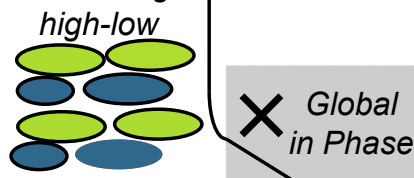

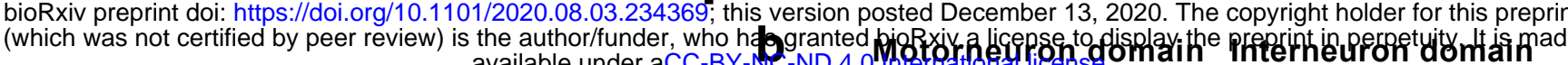

